\title{
Risk Assessment of
}

\section{Necrotizing Enterocolitis in NICU}

\author{
by
}

Irena Zamboni

\begin{abstract}
A thesis submitted to
The Faculty of Graduate Studies and Research in partial fulfillment of the requirements for the degree of

Master of Applied Science in Electrical Engineering
\end{abstract}

Ottawa-Carleton Institute for Electrical and Computer Engineering

Department of Systems and Computer Engineering

Carleton University

Ottawa, Ontario, Canada

May 2008

(C) 2008, Irena Zamboni 


$\begin{array}{ll}\begin{array}{l}\text { Library and } \\ \text { Archives Canada }\end{array} & \begin{array}{l}\text { Bibliothèque et } \\ \text { Archives Canada }\end{array} \\ \begin{array}{l}\text { Published Heritage } \\ \text { Branch }\end{array} & \begin{array}{l}\text { Direction du } \\ \text { Patrimoine de l'édition }\end{array} \\ \begin{array}{l}\text { 395 Wellington Street } \\ \text { Ottawa ON K1A 0N4 } \\ \text { Canada }\end{array} & \begin{array}{l}\text { 395, rue Wellington } \\ \text { Ottawa ON K1A 0N4 } \\ \text { Canada }\end{array}\end{array}$

Your file Votre référence ISBN: 978-0-494-40648-9 Our file Notre référence ISBN: 978-0-494-40648-9

NOTICE:

The author has granted a nonexclusive license allowing Library and Archives Canada to reproduce, publish, archive, preserve, conserve, communicate to the public by telecommunication or on the Internet, loan, distribute and sell theses worldwide, for commercial or noncommercial purposes, in microform, paper, electronic and/or any other formats.

The author retains copyright ownership and moral rights in this thesis. Neither the thesis nor substantial extracts from it may be printed or otherwise reproduced without the author's permission.
AVIS:

L'auteur a accordé une licence non exclusive permettant à la Bibliothèque et Archives Canada de reproduire, publier, archiver, sauvegarder, conserver, transmettre au public par télécommunication ou par l'Internet, prêter, distribuer et vendre des thèses partout dans le monde, à des fins commerciales ou autres, sur support microforme, papier, électronique et/ou autres formats.

L'auteur conserve la propriété du droit d'auteur et des droits moraux qui protège cette thèse. $\mathrm{Ni}$ la thèse ni des extraits substantiels de celle-ci ne doivent être imprimés ou autrement reproduits sans son autorisation.
In compliance with the Canadian Privacy Act some supporting forms may have been removed from this thesis.

While these forms may be included in the document page count, their removal does not represent any loss of content from the thesis.
Conformément à la loi canadienne sur la protection de la vie privée, quelques formulaires secondaires ont été enlevés de cette thèse.

Bien que ces formulaires aient inclus dans la pagination, il n'y aura aucun contenu manquant.

\section{Canada}




\section{Abstract}

Increase in infant survival rates has resulted in an increase of numerous medical complications, such as necrotizing enterocolitis (NEC): "acute, self-limiting, inflammatory bowel disease of premature infants of unclear pathogenesis" (Di Lorenzo, 2000).

In order to assist the medical professionals in diagnosis of NEC, this research focuses on three populations of interest to NEC: total neonatal population, very-lowbirth-weight and gestation age less than 33 weeks. To obtain the minimum data sets for NEC, the performance of two classifiers was assessed: MIRG's ANN RFW tool as well as the LIBSVM tool.

The minimum data sets found for the three NEC models were all unique. The ANN RFW performance was superior for NEC modeling in comparison to the SVM tool, which had performance issues when classifying minority cases. This suggests that data resampling should be considered for other data mining tools as it increases classifier performance. 


\section{Acknowledgements}

To my supervisor, Dr. Monique Frize:

Thank you for encouraging me to pursue my interests in biomedical engineering, pushing me to my limits, and teaching me a lesson in perseverance.

To Jeff, Christophe and the rest of my MIRG fellows:

Thank you for your encouragement, wisdom and support.

To my girls - Daphné \& Shauna:

Thank you beyond words! Hourly, daily, weekly - both of you helped in getting me here and I am simply speechless!

'hip hip'...

\section{To my parents and brother:}

Thank you for your ongoing support and constant motivation throughout my academic career, especially during the dash to the finish line. I couldn't have done it without you! Thank you for helping me succeed...

"We are drowning in information but starved for knowledge."

$\sim$ John Naisbitt 


\section{Contents}

1 INTRODUCTION

1.1 Motivation and Significance of Research ...................................... 1

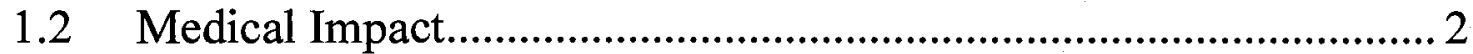

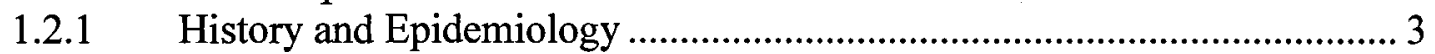

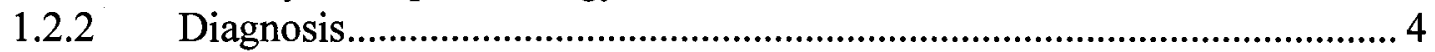

1.2.3 Management and Prognosis .................................................................. 6

1.2.4 Populations of interest for NEC .......................................................... 6

1.2.4.1 NEC in full-term or near-term infants ............................................ 7

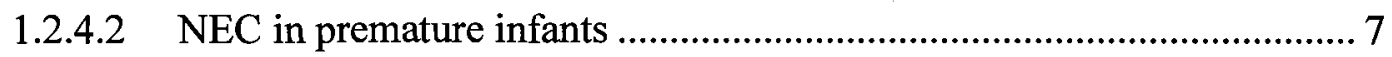

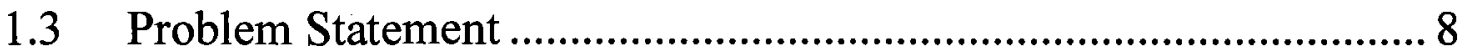

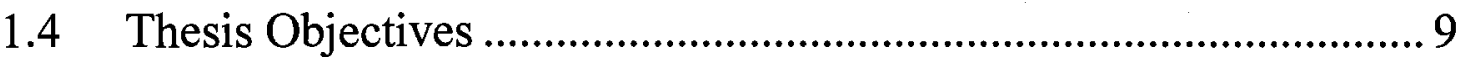

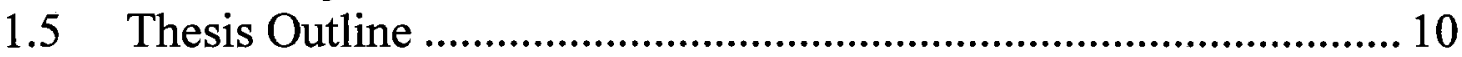

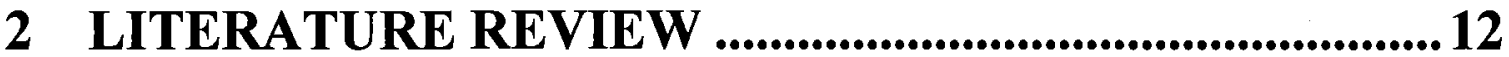

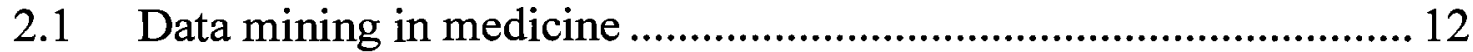

2.2 Neonatal (Severity of Illness) Scoring Systems............................. 15

2.2.1 The Apgar Score ................................................................................ 16

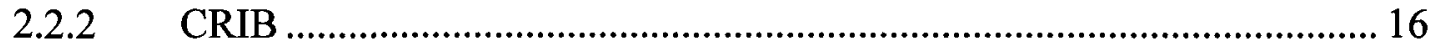

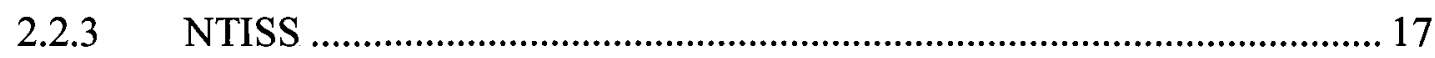

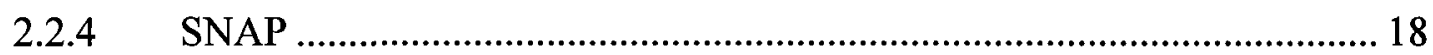

2.2.5 NEC Scoring Systems ............................................................................ 18

2.2.5.1 Presurgical NEC-mortality scoring system.......................................... 19

2.2.5.2 Mathematical model of inflammation in NEC.................................... 22

2.3 Clinical Decision Support Systems ............................................... 23

2.3.1 Model Evaluation Techniques ............................................................. 23

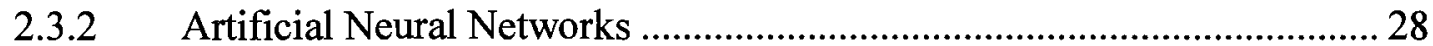

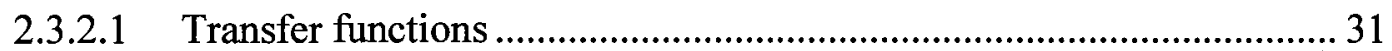

2.3.2.2 ANN RFW tool \& driving parameters................................................. 32

2.3.2.3 Network structure..................................................................................... 35

2.3.3 Support Vector Machines ........................................................................ 37

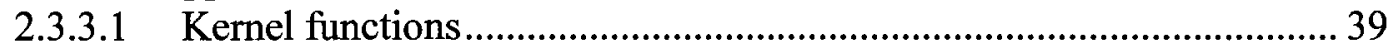

2.3.3.2 Soft Margin Support Vector Classifier: C-SVC .................................... 41

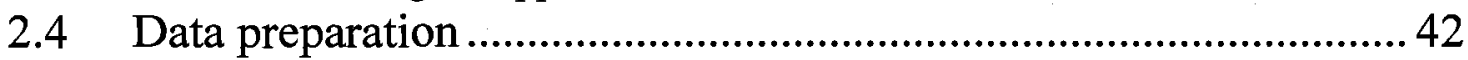

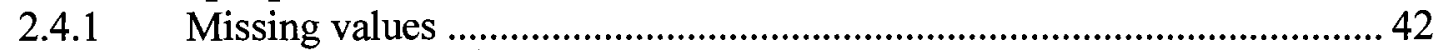

2.4.1.1 CBR uniform weight imputation ...................................................... 44

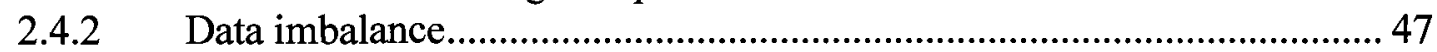

2.4.3 Data dimensionality reduction (DDR) .................................................... 48

2.4.3.1 Relative Weight Reduction Method (ANN) ............................................ 49 


\section{METHODOLOGY}

3.1 Data Understanding and Preparation ..........................................52

3.1.1 ANN RFW vs. C-SVC datasets for outcome prediction.............................54

3.2 Finding the Minimum Data Set.................................................5

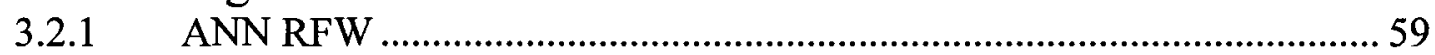

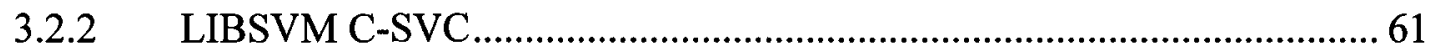

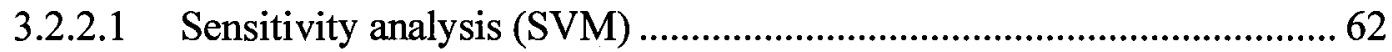

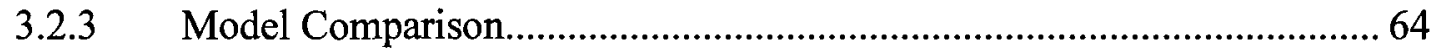

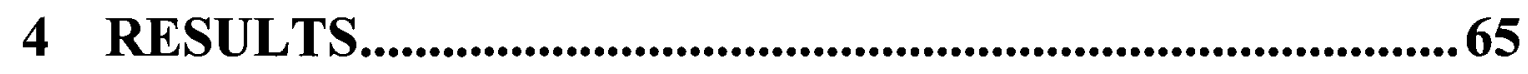

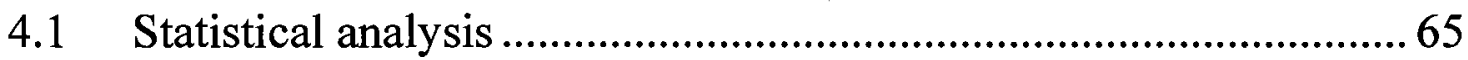

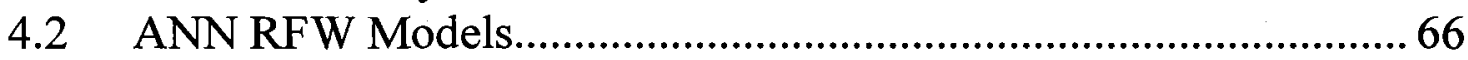

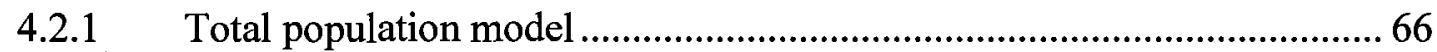

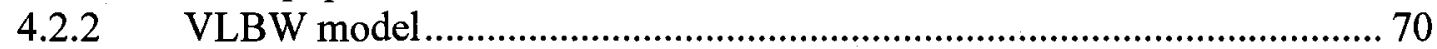

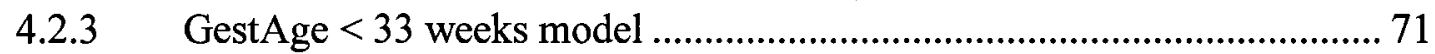

4.3 LIBSVM C-SVC Models............................................................ 73

4.3.1 VLBW model................................................................................... 74

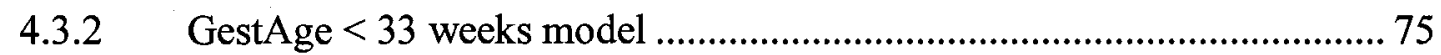

4.3.3 Total population model ........................................................................... 75

4.4 Model Comparison: ANN vs. SVM............................................ 78

4.4.1 Additional ANN \& SVM models ......................................................... 79

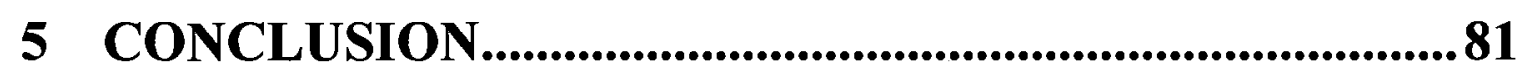

5.1 Summary of Contributions ............................................................. 81

5.2 Future Research.................................................................... 82

REFERENCES ....................................................................85

APPENDIX A: Features of NEC .......................................91

APPENDIX B: Neonatal Scoring Systems .............................94 


\section{List of Tables}

\section{Chapter 2 - Literature Review}

Table 2.1: Research uses of predictive scored in neonatology (Dorling, Field, \&

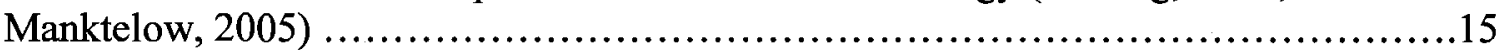

Table 2.2: Statistical summary of Kessler et. al. (2006) study ............................19

Table 2.3: Laboratory values, survival and NEC-score in operated and conservatively treated infants (Kessler et al., 2006) ............................................20

Table 2.4: Laboratory value, BW, GA and NEC-score in survivors and non-survivors

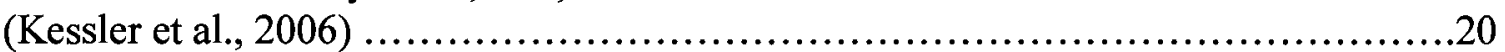

Table 2.5: NEC-score (Kessler et al., 2006) ............................................21

Table 2.6: $2 \times 2$ confusion matrix for a 2-class classification system ..................24

Table 2.7: Common SVM kernel functions ......................................40

Table 2.8: CNN database missing value statistics (Townsend, 2007) ...................45

Table 2.9: Distribution of missing values in the

$13584 \mathrm{CNN}$ database (Townsend, 2007)

\section{Chapter 3 - Methodology}

Table 3.1: Day 1 variables used in NEC modeling ................................52

Table 3.2: ANN RFW data distribution - NEC model for the total population ...........56

Table 3.3: LIBSVM C-SVC data distribution - NEC model for the total population .....56

Table 3.4: ANN RFW data distribution - NEC model for the VLBW population ........57

Table 3.5: LIBSVM C-SVC data distribution - NEC model for the VLBW population .58

Table 3.6: ANN RFW data distribution - NEC model for gestation age $<33$ weeks ......58

Table 3.7: LIBSVM C-SVC data distribution - NEC model for gestation age $<33$ wks ...58

\section{Chapter 4 - Results}

Table 4.1: Spearman correlation and 2-tailed statistical significance of input variables with respect to the outcome of NEC

Table 4.2: ANN RFW results for Model A*

Table 4.3: Variables and weights for best performance structures for Model $A^{*} \ldots \ldots .667$

Table 4.4: ANN RFW results for Model B* .......................................68

Table 4.5: Variables and weights for best performance structures for Model $\mathrm{B}^{*}$.......69

Table 4.6: ANN RFW results for the VLBW model .................................70

Table 4.7: Variables and weights for best performance structures of the VLBW model .70

Table 4.8: ANN RFW results for the GestAge $<33$ weeks population.................72

Table 4.9: Variables and weights for best performance structures of the GestAge $<33$ weeks model ........................................................................ 72

Table 4.10: Grid search for the $\mathrm{C}$ and $\gamma$ parameters for VLBW ........................74

Table 4.11: Grid search for the $C$ and $\gamma$ parameters for GestAge $<33$ weeks ..............75

Table 4.12: Grid search for the $\mathrm{C}$ and $\gamma$ parameters for the total population .............76

Table 4.13: LIBSVM C-SVC results for the total population model .....................76

Table 4.14: LIBSVM C-SVC results for the total population model ...................77

Table 4.15: SNAPPE-II performance of the ANN RFW and LIBSVM C-SVC tools ....80

Table 4.16: Occurrence of SNAPPE-II variables in minimum datasets ................ 80 


\section{List of Figures}

\section{Chapter 2 - Literature Review}

Figure 2.1: The six-step DMKD process model (Cios \& Moore, 2002) ................13

Figure 2.2: An ROC graph (Fawcett, 2006) ...........................................26

Figure 2.3: Information processing unit - the neuron (Pan, 2002) .......................29

Figure 2.4: (a) 2-layer network; (b) 3-layer network ................................ 30

Figure 2.5: Hyperbolic transfer function ........................................ 32

Figure 2.6: The optimal hyperplane for a linear 2-class classifier, represented by circles and diamonds (Chen, Lin, \& Scholkopf, 2005) ........................................ 


\section{List of Appendices}

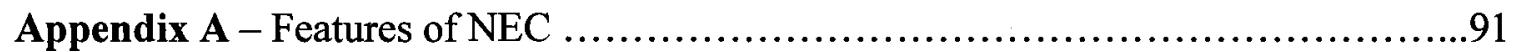

Table A-1: Clinical Characteristics and Laboratory \& Radiographic Features of NEC (McAlmon, 2004) ............................................................91

Table A-2: Modified Bell Staging Criteria (Bell et al., 1978), (Panigrahi, 2006)........992 Figure A-1: (a) Supine and (b) cross-table lateral X-ray images of a NEC patient's abdomen (Epelman et al., 2007).

Appendix B - Neonatal Scoring Systems 94

Table B-1: The Apgar Score (Beachy \& Deacon, 1993)

Table B-2: CRIB (The International Neonatal Network, 1993) ........................94

Table B-3: CRIB II (Parry, Tucker, \& Tarnow-Mordi, 2003) .......................95

Table B-4: NTISS scoring system (Ennett, 2003)

Table B-5: SNAP scoring system (Richardson, Gray, McCormick, Workman, \&

Goldmann, 1993) 97

Table B-6: SNAPPE-II scoring system (Ennett, 2003) 
Glossary (definitions)

ANN: Artificial Neural Network

ANN RFW: Artificial Neural Network Research Framework (tool developed by MIRG)

APGAR: Appearance, Pulse, Grimace, Activity, and Respiration score

BPD: Bronchopulmonary dysplasia

C-SVC: C Support Vector Classification

CBR: Case-Based Reasoner

CDSS: Clinical Decision Support System

CHEO: Children's Hospital of Eastern Ontario

CNN: Canadian Neonatal Network

CRIB: Clinical Risk Index for Babies

IR: Infrared Imaging

IVH: Intraventricular hemorrhage

k-NN: k-Nearest Neighbour algorithm

LIBSVM: Library for Support Vector Machines

MIRG: Medical Information technologies Research Group

NEC: Necrotizing enterocolitis

NICU: Neonatal Intensive Care Unit

NTISS: Neonatal Therapeutic Intervention Scoring System

RBF: Radial Basis Function

SNAP: Score for Neonatal Acute Physiology

SNAPPE: Score for Neonatal Acute Physiology Perinatal Extension

SVM: Support Vector Machine 


\section{INTRODUCTION}

\subsection{Motivation and Significance of Research}

Motivation and significance of this research stems from two equally important perspectives: medical and technical.

From the medical perspective, data collection from patients admitted to a hospital can aid both medical professionals and administration staff. Medical professionals can use previously collected data to analyze trends in medical conditions. Applying this knowledge to patients exhibiting similar symptoms, healthcare staff can act quickly in providing appropriate medical care. With regards to the hospital administrative staff, analysis of patient data can help the hospital in terms of their resource allocations and budget assignments, including equipment management and staff. This is extremely valuable as different hospitals tailor to different patient types and specialize in particular health care divisions; ex. neonatal health, oncology centres etc. Analyzing yearly trends, and even monthly or daily trends, can help hospital administration determine the number of beds it needs to have, specialty units, other equipment resources, and medical staff.

From the technical perspective, in order to assist medical staff and hospital administration in analyzing trends in data and making decisions in a timely manner, data mining tools have come into play. Improvements in computer technology drive the goal to integrate data mining tools with the Clinical Decision Support Systems (CDSSs) so that they may be of use to medical staff in the near future. Medical Information technology Research Group (MIRG) uses the Artificial Neural Network Research Framework Tool (ANN RFW) as the base of its CDSS. MIRG uses the ANN RFW to 
find trends in medical data and develop prediction models for medical outcomes such as mortality, bronchopulmonary dysplasia (BPD), intraventricular hemorrhage (IVH), length of stay (LOS) and length of ventilation (LOV).

With strong medical and technical perspectives, this thesis looks at an additional medical complication in order to enrich the current MIRG ANN RFW tool: necrotizing enterocolitis (NEC). Understanding the NEC medical problem, as described in section 1.2 , is necessary to fully comprehend the choice of models examined in this thesis.

\subsection{Medical Impact}

Necrotizing enterocolitis, $N E C$, is the most common neonatal gastrointestinal (GI) emergency (Lin \& Stoll, 2006), (Panigrahi, 2006). It is a medical complication that has puzzled healthcare professionals for decades, all the while bolstering its 'enigmatic' reputation due to intriguing and complex interaction of many physiological factors. Since the disease causes portions of the bowel to undergo necrosis, i.e. tissue death, medical literature occasionally refers to it as intestinal or bowel gangrene (Gupta, Burke, \& Herson, 1994). Even more alarming is the fact that although medical and surgical techniques are making great advances, in the last few decades mortality and morbidity due to NEC remain high or have been increasing (Panigrahi, 2006). The increase in NEC over the decades is attributed partially to the increase in the number of low birth babies that are surviving due to significant advances in medical technology. A brief overview of history, epidemiology and pathogenesis, diagnosis, management and prognosis, and the populations of interest for NEC are covered. 


\subsubsection{History and Epidemiology}

The earliest accounts of NEC medical literature dates back to 1888, when Paltauf reported clinical and pathological findings of what is considered consistent with NEC today, along with Generisch in 1891 and the report of abdominal distension in a 45-hourold neonate (Panigrahi, 2006). Furthermore, Panigrahi (2006) reports that although NEC was reported in medical literature over the span of the century, it was not accepted as a 'distinct disease entity' until the 1950s, and it was not until the 1ate 1960s and early 1970s that it attracted the attention of surgeons, pathologists and physicians. Medical literature also recognizes the fact that because of the wider recognition of this devastating medical complication, and an increase of preterm birth survivals, NEC has become a serious medical challenge and problem worldwide (Panigrahi, 2006).

Medical experts agree on the fact that NEC has a very complex and multifactorial pathogenesis (McAlmon, 2004), (Kling \& Hutter, 2003). Studies of the disease indicate that it varies from centre to centre and from year to year, and that there are endemic (regional) and epidemic (widespread) occurrences of it (Luig \& Lui, 2005a). It is estimated that anywhere between 0.3 to 2.4 cases occur in every 1,000 live births; more particularly with NEC occurring in 2 to $5 \%$ of all NICU admissions and 5 to $10 \%$ of VLBW infants (McAlmon, 2004). In an attempt to identify risk factors related to NEC, several clinical studies show that two risk factors in particular lead the increased predisposition to development of NEC because of the immaturity of the gastrointestinal tract - prematurity and low birth weight (LBW). As such, gestational age and birth weight are important determinants of mortality risk in NEC (Di Lorenzo, 2000). Characteristics set the mean gestation age of onset at between $30-32$ weeks; 
approximately $10 \%$ of infants are full-term; mean age at onset is 12 days, while mode is 3 days; approximately $90 \%$ of infants have been fed prior to disease onset (McAlmon, 2004). According to McAlmon (2004), infants exposed to cocaine are also up to 2.5 times at an increased risk of developing NEC because "the vasoconstrictive and hemodynamic properties of cocaine may promote intestinal ischemia", resulting in severe intestinal gangrene, perforation and mortality. Even more staggering are mortality statistics of NEC, regardless of medical or surgical interventions: $9-28 \%$; with the mortality of infants weighing less than $1,500 \mathrm{~g}$ being up to $45 \%$ (McAlmon, 2004).

\subsubsection{Diagnosis}

As NEC outcome depends significantly on the time of diagnosis, the earlier that the detection and diagnosis, the better the chance of survival and prognosis. McAlmon's (2004) summary of clinical characteristics as well as laboratory and radiographic features used for NEC diagnosis can be seen in appendix A, table A-1. There is no single NEC-specific test for diagnosis.

Of the various clinical and laboratory features used in diagnosis of NEC, there are also additional medical features that are not NEC specific. These are used in what is known as 'differential diagnosis'. The differential diagnosis characteristics that can be potentially helpful in diagnosing NEC are the presence of sepsis and feeding intolerance. Presence of blood in the stool can also often be an indication of an ulcer or other medical problems, not just NEC.

However, the most important diagnostic tool is the imaging modality abdominal radiography (X-ray) (Epelman et al., 2007). Abdominal radiographs are normally taken at times of acute clinical deterioration every 12 to 24 hours, or once NEC 
has been diagnosed, as often as necessary. Two images are usually obtained: a supine image with a vertical beam (patients are flat on their back), and a cross-table lateral image with a horizontal beam, due to concerns of bowel perforations which usually occur within 48 hours of NEC onset (Epelman et al., 2007), (Buonomo, 1999). Although the background of X-ray technology is beyond the scope of this thesis, it is important to note that "excessive number of exposures or very long fluoroscopic times can subject the patient to high exposures [radiation dosages], and the risk versus benefit of the procedure must be considered" (Siedband, 1998). The U.S. Food and Drug Administration (FDA) Center for Device and Radiological Health (CDRH) suggests that X-ray risks with respect to X-ray's ionizing radiation and harm to living tissues are the following (CDHR, 2008), (Center for Devices and Radiological Health (CDRH), 2008):

1. increase in possibility of patient developing cancer later in life

i. risk of cancer increases with larger doses and number of X-ray exams a patient undergoes

ii. risk of cancer is larger for patients undergoing X-ray at a younger age (ex. babies imaged for NEC)

iii. risk of cancer is larger for women than men

2. cataracts and skin burns (at higher levels of radiation exposure)

Therefore, there is a need for a medical imaging modality that would decrease the risks presented by X-ray imaging, but at the same time be as effective, if not better, at detecting NEC in babies. 
Using clinical, laboratory and radiographic features and findings, NEC is further categorized by the 'Bell Staging Criteria' (Bell et al., 1978), (Panigrahi, 2006). Babies suspected of NEC are considered Stage I, definite NEC is considered Stage II and advanced NEC (severe abdominal inflammation or perforated bowels) is Stage III. More information about the Bell Staging Criteria can be found in appendix A, table A-2.

\subsubsection{Management and Prognosis}

NEC is not a simple disease to diagnose. Medical professionals attempt to tackle NEC by trying to diagnose it in its early stage (Stage I), and by taking precautions early on with the population of babies who are at an increased risk of developing it, more specifically premature infants. Constant monitoring of the respiratory function, along with cardiovascular and metabolic function is done for babies suspected of NEC. In addition babies are placed on 'NPO' orders (Latin nil per os, or nothing-by-mouth) (Martin \& Simone, 2006). As McAlmon (2004) suggests, "therapy is based on intensivecare measures and the anticipation of potential problems", and the preferred method of treatment is through medication as opposed to surgical intervention that is required for severe cases. This is because surgical cases and "those with Stage II B and Stage III have a higher incidence of growth delay", as well as being at an increased risk of morbidity and mortality due to infection, respiratory failure, short-bowel syndrome (if parts of intestine were removed due to NEC) among other risks (McAlmon, 2004).

\subsubsection{Populations of interest for NEC}

In order to understand the complex variable relationships in NEC, medicine focuses research interests on two populations: full-term or near-term infants and 
premature ones, more specifically those born before 33 weeks of gestation and those weighing less than $1500 \mathrm{~g}$.

\subsubsection{NEC in full-term or near-term infants}

Unlike the very premature infants, term infants have considerably more mature intestinal and immune systems. Literature review confirms that only a few studies have focused particularly on the larger newborns with NEC. Furthermore, the studies suggest that factors that might place full-term infants at risk of NEC include "exchange transfusion, perinatal asphyxia, maternal preeclampsia, smallness for gestational age, polycythemia, respiratory distress and hypoglycaemia" (Martinez-Tallo, Claure, \& Bancalari, 1997). In a retrospective study of hospital records at the Jackson Memorial Hospital between January 1, 1990 and December 31, 1993 (average delivery of 10,828 deliveries annually), Martinez-Tallo et al. (1997) found that the 'most striking' factors that may set larger infants at risk for NEC are: hypoglycaemia, asphyxia, respiratory problems, CHD (congenital heart disease), exchange transfusion, chorioamnionitis and prolonged rupture of membranes. In addition, the mean age of onset for the group from this study was recorded to be 3.25 days, with gestational age in weeks $38.08+/-2.28$, and birth weight of $2,668+/-718 \mathrm{~g}$.

\subsubsection{NEC in premature infants}

Hallstrom et al. (2003), (2006), also set out to identify laboratory findings predictive of necrotizing enterocolitis. While conducting an epidemiological study on a population of 117,892 newborns from the Tampere University Hospital (Finland), they found that $18.3 \%$ of the babies diagnosed with NEC were at less than 33 weeks of 
gestation. Statistical analysis performed by the group further showed the day of onset of NEC symptoms to be 4 , with gestational age in weeks $26+/-2$, and birth weight $952 \mathrm{~g}+/-$ 322.4g. From their study, the group found that metabolic acidosis, platelet count, blood glucose, leukocyte levels and $\mathrm{pH}$ were useful indicators of NEC in preterm infants (Hallstrom, Koivisto, Janas, \& Tammela, 2006). In addition, according to Stall, as reported by (Di Lorenzo, 2000), case fatality rates for infants weighing less than $1500 \mathrm{~g}$ range from 10-44\%; and those infants weighing more than $2500 \mathrm{~g}$ range from $0-20 \%$ ". The mortality rate increases to $35-50 \%$ for infants weighing less than $1000 \mathrm{~g}$, and these NEC babies having serious long-term complications ranging from poor growth to poorer neuro-developmental outcomes (Luig \& Lui, 2005b).

\subsection{Problem Statement}

In response to the variation of pathological signs and symptoms in NEC, there is a growing need to develop a risk assessment model or a scoring system tool that will allow doctors to detect NEC much earlier, and do so for different populations of interest. Combining the hidden and valuable relationships in the medical data that are not necessarily transparent to medical professionals, with the computational power of the ANN RFW data mining tool, the goal is to assist medical professionals in outcome prediction of NEC in the neonatal intensive care units (NICU). In addition, the predictive capabilities of the ANN RFW will be compared to another classifier - the Support Vector Machine (SVM). 


\subsection{Thesis Objectives}

This thesis has 3 objectives:

1) Analyze the performance of MIRG's ANN RFW in prediction of NEC, by examining the relationships between variables in day 1 data and developing a day 1 model for NEC for the following populations:

a. Total Canadian Neonatal Network database

b. 2 risk group subsets of NEC

- Babies weighing $<1500 \mathrm{~g}$ (VLBW)

- Babies with gestational age $<33$ weeks

2) Analyze the performance of SVM in prediction of NEC, by examining the relationships between variables in day 1 data and developing a day 1 model for NEC for the following populations:

c. Total Canadian Neonatal Network database

d. 2 risk group subsets of NEC

- Babies weighing $<1500 \mathrm{~g}$ (VLBW)

- Babies with gestational age $<33$ weeks

3) Compare the predictive power of MIRG's ANN RFW tool to that of SVM for the outcome of NEC. 


\subsection{Thesis Outline}

The scope of this research is covered in 5 chapters.

Chapter 1: Introduction covers significance and research motivation. In order to establish the problem statement and thesis objectives, a medical impact section is introduced to present the seriousness of the medical complication (NEC) of central interest to this research.

Chapter 2: Literature Review covers the concept of data mining in medicine, and looks at the current neonatal scoring models that are used in outcome prediction in medicine. The outcome prediction models cover basic modeling attempts of NEC as well. As part of Clinical Decision Support Tools, ANNs and SVMs are examined next. Lastly, the Canadian Neonatal Network (CNN) database used in this research is introduced along with a brief analysis of issues with respect to database preparation.

Chapter 3: Methodology covers steps taken in preparing the CNN database for modeling NEC. Dataset creation is covered for both the ANN RFW and SVM tool and is done for the 3 populations of interest: total database, VLBW and gestation age less than 33 weeks. The approach used in reaching the minimum data set is established for both tools used. 
Chapter 4: Results and Discussion present the results obtained for all of the NEC models examined in this research. This is done for the ANN RFW tool as well as the SVM tool. Model comparison is also done between the tools with respect to their performance and minimum data sets.

Chapter 5 Conclusion summarizes the work and findings of this thesis and provides a summary of contributions to knowledge and future research. Future research is specifically aimed at NEC. 


\section{LITERATURE REVIEW}

Vast amounts of data are collected daily. The overwhelming task is in maximizing the use of the available information and deriving meaning from it - knowledge discovery $(\mathrm{KD})$. The concept of knowledge discovery and data mining (DM) is used hand-in-hand. Data mining is a rapidly growing discipline used in several fields of study: banking and finance, telecommunications, engineering, medicine, pharmacology and sciences (Klosgen \& Zytkow, 2002). It is seen as "the process of discovering meaningful new correlations, patterns and trends by sifting through large amounts of data stored in repositories, using pattern recognition technologies as well as statistical and mathematical techniques" (Larose, 2005). Hand et al. (2001) also define data mining as "the analysis of (often large) observational data sets to find unsuspected relationships and to summarize the data in novel ways that are both understandable and useful to the data owner". The process of data mining and knowledge discovery is often referred to as

DMKD. Review of literature and relevant concepts focuses on data mining in medicine with regards to the neonatal severity of illness scoring systems and the use of Clinical Decision Support Systems (CDSSs). CDSSs of interest include the Artificial Neural Networks (ANNs) and Support Vector Machines as part of the data mining cycle. Data preparation for the data mining process is also discussed.

\subsection{Data mining in medicine}

With respect to the field of medicine, data mining shows remarkable promise. Health care facilities collect patient information on a daily basis, especially for critical patients such as those in the intensive care units. Data collected includes demographic 
information, numerical measurements which include lab results and vital signs, as well as recorded signals such as ECG (electrocardiogram) and radiological images. This information is useful for several reasons (Cios \& Moore, 2002), (Perreault \& Metzger, 1999) :

1. It forms the basis of a patient's medical record history and legal record.

2. It supports communication between health care providers.

3. It identifies deviations from expected trends which is helpful in early diagnosis, as well as patient's prognosis.

4. It supports medical research: based on already existing cases, future cases exhibiting similar trends can be diagnosed.

In response to the complexity of data mining and the lack of a knowledge discovery procedure, in 1996 analysts representing DaimlerChrysler, SPSS and NCR developed a 6-stage standard DMKD process model that is industry-neutral, tool-neutral and application-neutral (Larose, 2005). Using the CRISP-DM model as foundation, Cios and Moore (2002) developed a supplementary data mining model applicable specifically to the field of medicine (figure 2.1).

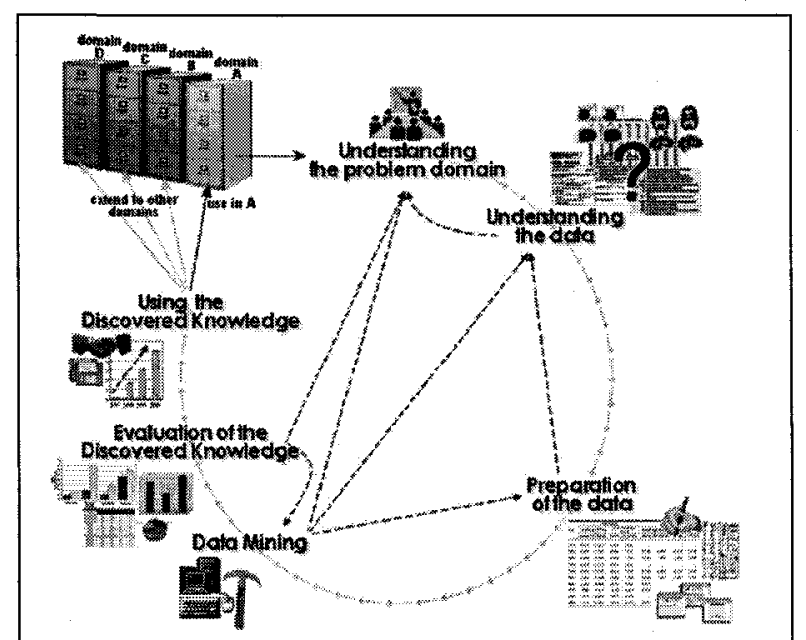

Figure 2.1: The six-step DMKD process model (Cios \& Moore, 2002) 
The life cycle consists of 6 phases, which are adaptive and dependent on each other. 'Adaptivity' in this process means that the next phase in the sequence can, and does, depend on the outcome(s) associated with a preceding phase (Larose, 2005). The outer circle of the six-step model indicates that the solution to a problem is of iterative nature, as research problems often raise further questions of interest (Larose, 2005). Additional feedback loops to account for changes and decisions made in a particular step and their impact on later steps of the DMKD process (Cios \& Moore, 2002).

When developing a DMKD model, it is essential that in the early stages of research emphasis be on understanding the problem domain and data steps of the cycle. This is followed by the labour intensive data preparation and data mining stages. To ensure that results obtained in the modeling stage are valid for the domain of interest, evaluation of the discovered knowledge evaluation is done next. The evaluation stage of the six-step DMKD would have to undergo a clinical study, similar to the one performed by Townsend (2007), where she conducted a survey and performed an analysis on physicians' ability to accurately identify high, medium and low risk patients with respect to different medical outcomes. Once the results are validated by medical professionals through rigorous medical trials, deployment of the model would be considered (Dorling et al., 2005), (Garg et al., 2005). Cios and Moore have successfully applied their DMKD process model to several medical domains, in particular the automation of the cardiac SPECT (Single-Photon Emission Computer Tomography) diagnosis (Sacha, Cios, \& Goodenday, 2000), (Kurgan, Cios, Tadeusiewicz R., Ogiela, \& Goodenday, 2001). 


\subsection{Neonatal (Severity of Illness) Scoring Systems}

In an effort to asses the illness severity of newborns, neonatal scoring systems are used extensively in neonatal intensive care units. Also known as neonatal illness/disease scoring systems, scoring systems are epidemiologic tools that attempt to standardize outcome comparison between heterogeneous groups of patients across different hospital environments (Dorling et al., 2005), (Dorling \& Field, 2008). Relying on the physiological, demographic, and clinical data collected on newborns, the scoring systems are largely used for mortality and morbidity risk assessments, in addition to other group and individual predictions, as summarized in table 2.1.

Table 2.1: Research uses of predictive scores in neonatology (Dorling et al., 2005)

\section{Group predictions}

1. Comparing study groups for similarity of risk

2. Auditing the severity of illness in different units

3. Comparing performance of different units

4. Determining trends in results over time

5. Reviewing if infants are treated appropriately for risk (e.g. number of septic screens or ventilation days)

6. Comparing rates of complications; are some preventable?

\section{Individual predictions}

1. Giving prognostic information

2. Stratifying infants in trials (to ensure similarity of risk)

3. Determining individual treatment

Of the several systems that are in use today, the most popular ones are the Apgar score, CRIB, NTISS, SNAP (SNAP-II, SNAPPE-II). Dorling et al. (2005) published a review of these systems in which the authors note that: "A balance needs to be drawn between a complex score including many variables, and therefore difficult to complete, and a simpler model that may be easier to use but not as accurate". Although speaking with respect to morbidity, the authors point out that no score can fully quantify the complex factors that make up an infant's outcome (Dorling et al., 2005). 


\subsubsection{The Apgar Score}

Developed by Dr. Virginia Apgar in 1953, the Apgar Score is a method of evaluating a newborn's condition at birth (Apgar, 1953). It is based on five characteristics: skin colour, heart rate, reflex irritability, muscle tone and breathing. Physicians have adopted the acronym of 'APGAR': Appearance, Pulse, Grimace, Activity, and Respiration respectively. Scored between 0 (min) and $10(\max )$ and assessed at 1min and 5mins, Apgar score of less than 7 is often associated with a higher risk of mortality (Richardson, Corcoran, Escobar, \& Lee, 2001). Out of the four scoring systems discussed, Apgar is the most subjective one, as it relies more on the opinions and assessment of the medical professional rather than being based on the physiological data. Appendix B shows how the Apgar score is calculated.

\subsubsection{CRIB}

The Clinical Risk Index for Babies, CRIB, is a mortality scoring system for infants born at less than 32 weeks of gestation or weighing less than or equal to $1500 \mathrm{~g}$. It is a logistic regression model where parameters are weighted according to their statistical relation to death (The International Neonatal Network, 1993). The development of the CRIB score was performed on data from four UK teaching hospital neonatal units between July $1^{\text {st }}, 1988$ and June $30^{\text {th }}, 1990$, on a total of 1548 infants. The score is based on data collected in the first 12 hours of admission for the following six variables: birth weight, gestational age, maximum and minimum fraction of inspired oxygen, maximum base excess and presence of congenital malformations (appendix B). The major advantage of CRIB is the ease of data collection necessary to calculate the score, which 
approximately takes 5 minutes; in addition, since the CRIB score is determined in the first 12 hours of life, it is less susceptible to treatment effects compared to other models (Dorling et al., 2005).

Since its original development, CRIB has undergone modifications: CRIB II (Parry et al., 2003). The new model uses a grid system (appendix B) predicting mortality by gestational age along with birth weight, in addition to admission temperature and base excess. The intent is to improve predictions for very premature babies and to exclude variables that are influenced by the primary care provider (Dorling et al., 2005).

\subsubsection{NTISS}

The Neonatal Therapeutic Intervention Scoring System, NTISS, was created by modifying the existing adult-version TISS (Cullen, Civetta, Briggs B.A, \& Ferrara, 1974), and making it suitable for neonatal intensive care (Cullen et al., 1974), (Gray, Richardson, McCormick, Workman-Daniels, \& Goldmann, 1992). There are 62 NTISS variables, for which the scores are assigned from 1 to 4 with respect to various intensive care therapies. Gray et al. (1992) found that for the 1643 newborns in their study, between November $1^{\text {st }}, 1989$ to September $30^{\text {th }}, 1990$, independent of birth weight, NTISS was a valid measure of neonatal illness severity and resource utilization (Gray et al., 1992). Appendix B shows how the NTISS score is calculated. Unlike other scoring systems, NTISS is based on the treatments received by an infant rather than the measure of physiological factors (Dorling et al., 2005). 


\subsubsection{SNAP}

Unlike the CRIB scoring system, the Score for Neonatal Acute Physiology (SNAP) can be used on all admissions regardless of gestation age or birth weight. SNAP, developed by Richardson et. al (1993), uses 26 variables as outlined in appendix A, for which data is collected in the first 24 hours. Ideally, SNAP's intent is to "score the worst physiologic derangements in each organ system in the first 24 hours" (Richardson et al., 1993). However, the drawbacks of the initial SNAP scoring system is the large number of variables (26) that it needs for calculations, which increases its complexity. Also, the variables are assigned a weight of $0,1,3$ or 5 , as predetermined by expert opinion (Dorling et al., 2005). An additional drawback noted by Dorling et. al (2005) suggests that because the original SNAP model was derived from a population with a very low number of VLBW babies, it has a reduced sensitivity to differences between the most premature infants. Richardson et. al also confirmed this drawback (1993).

In order to compensate for its drawbacks, Richardson et. al. added three more variables to the SNAP model (Perinatal Extension variables): birth weight, small for gestational age (SGA) and 5min Apgar score. Using logistic regression the authors reduced the original SNAP variable set to include only 6 variables, thus developing SNAP-II and SNAPPE-II models as shown in appendix B (Richardson et al., 2001).

\subsubsection{NEC Scoring Systems}

Although attempts have been made to use scoring systems designed strictly for mortality for predictions of adverse medical outcomes such as chronic lung disease, research suggests that these models perform less well (Dorling et al., 2005). Ideally it 
would be preferred if the same models that predict mortality could be used for other medical events; however, medical events are not governed by the same factors. This is not different for NEC. Two NEC risk assessment / scoring systems were found: NECMortality Scoring System and Mathematical modeling in NEC.

\subsubsection{Presurgical NEC-mortality scoring system}

In response to the need of improving NEC outcome, Kessler et. al. (2006) set out to asses the relationship between early-laboratory parameters, disease severity, type of management (surgical or conservative) and outcome in necrotizing enterocolitis. The study includes neonate data collected between 1980 and 2002 at the University Children's Hospital in Mainz, Germany. The parameters recorded were: birth weight (BW), gestational age (GA), sex, type of treatment, severity of NEC (Bell staging criteria), pre-intervention blood parameters (blood cell counts, haemoglobin concentration, lactate, C-reactive protein) and survival (Kessler et al., 2006). Statistical analysis between the NEC and normal groups was done using unpaired t-test, where $\mathrm{p}<0.05$ was considered statistically significant. Table 2.2 summarizes statistical information for the 128 NEC babies eligible for the study.

Table 2.2: Statistical summary of Kessler et. al (2006) study

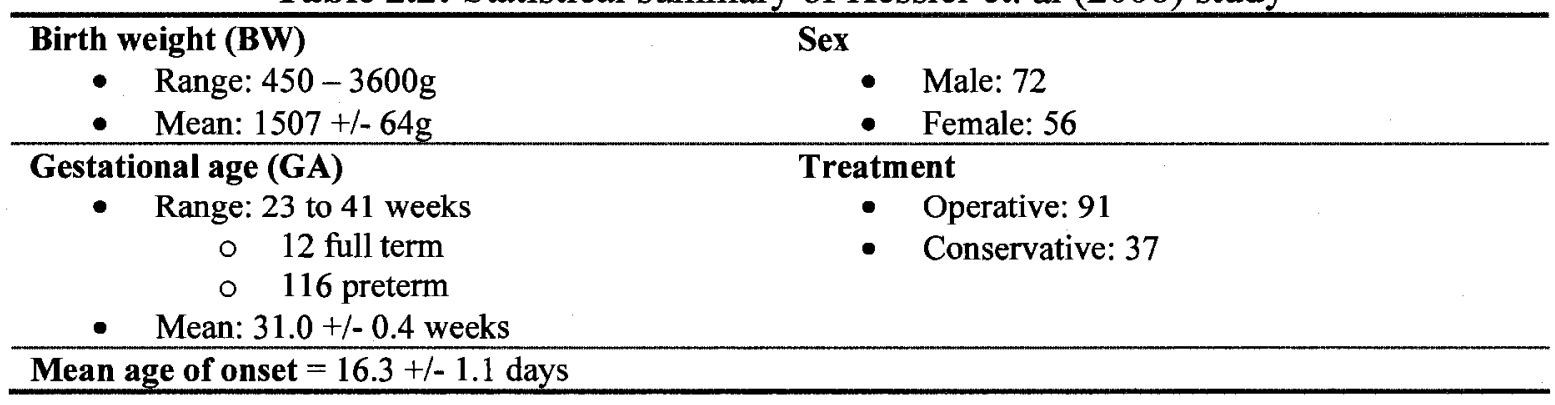


In addition to comparisons between surgically treated and medically treated (conservative) infants, a comparison is done between NEC survivors and non-survivors. The findings are summarized in tables 2.3 and 2.4 respectively.

Table 2.3: Laboratory values, survival and NEC-score in operated and conservatively treated infants (Kessler et al., 2006)

\begin{tabular}{|c|c|c|c|c|c|}
\hline \multirow[b]{2}{*}{ Parameter } & \multicolumn{2}{|c|}{ Operated } & \multicolumn{2}{|c|}{ Conservative } & \multirow[b]{2}{*}{ P-value } \\
\hline & $N^{a}$ & Value & $N^{a}$ & Value & \\
\hline Survival (\%) & 78 & 82.8 & $>0.05$ & - & - \\
\hline Leukocyte count $\left(\times 10^{9} / \mathrm{l}\right)$ & 86 & $10.6+/-1.0$ & 34 & $14.9+/-1.0$ & $>0.05$ \\
\hline Hemoglobin $(\mathrm{g} / \mathrm{l})$ & 64 & $13.6+/-0.3$ & 21 & $12.8+/-0.4$ & $>0.05$ \\
\hline Platelet count $\left(\times 10^{9} / \mathrm{l}\right)$ & 86 & $213+/-14$ & 34 & $221+/-27$ & $>0.05$ \\
\hline $\mathrm{CRP}(\mathrm{mg} / \mathrm{l})$ & 85 & $46.8+/-4.4$ & 33 & $43.6+/-8.1$ & $>0.05$ \\
\hline Lactate (mg/dl) & 76 & $3.6+/-0.6$ & 27 & $3.0+/-0.8$ & $>0.05$ \\
\hline${ }^{* *} N E C$-score (Table 2.5) & 66 & $4.1+/-0.3$ & 31 & $4.1+/-0.2$ & $>0.05$ \\
\hline
\end{tabular}

From table 2.3 , it can be seen that the percentage of survivals did not differ between operated and conservatively treated babies. Of the parameters of interest, only the leukocyte counts differ significantly between operated and conservatively treated babies (Kessler et al., 2006). In conclusion, the model is not useful for prediction of babies that need operative versus those that need surgical treatment.

Table 2.4: Laboratory value, BW, GA and NEC-score in survivors and nonsurvivors (Kessler et al., 2006)

\begin{tabular}{|c|c|c|c|c|c|}
\hline \multirow[b]{2}{*}{ Parameter } & \multicolumn{2}{|c|}{ Survivors } & \multicolumn{2}{|c|}{ Non-survivors } & \multirow[b]{2}{*}{ P-value } \\
\hline & $N^{a}$ & Value & $N^{a}$ & Value & \\
\hline $\mathrm{BW}(\mathrm{g})$ & 103 & $1568+/-71$ & 25 & $1199+/-125$ & $<0.01$ \\
\hline GA (weeks) & 103 & $31+/-0.4$ & 25 & $28.7+/-0.8$ & $<0.0001$ \\
\hline Leukocyte count $\left(\mathrm{x} 10^{9} / 1\right)$ & 96 & $11.2+/-0.9$ & 25 & $13.0+/-1.6$ & $>0.05$ \\
\hline Hemoglobin $(\mathrm{g} / \mathrm{l})$ & 65 & $13.4+/-0.3$ & 20 & $13.3+/-0.5$ & $>0.05$ \\
\hline Platelet count $\left(\times 10^{9} / 1\right)$ & 96 & $220+/-14$ & 25 & $187+/-27$ & $>0.05$ \\
\hline $\mathrm{CRP}(\mathrm{mg} / \mathrm{l})$ & 87 & $47+/-3.7$ & 24 & $41+/-3.7$ & $>0.05$ \\
\hline Lactate $(\mathrm{mg} / \mathrm{dl})$ & 81 & $2.6+/-0.25$ & 23 & $7.0+/-1.8$ & $<0.05$ \\
\hline **NEC-score (Table 2.5) & 79 & $3.7+/-0.18$ & 18 & $5.7+/-0.32$ & $<0.001$ \\
\hline
\end{tabular}

${ }^{\mathrm{a}} \mathrm{N}=$ number of patients for whom results are available, $\mathrm{CPR}=\mathrm{C}$-reactive protein

**NEC-score (Table 2.5) - score calculated subsequent development of the scoring system 
Results in table 2.4 indicate that BW, GA, and lactate levels are all significantly lower in infants who died than those who survived. Adding the value of Bell Staging Criteria to their results, and assigning scores based on expert medical opinion, table 2.5 was developed. Points are scored based on gestational age, Bell Stage, platelet count and lactate levels. Healthy babies would ideally score 0 points, whereas the extremely sick ones can score up to 8 points. Applying the NEC-score to the 128 NEC neonates that were a part of the study, the authors found that the score of 4.5 out of 8 was the 'optimal cut-off' with respect to NEC-mortality, i.e. babies scoring more than 4.5 were more likely to die from NEC. The sensitivity and specificity for predicting survival using the NECscore was 0.71 and 0.72 , respectively (Kessler et al., 2006). The conclusion: "The predictive potential for survival was superior using the score than using single parameters" (Kessler et al., 2006).

Table 2.5: NEC-score (Kessler et al., 2006)

\begin{tabular}{lccc}
\hline & \multicolumn{3}{c}{ Score } \\
\cline { 2 - 4 } Parameter & $\boldsymbol{0}$ & $\boldsymbol{1}$ & $\mathbf{2}$ \\
\hline GA (weeks) & $>32$ & $30-32$ & $<30$ \\
Bell stage & I & II & III \\
Platelets $(\mathrm{g} / \mathrm{l})$ & $>200$ & $150-200$ & $<150$ \\
Lactate $(\mathrm{mg} / \mathrm{d})$ & $<2.5$ & $2.5-3.3$ & $>3.3$ \\
\hline
\end{tabular}

However, several issues with this scoring system need to be noted:

1. Data set is relatively small, 128 babies, and some patients have missing data for variables that were analyzed. The authors chose not to impute or remove cases with missing variables.

2. There is a significant number of premature babies (116) with NEC compared to infants considered to be born at term (12). 
3. This is a single hospital neonatal cohort, with a span of 20 years, and might now be representative of the population across the entire country.

4. This scoring system has not been properly validated.

\subsubsection{Mathematical model of inflammation in NEC}

Upperman et. al (2007) attempted to develop a "calibrated mathematical model of inflammation and its pathologic consequences in NEC" to predict physiologic and biologic response in infants suffering from it. The researchers focused on developing a model using various biomarkers such as proinflammatory cytokines and products of vasoactive substances present in serum and intestine of infants with NEC. This model is based on system biology, which integrates experimental and computational tools to characterize complex biological phenomena (Upperman et al., 2007). Research focused on contributions of cellular and molecular mechanics to the pathogenesis of NEC. The mathematical model developed by Upperman et. al. incorporates two system compartments: tissue and blood. These are further broken down into smaller pieces to allow for mathematical analysis. For example (equation 2.1), one of the sub-systems developed models the interaction of the active neutrophils in the tissue and blood level of inflammatory cytokines (proteins).

$$
\begin{gathered}
n_{a}^{\prime}=f\left(c_{\mathrm{b}}\right)-v \cdot n_{\mathrm{a}} \\
c_{b}^{\prime}=\gamma \cdot c_{\mathrm{b}}+\frac{\mathrm{d}}{\mathrm{d}+\eta}\left(r \cdot n_{a}-\eta \cdot c_{\mathrm{b}}\right)
\end{gathered}
$$

where,

$n_{a}=$ active neutrophils in the tissue

$c_{b}=$ blood level of inflammatory cytokines

$f\left(c_{b}\right)=$ sigmoidal function representing combined production of activated neutrophils by inflammatory cytokines \& transport of activated neutrophils to the tissue compartment 
$v, \gamma, \eta=$ parameters of degradation rates of neutrophils, blood cytokines and tissue cytokines respectively

$r=$ rate of production of cytokine by neutrophils in the tissue

$d=$ diffusivity of cytokines from the blood to the tissue

The complexity of this model is beyond the scope of this research. Furthermore, this has yet to be validated in a clinical setting.

\subsection{Clinical Decision Support Systems}

While data mining centres around problem solving by analyzing existing data, decision support centres around development of systems aimed at assisting decision makers in problem solving and decision making (Mladenic, Lavrac, Bohanec, \& Moyle, 2003). Clinical Decision Support Systems (CDSSs) in particular use data mining techniques to assist physicians and other health care professionals with decision making tasks. Although the debate over the medical usefulness of CDSS (Berner, 1998)s with respect to practitioner performance and patient outcomes is on-going, existing trials of CDSSs include: systems for diagnosis, systems for prevention, systems for disease management, systems for drug dosing and drug prescribing (Garg et al., 2005), (Berner, 1998). To properly assess the value of a system, model evaluation techniques need to be established first. Systems that 'learn' by example and over time, i.e. non-knowledge based system such as neural networks and support vector machines, are then discussed.

\subsubsection{Model Evaluation Techniques}

Frequently used performance measures of data mining techniques include the constant predictor, sensitivity, specificity, classification rate (CR), receiver operating characteristic curve (ROC), and the stopping criterion. 


\section{Confusion Matrix}

A confusion or contingency $n \times n$ square matrix summarizes the dispositions of true and false positive and negative instances, and is used to evaluate the performance of a classification system (Fawcett, 2006). Table 2.6 is 2-by-2 square matrix, used for a 2class classification system, such as the one used in this research. In medicine, a less frequent outcome is associated with the positive classification $(+1)$. The 'normal' outcome is associated with the negative classification $(-1)$.

Table 2.6: $2 \times 2$ confusion matrix for a 2-class classification system

\section{Actual Class}

\begin{tabular}{clc|c}
\cline { 2 - 3 } & & Positive (P) & Negative (N) \\
\hline \multirow{2}{*}{$\begin{array}{c}\text { Predicted } \\
\text { Class }\end{array}$} & Positive & True Positive (TP) & False Negative (TN) \\
\cline { 2 - 4 } & Negative & False Positive (FP) & True Negative (TN) \\
\hline
\end{tabular}

\section{Constant Predictor and Priori Knowledge}

In order to ensure consistency with respect to comparisons between various data mining methods, a priori knowledge about the data distribution needs to be established first. A priori knowledge refers to the actual number of positive versus negative cases represented in the population of interest.

$$
\begin{aligned}
& \text { Positive priori outcome }=\frac{T P+F N}{(T P+T N+F P+F N)} \\
& \text { Negative priori outcome }=\frac{\mathrm{TN}+\mathrm{FP}}{(\mathrm{TP}+\mathrm{TN}+\mathrm{FP}+\mathrm{FN})}
\end{aligned}
$$

Furthermore, the Constant Predictor $(C P)$ is established by using a prior knowledge. CP is set as the negative priori outcome i.e. the value which is associated with the 'normals' 
in a population of interest. For example, if $95 \%$ of the population is considered to be healthy, while $5 \%$ is sick, the CP would be $95 \%$.

\section{Sensitivity and Specificity Analysis}

Using the data from the confusion matrix, sensitivity, specificity and classification rate are also calculated. Sensitivity is defined as the true positive rate, and is also known as a hit rate or a recall.

$$
\text { Sensitivity }=\frac{T P}{(T P+F N)}
$$

Specificity, on the other hand, is the measure of the true negative rate.

$$
\begin{gathered}
\text { Specificity }=\frac{T N}{(T N+F P)} \\
O R \\
\text { Specificity }=1-\text { false positive rate }=1-\frac{F P}{(F P+T N)}
\end{gathered}
$$

\section{Classification Rate}

The classification rate, also known as accuracy, is the overall success rate with respect to the number of correct classifications, both positive and negative, over the entire population (Witten \& Frank, 2005). For imbalanced medical data, where negative a priori is much higher than positive a priori, $\mathrm{CR}$ measure is not a good indicator of performance (Maimon \& Rokach, 2005). MIRG has found that CR is an accurate 
measure of performance only in cases where the desired outcome is above $15 \%$ (Frize et al., 1998).

$$
\text { Classification rate }=\frac{T P+T N}{T P+T N+F P+F N}
$$

\section{Receiver Operating Characteristic (ROC) Curve}

According to Fawcett (2006) "A receiver operating characteristic (ROC) graph is a technique for visualizing, organizing and selecting classifiers based on their performance". In addition, ROC curves are popular in medical decision making where they are used in assessment of risk scores (Dorling \& Field, 2008). An ROC curve is generated by plotting the true positive rate, i.e. the sensitivity, versus the false positive rate, or 1 - specificity. It is the area under the curve (AUC) that is of most interest in analysis. Figure 2.2 is an example of an ROC graph showing five (A, B, C, D, E) discrete classifier points, with corresponding (false positive rate, true positive rate) coordinate pairs.

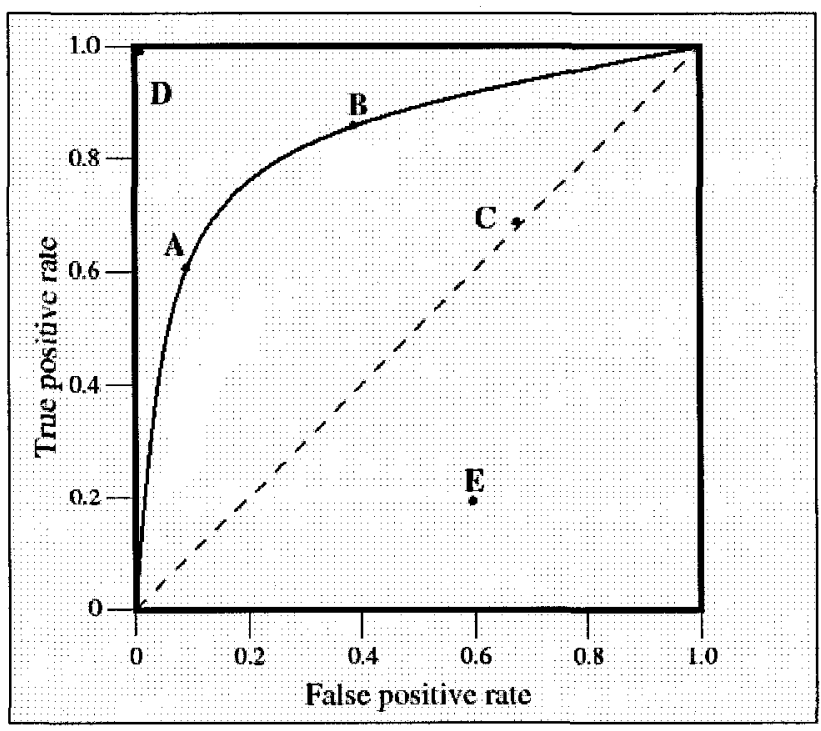

Figure 2.2: An ROC graph (Fawcett, 2006) 
With respect to (TP rate, FP rate) coordinate pairs, the general assumption is that one point is better than the other if it is located in the 'northwest' corner of the graph, maximizing the TP rate and minimizing the FP rate (Fawcett, 2006). The ideal point on the ROC curve is represented by point $\mathrm{D}(0,1)$, where all positive samples are identified correctly and no negative examples are misclassified. As such, point D is considered a 'perfect' classifier. 'Conservative' classifiers, those in the proximity of the bottom lefthand side, have lower FP errors but often have low TP rates as well. On the diagonally opposite end, classifiers within proximity of $(1,1)$ coordinate pair are considered more 'liberal' as they classify nearly all positives correctly, but also have a high rate of false positives. Point A compared to point B is more conservative; however, point A might be a more appropriate classifier as it has a lower rate of false positives. This depends on the application and on the balance of training samples present in each class (Maimon \& Rokach, 2005). Any classifier located on the line $y=x$, ex. point $C$, represents a 'random guess' classifier and is considered useless. A classifier worse than a random guess is the one identified by point $\mathrm{E}$, and in general any classifier found below the $\mathrm{y}=\mathrm{x}$ line. With respect to the value of the ROC curve in medicine, medical literature reports that any scoring systems with a value for the area under the ROC curve yielding $80 \%$ and greater, may be useful in clinical practice (Dorling et al., 2005), (Dorling \& Field, 2008).

\section{Stopping Criteria}

In order to ensure that overtraining and overfitting (memorizing) of data is not taking place, stopping criteria is used to identify the optimal performance of data mining algorithms (Ennett, 2003). Classification rate and the minimum square error are often 
used as a stopping criteria; however, due to the sensitive nature of medical problems, where it is of higher priority to correctly identify the outcomes of rare occurrence, the preferred stopping criteria was found to be the logarithmic-sensitivity index (logsens) (Scales, 2001; Ennett, 2003). Unlike the classification rate and minimum square error, the logsens index strives to find an optimal balance between the sensitivity and the specificity classifications, while slightly favouring the higher sensitivity at the expense of specificity (Scales, 2001). By varying the value of $n$, the weight in favour of sensitivity is monitored. This is currently used by MIRG for the ANN RFW tool.

$$
\text { logsens }=- \text { sensitivity }{ }^{\mathrm{n}} \log _{10}(1-\text { sensitivity } * \text { specificity })
$$

\subsubsection{Artificial Neural Networks}

Mimicking the function of the biological neural networks, because of their nonknowledge-based learning abilities artificial neural networks (ANNs) are gaining attention as classification algorithms (Berner, 1998). Viewed as an adaptive machine, Haykin (1999) defines the neural network as:

"A neural network is a massively parallel distributed processor made up of simple processing units, which has a natural propensity for storing experiential knowledge ad making it available for user. It resembles the brain in two respects:

1. Knowledge is acquired by the network from its environment through a learning process.

2. Interneuron connections strengths, known as synaptic weights, are used to store the acquired knowledge. "

The smallest information processing unit of the neural network is the neuron, as seen in figure 2.3. The three basic elements of the neuron model are (Haykin, 1999):

1. The 'synapses', characterized as the weights $\left(w_{\text {in }}\right)$ which lie in the range that includes both positive and negative numbers. 
2. The 'adder', which sums the input signals $\left(x_{l} \quad \ldots x_{n}\right)$ as they are weighed by their respective weights $\left(w_{i I} \quad \ldots w_{i n}\right)$, in addition to the externally applied bias, which increases/decreases the input to the activation function.

3. The 'activation function' or the transfer function, which limits the amplitude of the output of the neuron.

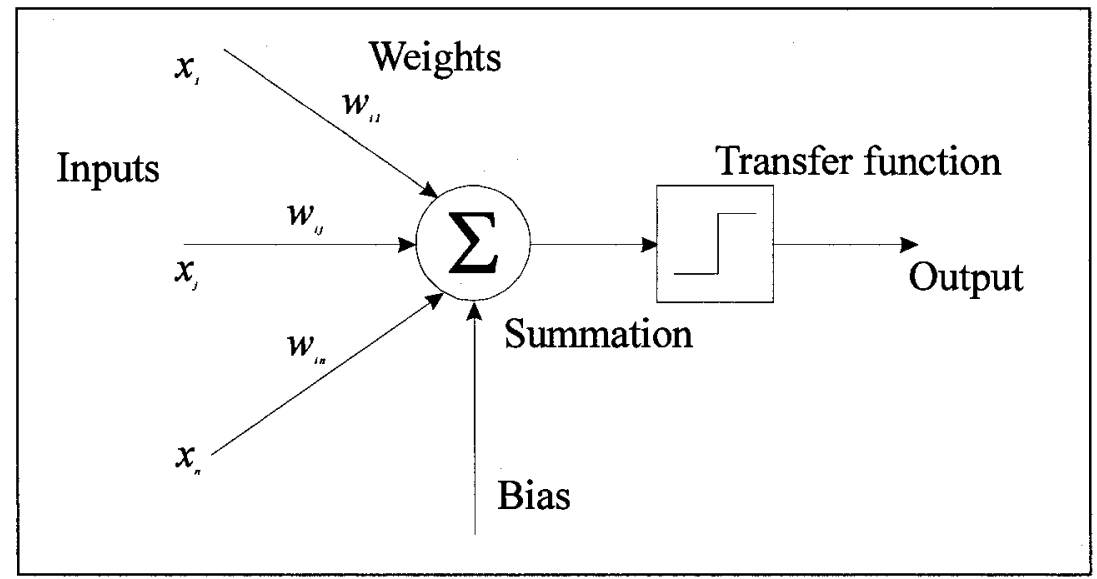

Figure 2.3: Information processing unit - the neuron (Pan, 2002)

The output of the neuron $i$, based on figure 2.3 , is given as:

$$
\text { Output }=\text { TransferFunction }\left[\left(\sum_{j=1}^{\mathrm{n}} \mathrm{w}_{\mathrm{i}} \mathrm{X}_{\mathrm{j}}\right)+\text { bias }\right]
$$

Multiple neurons are further grouped together to form networks which can be made up of different structures. Haykin (1999) identifies three fundamentally different classes of neural network architectures (structures): single-layer feedforward, multi-layer feedforward and recurrent networks. Further classification can be done based on the type of learning algorithm used to train the network (supervised, unsupervised, recurrent), as well as the application, connection type and computational modeling (Haykin, 1999).

Of interest to this research are the single and multi-layer neural networks (classifier networks), as seen in figure 2.4. 


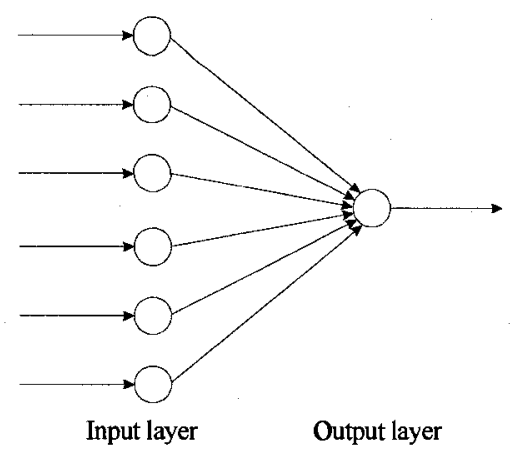

(a)

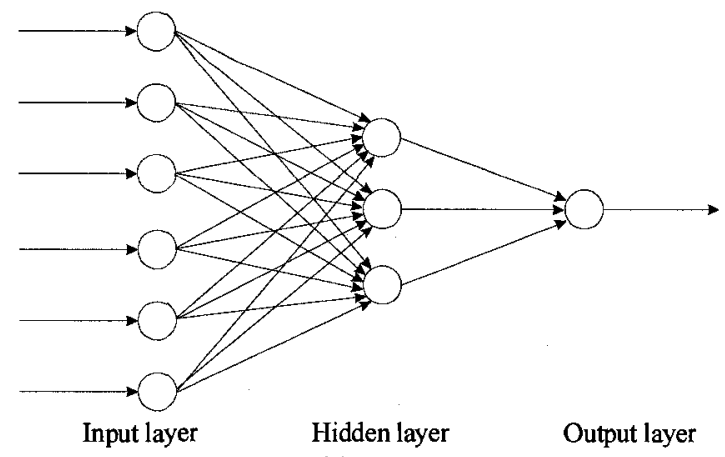

(b)

Figure 2.4: (a) 2-layer network; (b) 3-layer network

The networks can also be fully connected, figure $2.4(\mathrm{~b})$, where each node in the layer is connected to a node in the subsequent layer; or, connections can be partial so that not every input connects to every subsequent node. The type of connections shown in figure 2.4 make up a feedforward neural network, where information flow is in only one direction: from the input nodes, through the hidden layer (if it exists), ending at the output layer. In general, the computing power of the ANNs is achieved through the parallel distributed structure, in addition to the network's ability to learn and generalize outputs for inputs not encountered during the training (Haykin, 1999), (Hertz, Krogh, \& Palmer, 1991). The input-output mapping is attained with a learning paradigm known as supervised learning, where each sample from a training set and its input signals map to a desired response (outcome). This also makes the network adaptable, as retraining is necessary to account for changes in the operating environment (Haykin, 1999). By modifying the weights during training, the differences between the expected response and the actual response are minimized. ANNs using the backpropagation learning algorithm attempt to minimize the error (mean squared error), by updating the connection weights and biases after the error is propagated backwards through the network (Haykin, 1999). 
In addition to the 'classifier' multi-layer neural network used in this thesis, computational models include association, optimization and self-organization networks (Haykin, 1999). An example of an associative network is the Hopfield model, which is a symmetric recurrent architecture ANN, i.e. the data flow is bidirectional. The units of this network take on binary thresholds values for their states, as determined by whether or not the unit input exceeds a certain threshold (Hertz et al., 1991). The Boltzmann Machine is an example of an optimization network, which makes use of stochastic transfer functions and introduces 'random' variations into the network (Haykin, 1999). Self-organizing networks such as the Kohonen network use a form of unsupervised learning (network provided with inputs, but not outputs). The network 'decides' what features are used to group input data (Haykin, 1999).

\subsubsection{Transfer functions}

There are various types of activation functions used for ANN training: threshold function (step function), piecewise-linear function, and sigmoid function. The type of activation function used determines if the network model is linear or non-linear, regardless of the number of hidden layers. For example, multi-layer networks with fixed weights and a linear activation function are equivalent to a 2-layer network (Russell, 1993), (Haykin, 1999). "A linear system with any number $n$ of layers is equivalent to a single-layer linear system whose weight matrix is the product of the $n$ intermediate weight matrices" (Russell, 1993). If a simple linear model is desired, the activation function used has to be linear. However, non-linear mapping functions are used more often for multi-layer networks as they provide more computational capability (Haykin, 1999), (Rybchynski, 2005). MIRG uses the non-linear hyperbolic transfer function for its 
multi-layer ANN tool, equation 2.8 and figure 2.5, because of the sharper transition between output values which permits faster learning (Buskard, 1994), (Ennett, 2003).

$$
f(x)=\frac{e^{x}-e^{-x}}{e^{x}+e^{-x}}=\frac{e^{2 x}-1}{e^{2 x}+1}
$$

The function ranges $(-1,1)$, and offers a sharp sigmoid-like transition thus minimizing output values near and at zero (Fausett, 1994). As such, the output of the neural network is either on the -1 side of the 1 side of the threshold value (zero).

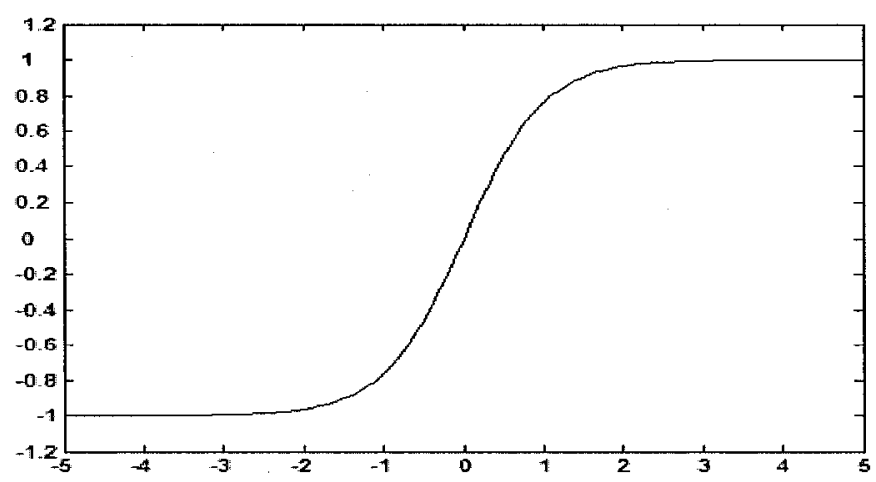

Figure 2.5: Hyperbolic transfer function

\subsubsection{ANN RFW tool \& driving parameters}

ANN Research Framework Tool (RFW), developed by MIRG, uses nine driving parameters for the feedforward backpropagation algorithm used for training of the network. These parameters control the rate at which the weights and the biases of the network are updated, while attempting to minimize the error between the actual and the desired outputs. MIRG researcher, Eliane Charette worked on automation of the driving parameters for the ANN RFW tool in order to improve the performance with respect to speed and calculation of optimal parameters (Frize, Ennett, \& Charette, 2000). Removal of researcher interaction was achieved by allowing the software to automatically progress 
from one parameter run to the next, while carrying forward the best parameter values (Rybchynski, 2005).

\section{Learning rate (lr)}

While attempting to minimize the average squared error of the network's output, the learning rate determines the step size and thus the speed with which the network achieves its minimum in the criterion function, resulting in convergence (Witten \& Frank, 2005). If the learning rate is too high and the error function has several minima, the search can overshoot and miss the minimum entirely, or it can result in oscillations. On the contrary, if the learning rate is too small, convergence will eventually happen, but may take a very long time; this is the main reason backpropagation networks are slow (Masters, 1993). In the search for the minimum, MIRG's ANN RFW also uses the learning rate increment $\left(\mathrm{l} \mathrm{r}_{-} \mathrm{inc}\right)$ and learning rate decrement $\left(\mathrm{l} \_\mathrm{dec}\right)$ values as the driving parameters.

\section{Momentum}

In order to enhance the performance of the network and speed up the learning process, the momentum term is introduced in the update of network weights. The term adds a proportion of the update value from the previous iteration to the new weight change and as such "smooths the search process by making changes in direction less abrupt" (Witten \& Frank, 2005). This in turn helps ensure that the learning algorithm does not get caught up in a local minimum (Masters, 1993). 


\section{Weight-decay constant $(\lambda) \&$ Weight-elimination scale factor $\left(w_{0}\right)$}

The purpose of the weight-decay constant is to limit the influence of nonmeaningful connections on the network. The constant penalizes large weights by preventing them from increasing since they might not contribute a correspondingly large reduction in the error (Witten \& Frank, 2005). Depending on the network performance, the ANN RFW uses weight-decay constant increment $\left(\lambda \_\right.$inc $)$or decrement $\left(\lambda \_\right.$dec $)$to penalize connections.

Like the weight-decay constant, the weight-elimination scale factor is used for network pruning and regularization of the network training, i.e. minimize overfitting to the noise on the training data (Bishop, 1995). The weight-elimination scale factor sets the scale for the 'large' and 'small' weights. "When $w_{o}$ is small, the small weights will be forced to zero resulting in a fewer large weights (i.e. weight-elimination). A large $w_{o}$ causes many small weights to remain and limits the size of large weights (i.e. weightdecay)" (Ennett, 2003).

Combining the effects of the weight-decay constant and the weight weightelimination scale factor with the mean squared error function, the weight-elimination cost function is obtained (Weigend, Rumelhart, \& Huberman, 1990), (Ennett \& Frize, 2003).

$$
E(W)=E_{0}(W)+\lambda \times \sum_{i j} \frac{\frac{w_{i j}^{2}}{w_{0}^{2}}}{1+\frac{w_{i j}^{2}}{w_{0}^{2}}}
$$

where,

$E_{0}=\operatorname{SSE}$ (sum squared error).

$\lambda=$ weight-decay constant

$w_{i j}=$ weight of connecting node $\mathrm{i}$ and $\mathrm{j}$.

$w_{0}=$ weight elimination scale factor 


\section{Error ratio (err_ratio)}

User-defined value, error ratio is used to "measure the change from the current to previous error to determine how the learning rate, weight decay constant and momentum will be adapted to improve the network performance" (Ennett, 2003). With improvement in performance of the network, error ratio should decrease.

\subsubsection{Network structure}

Medical data is generally not linear, and is not easily mathematically characterized. As such, linear neural networks are not the best for modeling the medical domain; hence non-linear networks with one or more hidden layers are a preferred choice. The literature indicates that there is no reason why more than one hidden layer should be used for ANNs, as it does not add more knowledge to the network (Hertz et al., 1991), (Masters, 1993), (Ennett, 2003). In addition, multiple hidden layers slow training

dramatically because of error backpropagation and constant changes to the gradient and hence the weights (Masters, 1993).

Finding the optimal number of hidden nodes in a hidden layer is a brute force and a time-consuming task. The use of too many hidden nodes not only increases the training time, but it can potentially lead to overfitting as the network learns "insignificant aspects of the training set, aspects that are irrelevant to the general population" (Masters, 1993). On the contrary, too few hidden nodes might be insufficient for learning and solving the problem. While Hertz et. al. (1991) state that the necessary number of hidden 'units' is not known in general, and may grow exponentially with the number of input units, Masters (1993) states that the rough guideline for choosing the number lies in the geometric pyramid rule: 


$$
\text { hidden nodes }=\sqrt{\text { TotalOutputNodes } * \text { TotalInputNodes }}
$$

Masters (1993) also reiterates that this number should only be used as a rough guideline and might not be considered sufficient for networks with a smaller number of input and output variables. Two other guidelines are considered for determining the number of hidden nodes: Livingstone's and Manallack's method (Livingstone \& Manallack, 1993) as well as Kolmogorov's Theorem (Bishop, 1995), equations 2.11 and 2.12 respectively.

$$
\text { hidden nodes }=\frac{\text { TotalTrainingCases } * \text { TotalOutputNodes }}{\text { TotalNetworkWeights }}
$$
where,

hidden nodes $>3$ to ensure good generalization

Livingston's and Manallack's method produces a large number of hidden nodes, and for a database the size of the CNN's, it is not considered a viable solution with regards to determining the network structure. Past MIRG researchers have also found that the neural networks using the CNN database did not require as many hidden nodes as Livingstone and Manallack suggest (Khan, 2006).

$$
\text { hidden nodes }=2 n+1 \text {, where } n=\# \text { of input variables }
$$

Kolmogorov's Theorem, as adapted to neural networks, states that mapping $y(x)$ from $n$ input variables $x_{i}$ to the output variable $y$ can be represented exactly by a three-layer neural network having $(2 n+1)$ units in the hidden layer (Bishop, 1995). MIRG's ANN RFW tool is setup to use a modified version of Kolmogorov's Theorem to determine the max number of hidden nodes. ANN RFW attempts classification on structures with the number of hidden nodes ranging from 0 to $2 n+1$, after which the structure with the best 
criteria is chosen as the model. As variables are removed, the number of hidden nodes decreases, which in turn reduces the network complexity and computational time.

\subsubsection{Support Vector Machines}

Support Vector Machines (SVMs) are a set of supervised learning methods that can be used for both classification and regression problems (Vapnik, 1995). Developed by Vapnik (1995), SVM goal is to implement principles of statistical "learning theory that addresses the fundamental issue of how to control the generalization ability" and produce input-output mapping of data instances (Haykin, 1999). Consider a set of empirical data:

$$
\left(x_{1}, y_{1}\right), \ldots,\left(x_{m}, y_{m}\right) \in \mathfrak{R} \times \mathrm{Y}, \mathrm{Y}=\{-1,1\}
$$

where,

$$
\begin{aligned}
& i=1 \ldots m \\
& x_{i}=\text { patterns (input data) } \\
& y_{i}=\text { labels or targets (output) }
\end{aligned}
$$

Vapnik (1995) introduced the concept of the N-dimensional hyperplane used to separate instances of two different classes, $\mathrm{Y}=\{-1,1\}$. The hyperplane is bound by a margin, which is defined as "the minimal distance of an example to a decision surface" leading to the idea that the "risk is minimized when the margin is maximized" (Maimon \& Rokach, 2005). The subset of training examples that constrain the width of the margin and the optimal hyperplane are known as support vectors (Vapnik, 1995). In the simplest of hyperplane classifications, a 2-dimensional model (figure 2.6), the class of hyperplanes for linearly separable patterns takes the form (Chen et al., 2005):

$$
(\mathbf{w} \cdot \mathbf{x})+b=0 \quad \mathbf{w} \in \mathfrak{R}^{\mathbf{N}}, b \in \mathfrak{R}
$$


where,

$$
\begin{aligned}
& \mathbf{w}=\text { adjustable weight vector } \\
& \mathrm{x}=\text { input vector } \\
& b=\text { bias }
\end{aligned}
$$

This class of hyperplanes correspond to decision functions:

$$
f(x)=\operatorname{sign}((\mathbf{w} \cdot \mathbf{x})+b)
$$

For two samples, $\mathrm{x}_{1}$ and $\mathrm{x}_{2}$, belonging to a different class such that $\left(\mathbf{w} \cdot \mathrm{x}_{1}\right)+b=1$ and $\left(\mathbf{w} \cdot \mathrm{x}_{2}\right)+b=-1$, respectively, the distance of $\mathrm{x}_{1}$ and $\mathrm{x}_{2}$, measured perpendicularly to the hyperplane, gives the margin of:

$$
\frac{\mathrm{w}}{\|\mathrm{w}\|} \cdot\left(\mathrm{x}_{1}-\mathrm{x}_{2}\right)=\frac{2}{\|\mathrm{w}\|}
$$

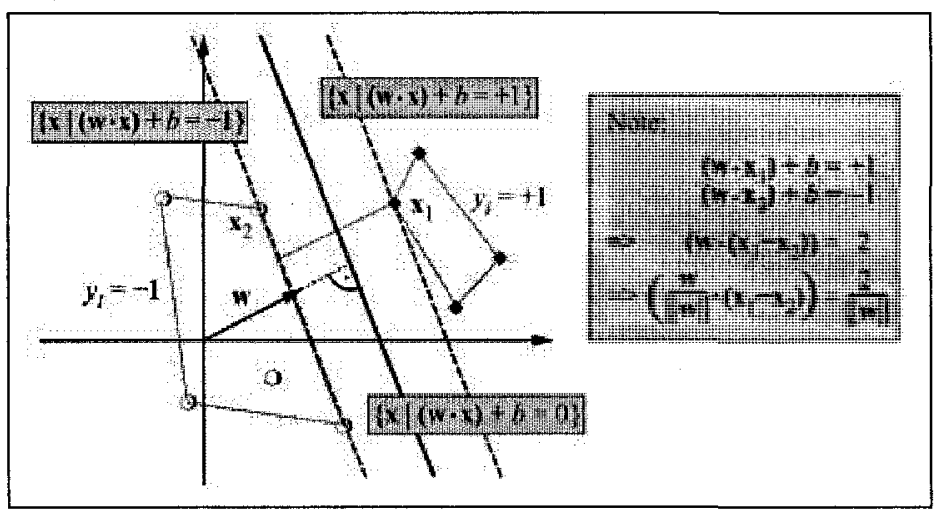

Figure 2.6: The optimal hyperplane for a linear 2-class classifier, represented by circles and diamonds (Chen et al., 2005).

Although there may be several hyperplanes separating the two classes of data, the unique hyperplane defining the maximum margin of separation for the two classes is defined as:

$$
\max _{w, b} \min \left\{\left\|\mathbf{x}-\mathbf{x}_{i}\right\|: \mathbf{x} \in \mathfrak{R}^{\mathrm{N}},(\mathbf{w} \cdot \mathbf{x})+b=0, i=1, \ldots, m\right\}
$$

The optimal hyperplane is achieved by solving the optimization problem:

$$
\min _{w, b} \frac{1}{2}\|w\|^{2}
$$




$$
\text { subject to : } \left.\quad y_{i}\left(\left(\mathbf{w} \cdot \mathrm{x}_{i}\right)\right)+b\right) \geq 1, \text { for } i=1, \ldots, n
$$

Beyond the scope of this research, the optimization is solved by introducing the Lagrange multipliers $\alpha_{i} \geq 0$ and the Lagrange function:

$$
L(\mathbf{w}, b, \alpha)=\frac{1}{2}\|\mathrm{w}\|^{2}-\sum_{\mathrm{i}=1}^{\mathrm{n}} \alpha_{i}\left(y_{i}\left(\left(\mathbf{w} \cdot \Phi\left(\mathrm{x}_{\mathrm{i}}\right)\right)+b\right)-1\right)
$$

$L$ is maximized with respect to primal variables $\{\mathbf{w}, b\}$ and minimized with respect to dual variables $\alpha_{i}$. By finding the derivates of $L$ with respect to the $\mathbf{w}$ and $b$, the primal variables can be replaced by:

$$
\sum_{\mathrm{i}=1}^{\mathrm{n}} \alpha_{\mathrm{i}} \mathrm{y}_{\mathrm{i}}=0 \quad \text { and } \quad \mathbf{w}=\sum_{i=1}^{n} \alpha_{i} y_{i} x_{i}=0
$$

Furthermore, the support vector correspond to non-zero $\alpha_{i}$, which by the Karush-KuhnTucker (KKT) complimentary conditions of optimization means that all other constrains in equation 2.17 must be zero (Maimon \& Rokach, 2005):

$$
\alpha_{i}\left(\mathrm{y}_{\mathrm{i}}\left(\left(\mathbf{w} \cdot \mathrm{x}_{i}\right)\right)+b-1\right)=0, \quad \text { for } i=1, \ldots, n
$$

The original hyperplane decision function equation 2.12 can then be written as:

$$
f(\mathrm{x})=\operatorname{sign}\left(\sum_{\mathrm{i}=1}^{\mathrm{n}} \alpha_{i} y_{i}\left(\mathrm{x}^{\bullet} \mathrm{x}_{i}\right)+\mathrm{b}\right)
$$

\subsubsection{Kernel functions}

By far the simplest way to divide two classes is to use a linear classifier, as described in the previous section. However, linear classifier functions often have a limited functionality as data is not always linearly separable, resulting in a classifier under fitting the data. To deal with this predicament, SVM makes use of kernel functions

which map the data into a feature space where maximum-margin hyperplanes can be 
constructed (Maimon \& Rokach, 2005). According to Cortes and Vapnik (1995), "to construct a hyperplane in a feature space one first has to transform the $n$-dimensional input vector $\boldsymbol{x}$ into an $\mathrm{N}$-dimensional feature vector through a choice of an $\mathrm{N}$-dimensional vector function $\Phi: \mathfrak{R}^{n} \rightarrow \mathfrak{R}^{N}$." The N-dimensional linear weight vector $\mathbf{w}$ and bias $b$ is constructed for the set of transformed vectors $\Phi\left(\mathrm{x}_{i}\right)=\Phi_{1}\left(\mathrm{x}_{i}\right), \Phi_{2}\left(\mathrm{x}_{i}\right), \ldots, \Phi_{\mathrm{N}}\left(\mathrm{x}_{i}\right)$ (for $i=1, \ldots, 1)$ as a modified equation 2.12 (Cortes \& Vapnik, 1995):

$$
f(\mathrm{x})=\mathrm{w} \cdot \Phi(\mathrm{x})+\mathrm{b}
$$

Determining the best kernel function is the subject of active research, as different functions give similar classification accuracy and sets of support vectors (Vapnik, 1998), (Schölkopf \& Smola, 2002). The most commonly used kernel functions, as summarized in table 2.7 are Polynomial (degree $d$ ), Radial Basis Function (RBF), and sigmoid (Schölkopf \& Smola, 2002).

Table 2.7: Common SVM kernel functions

\begin{tabular}{ll}
\hline Kernel function & $\mathbf{K}\left(\mathbf{x}, \mathbf{x}_{i}\right)$ \\
\hline Radial Basis Function & $\exp \left(-\gamma\left\|\mathrm{x}-\mathrm{x}_{\mathrm{i}}\right\|^{2}\right), \gamma>0$ \\
Polynomial (degree $d)$ & $\left(\left(\mathrm{x}^{\mathrm{T}} \mathrm{x}_{\mathrm{i}}\right)+\eta\right)^{d}$ \\
Sigmoid & $\tanh \left(\gamma\left(\mathrm{x}^{\mathrm{T}} \mathrm{x}_{\mathrm{i}}\right)+\eta\right), \gamma>0$ \\
\hline
\end{tabular}

Literature review suggests that RBF is a 'reasonable first choice' with respect to SVM kernels and classification (Maimon \& Rokach, 2005), (Chen et al., 2005), (Schölkopf \& Smola, 2002). As such, it was the kernel of choice in this research. Incorporating the kernel function with equation 2.21 , by replacing the dot product of $\left(\mathrm{x}^{\bullet} \mathrm{x}_{i}\right)$, the following hyperplane decision function is obtained:

$$
f(\mathrm{x})=\operatorname{sign}\left(\sum_{\mathrm{i}=1}^{\mathrm{n}} \alpha_{i} y_{i} K\left(\mathrm{x}, \mathrm{x}_{i}\right)+\mathrm{b}\right)
$$


The optimization problem is then:

$$
\max _{a} \sum_{\mathrm{i}=1}^{\mathrm{n}} \alpha_{\mathrm{i}}-\frac{1}{2} \sum_{\mathrm{i}, \mathrm{j}=1}^{\mathrm{n}} \alpha_{i} \alpha_{j} y_{y_{j}} K\left(\mathrm{x}, \mathrm{x}_{i}\right)
$$

subject to: $\quad \alpha_{i} \geq 0, \quad i=1, \ldots n, \quad \sum_{\mathrm{i}=1}^{\mathrm{n}} \alpha_{\mathrm{i}} \mathrm{y}_{\mathrm{i}}=0$

\subsubsection{Soft Margin Support Vector Classifier: C-SVC}

An ideal SVM classifier introduces a hyperplane that fully separates two classes of data. Nonetheless, this is often impossible as data samples frequently 'violate' constraints of the optimal hyperplane. To allow flexibility for such issues, Cortes and Vapnik (1995) introduced a slack variable $\xi_{i}$ (modification to equation 2.17) whose purpose is to 'relax' the margin constraints:

$$
y_{i}\left(\left(\mathbf{w} \cdot \Phi\left(\mathrm{x}_{\mathrm{i}}\right)\right)+b\right) \geq 1-\xi_{i}, \quad \xi_{i} \geq 0, i=1, \ldots, n
$$

By controlling the classifier capacity $(\|\mathbf{w}\|)$ and the sum of the slack variables $\xi_{i}$, the soft margin classifier minimizes the following objective function (Maimon \& Rokach, 2005):

$$
\min _{\mathrm{w}, \mathrm{b}, \xi} \frac{1}{2}\|\mathrm{w}\|^{2}-\mathrm{C} \sum_{\mathrm{i}=1}^{\mathrm{n}} \xi_{i}, \quad \text { where } C>0
$$

The regularization constant, $C$, is used to control the trade-off between the training errors and rigid margins, by permitting some misclassifications (Vapnik, 1995), (Schölkopf \& Smola, 2002). Because of the high level of non-linearity in medical data, this thesis uses the C-SVM classifier (C-SVC). Lastly, incorporating $C$ with the Lagrange multipliers from equation 2.24 and 2.25, the Lagrange multiplier $\alpha_{i}$ is subject to $0 \leq \alpha_{i} \leq C, i=1, \ldots . n$,

$$
\sum_{i=1}^{n} \alpha_{i} y_{i}=0
$$




\subsection{Data preparation}

Medical data is unique and requires consideration of the following heterogeneity issues before it is used (Cios \& Moore, 2002):

1. Volume and complexity

2. Physician's interpretation

3. Sensitivity and specificity analysis

4. Poor mathematical characterization

5. Canonical form (same concept represented with different terminology)

Consistency in data collection is necessary to minimize the impact of the above issues and in order to ensure good quality data is used in research. MIRG research is conducted on medical data collected by the Canadian Neonatal Intensive Care Unit Network (CNN), which includes 17 tertiary care NICUs, or approximately $80 \%$ of the tertiary care NICU beds in Canada (McMillan, Chan, \& Lee, 1997). The data collection was funded by the Medical Research Council of Canada, and was done between January $8^{\text {th }}, 1996$ and October $31^{\text {st }}, 1997$. It includes 20,488 baby admissions. Data was collected for day 1 (admission day), day 3,14 , and 28 or discharge. However, issues of missing values, data imbalance with respect to a priori levels and data dimensionality reduction all have to be addressed by the users of this database before commencing data mining.

\subsubsection{Missing values}

Missing values and class imbalance are challenges for data mining techniques. Data that medical professionals consider irrelevant for diagnosis or that is not recorded because a medical test was not performed, are considered 'do not care' conditions. On the contrary, values that were misplaced, forgotten or mistakenly erased are considered lost values. Regardless of type, both 'do not care' and 'lost' data are considered 
important to medical data mining as each patient case offers valuable knowledge. The CNN database used by MIRG is no exception to the issue of missing values and incomplete data sets. Literature further classifies methods to handle this issue as sequential (or pre-processing) methods and parallel methods (Maimon \& Rokach, 2005). Unlike the sequential methods, parallel methods take into account the attributes with missing values during the main process of acquiring knowledge by using rule induction (for example decision trees). However, ANN and SVM cannot perform well on databases with missing values (Ennett, 2003); hence for this thesis, parallel methods are not considered a valid solution for the missing value problem.

Instead, MIRG uses sequential methods to deal with missing values in the CNN database. While deleting cases with missing attribute values, method known as listwise/ casewise deletion or complete case analysis, is considered legitimate, the process itself strips the database of valuable information (Ennett, 2003), (Maimon \& Rokach, 2005). With respect to the $\mathrm{CNN}$ database, cases that have the least amount of missing values are those of the sickest infants, and removing less sick babies would result in a model that is not representative of the entire population (Ennett, Frize, \& Walker, 2001). Several of the MIRG researchers have worked on validating methods for imputation of missing values in the CNN database, which include the following:

\section{Statistical imputation}

- Missing values were replaced with mean and median values for the particular variable, for the outcome of mortality. However, the database was found to be biased towards the survivors and non-survivors were not classified correctly (Tong, 2000). 


\section{Normal distribution replacement}

- Missing values were replaced by a random value from the normal distribution.

\section{Case-Based Reasoner (CBR)}

- This knowledge base system uses a retrieval algorithm (k-Nearest Neighbour) to determine the most similar cases in the case base (Ennett, 2003). By plotting the data points in an n-dimensional space, the k-NN calculates the weighted distance between the cases in the repository to that of the target. The mean of the 10 closest matching cases are used to impute the value of the missing variable. Ennett (2003) validated the imputation of missing values using linear ANN weights for the day 1 data with missing values, while Khan (2006) performed the imputations using both linear and non-linear ANN weights on day 1 and day $3 \mathrm{CNN}$ data. The imputations were done using variable weights biased towards the output of mortality, as determined by: a) a physician domain expert, and b) linear and non-linear neural networks (Ennett, 2003), (Khan, 2006). Townsend (2007) performed the imputation of missing values for the day 1 data as well; however, the imputations were done by using uniform weights for all variables as opposed to the weights determined by the ANNs or assigned by physicians. Townsend (2007) reasoned that this was to avoid a bias towards a particular outcome, such as mortality, as done in previous work.

\subsubsection{CBR uniform weight imputation}

The original CNN database used in this thesis contains 20488 patient files. Prior to proceeding with model development, the database had to be screened and analyzed. 
Transfer of babies between hospital sites and re-admissions resulted in duplicate cases of patient files. After removal of duplicate files, cases with extreme outliers, missing outcome information (originally mortality), birth weight and variable 'small for gestational age' the total number of cases included 19814 babies (Townsend, 2007). The summary of missing value statistics for the 30 SNAP variables in the CNN Database is seen in table 2.8 .

Table 2.8: CNN (19814 cases) database missing value statistics (Townsend, 2007)

\begin{tabular}{llc}
\hline Parameter description & Variable & Percent missing \\
\hline Small for gestational age & SGA & 0.00 \\
Birth weight & bthwght & 0.00 \\
Presence of apnea & apnea & 0.02 \\
Stool guaiac & guaiac & 0.02 \\
Presence of seizures & seizure & 0.03 \\
Highest heart rate & hheartr & 0.22 \\
Lowest heart rate & lheartr & 0.26 \\
Highest respiratory rate & hrespr & 0.36 \\
Lowest temperature & ltempf & 0.41 \\
Apgar score at 5 minutes & Apgar5 & 1.73 \\
Highest glucose & hgluc & 8.72 \\
Highest mean blood pressure & hbloodp & 12.70 \\
Lowest glucose & lgluc & 14.51 \\
Lowest mean blood pressure & lbloodp & 16.07 \\
Lowest white blood cell count & LWBC & 17.42 \\
Highest hematocrit & hhema & 18.56 \\
Lowest platelet count & lplt & 28.55 \\
Lowest absolute neutrophil count & LANC & 28.57 \\
Lowest hematocrit & lhema & 33.53 \\
Highest immature/total neutrophil ratio & hitnrat & 35.77 \\
Lowest serum pH & lserum & 39.85 \\
Highest pCO2 & hpco2 & 40.26 \\
Lowest urine output & lurine & 52.68 \\
Highest sodium & hsodium & 59.53 \\
Highest potassium & hpotass & 62.65 \\
Lowest pO2 & lpo2po2 & 63.14 \\
Lowest pO2/FiO2 ratio & Po2fio2r & 64.11 \\
Lowest sodium & lsodium & 66.00 \\
Lowest potassium & lpotass & 68.04 \\
Highest oxygenation index & OI & 72.76 \\
\hline & & \\
& &
\end{tabular}

The next step was to deal with missing values present in the database by imputing data in order to ensure sufficient number of cases are present for training and testing of the learning algorithms. Prior to imputation and upon feedback from the collaborating 
CHEO neonatologist, Dr. Erika Bariciak (2006), variables 'stool guaiac', 'highest potassium' and 'lowest potassium' were removed from the database as they are normally not measured within the first 12 hours of admission. Missing values for the remaining 27 day 1 variables used in this thesis were next imputed by Townsend (2007) using uniform weights. In order to minimize the error from imputation of missing values, Townsend (2007) imputed up to 8 missing variables per case (i.e. up to $30 \%$ of a particular case is potentially imputed). Patient cases with more than 8 missing variables were discarded, resulting in the final database of 13584 complete cases after imputation. Table 2.9 summarizes pre-imputation variable statistics for the 13584 cases that qualified for imputation of missing values.

Table 2.9: Distribution of missing values in the 13584 CNN database (Townsend, 2007)

\begin{tabular}{lrrrrrr}
\hline Variable & Min & Max & Mean & St. Dev. & \# Missing & \% Missing \\
\hline SGA & 0 & 1 & 0.04 & 0.20 & 0 & 0.00 \\
birth weight & 280 & 6320 & 2338.65 & 1042.19 & 0 & 0.00 \\
apnea & 1 & 4 & 1.17 & 0.56 & 0 & 0.00 \\
seizure & 1 & 3 & 1.05 & 0.29 & 0 & 0.00 \\
hheartr & 60 & 327 & 161.84 & 18.00 & 1 & 0.01 \\
lheartr & 0 & 197 & 126.73 & 15.52 & 3 & 0.02 \\
ltempf & 68 & 113 & 97.43 & 1.21 & 3 & 0.02 \\
hrespr & 0 & 180 & 58.61 & 29.06 & 18 & 0.13 \\
Apgar5 & 0 & 10 & 7.83 & 1.74 & 33 & 0.24 \\
hbloodp & 0 & 121 & 49.07 & 11.31 & 260 & 1.91 \\
hgluc & 0.9 & 65.3 & 5.44 & 2.59 & 341 & 2.51 \\
lbloodp & 0 & 100 & 36.71 & 9.54 & 392 & 2.89 \\
LWBC & 0.39 & 1442 & 15.17 & 22.37 & 483 & 3.56 \\
hhema & 0 & 82 & 49.37 & 8.84 & 624 & 4.59 \\
lgluc & 0 & 20.7 & 3.42 & 1.63 & 988 & 7.27 \\
lplt & 0.22 & 3337 & 212.63 & 102.80 & 1594 & 11.73 \\
LANC & 0 & 686000 & 8599.17 & 12648.83 & 1888 & 13.90 \\
lserum & 4.4 & 7.79 & 7.31 & 0.11 & 2535 & 18.66 \\
hpco2 & 4.44 & 172 & 46.80 & 14.06 & 2600 & 19.14 \\
lhema & 0 & 80 & 48.45 & 9.23 & 2796 & 20.58 \\
hitnrat & 0.00 & 1 & 0.16 & 0.16 & 2999 & 22.08 \\
lurine & 0 & 11.73 & 0.89 & 0.82 & 5471 & 40.28 \\
hsodium & 106 & 182 & 136.20 & 5.06 & 6395 & 47.08 \\
lpo2po2 & 0 & 434 & 61.09 & 33.47 & 6476 & 47.67 \\
Po2fio2r & 0 & 12.42 & 1.90 & 1.27 & 6642 & 48.90 \\
lsodium & 86 & 180 & 135.51 & 5.19 & 7392 & 54.42 \\
OI & 0 & 275 & 9.10 & 16.13 & 8297 & 61.08 \\
\hline & & & & & & \\
\hline
\end{tabular}


Majority of the variables used had less than $25 \%$ missing values. Variables with a large percent of missing values (ex. OI, lsodium) were kept because they were deemed important, and due to the reasonable standard deviation (Bariciak, 2006). As mentioned in section 2.4.1, the imputations were done using the k-NN algorithm, thus only the 10 closest matching cases were used to impute the value of the missing variable. Once the imputations were complete, the 13584 database then had to be processed, adjusted and analyzed with respect to NEC. This is discussed in the methodology section.

\subsubsection{Data imbalance}

In addition to the issue of missing values, data mining also has to consider data imbalance. Rare cases are of most interest to, as the goal is to uncover patterns that are hidden in vast amounts of data (Maimon \& Rokach, 2005). With respect to neural network training, "if some classes have much fewer samples, i.e. a priori level, compared to other classes, the neural network system may respond wrongly for minority classes because the overwhelming samples in the majority classes dominate the adjustment procedure in training" (Wang \& Xiuju, 2005). This in turn makes generalization difficult (Maimon \& Rokach, 2005). Obtaining more medical data to increase the a priori of the rare cases is not an option, so the data imbalance must be dealt with in other ways. While it is possible to randomly remove some of the majority cases (random undersampling method), this solution is not considered beneficial as the entire population is not correctly represented. Removal of useful data is risked when cases are removed due to missing values as well as undersampling (Ennett, 2003). MIRG instead resorts to boosting the numbers of minority cases by random oversampling, and does so for the rare cases of the training data sets. Ennett (1999) performed several ANN trials to ensure that 
oversampling the rare cases does not lead to data overfitting, and found that resampling the rare case up to $20 \%$ for training was optimal for training.

\subsubsection{Data dimensionality reduction (DDR)}

Medical databases have a large amount of data and variables. Although it is beneficial to have as much information as possible, the high-dimensionality of data makes it difficult to analyze and model due to irrelevant and redundant information that often interferes with useful values and attributes (Wang \& Xiuju, 2005). As Wang and Xiuju (2005) suggest, "the problem of DDR is to select a subset of attributes which represent the concept of data without losing important information". The attribute space search is performed in one of two ways: forward selection or backward elimination (Witten \& Frank, 2005). Forward selection requires a use of a predetermined attribute subset, as deemed important by the domain expert. This attribute set is not the minimum data set; instead it is used as a starting point to which additional attributes are added one by one. For example, the 9 SNAPPE-II variables can be considered a subset to which additional attributes are added in order to expand the attribute set and improve performance. On the contrary, backwards elimination starts off with the entire attribute set, for example all of the SNAP variables, from which attributes are removed one-byone, or several at a time. There are tradeoffs for either method: forward selection requires a domain knowledge and thus might be biased, whereas backwards elimination can be extremely inefficient for working with large-scale databases with many variables (Maimon \& Rokach, 2005). MIRG uses backwards elimination, and in previous research has created minimum data sets for various outcomes and complications: mortality, length of stay, ventilation, preterm birth, BPD, and IVH. 
Furthermore in order to evaluate the importance of each variable, ANNs use the relative weight reduction as modified by Rybchynski (2005).

\subsubsection{Relative Weight Reduction Method (ANN)}

With the introduction of non-linear network to the ANN RFW, Rybchynski (2005) investigated weight reduction methods necessary for the data dimensionality reduction and creation of a minimum data set. For a linear network, weight extraction is a fairly straightforward process: scale the magnitude of the connection weights between the input and output nodes between 0 and 100, and remove the input nodes with the smallest weights (Frize, Ennett, \& Hebert, 2001), (Rybchynski, 2005). The network is retrained again, and the process is repeated. For a non-linear network, the introduction of hidden layers complicates this calculation as it requires a series of computations that take into account the hidden layer and the nodes associated with it, and their relationship to the input and output of the network. Rybchynski adopted a weight reduction method developed by Garson and modified by Goh (1995), and modified it so as to "integrate the hidden-output weights into the algorithm while maintaining the basic approach of Garson-Goh's relative weight calculation" (Rybchynski, 2005). The equations associated with the modifications to Garson-Goh are the following (Rybchynski, 2005):

1) Calculation of the relative weight of the hidden-output connections into the output node $(k=1)$ :

$$
r w_{j k}=\frac{a b s\left(w_{j k}\right)}{\sum_{j} a b s\left(w_{j k}\right)}
$$


2) Calculation of the relative weight of the input-hidden connections into each hidden node (for each hidden node $j$ ):

$$
r w_{i j}=\frac{a b s\left(w_{i j}\right)}{\sum_{i} a b s\left(w_{i j}\right)}
$$

3) Multiplication of each relative weight input-hidden connection value by the relative weight value of its connected hidden-output weight (for each input $i$ ):

$$
\mathrm{P}_{\mathrm{ik}}=\mathrm{rw}_{\mathrm{ij}} * \mathrm{rw}_{\mathrm{jk}}
$$

4) Summation of the final relative weight products (for each input i):

$$
\mathrm{S}_{\mathrm{i}}=\sum_{\mathrm{i}} \mathrm{P}_{\mathrm{ik}}
$$

5) Division of the sum of the final relative weights for each input node by the total of all relative weights (for each input i):

$$
R I_{i}=\frac{S_{i}}{\sum_{i} S_{i}}
$$

where,

$i$ : \# of input nodes

$j:$ \# of hidden nodes

$k$ : \# of output nodes ( $k=1$ for MIRG research)

$w_{i j}$ : connection weight between input node $i$ and hidden node $j$

$w_{j k}$ : connection weight between hidden node $j$ and output node $k$

$r w_{i j}$ : relative connection weight between input node $i$ and hidden node $j$

$r w_{j k}$ : relative connection weight between hidden node $j$ and output node $k$

$R I_{i}:$ Relative Importance of input node $i$ 


\section{METHODOLOGY}

Several factors are considered in the development of the NEC outcome prediction model(s) and are based on the following findings in the medical literature:

1. Can scores for predicting mortality be used as markers of disease severity for other outcomes, such as NEC (Dorling \& Field, 2008)?

2. NEC is a multivariable complication with an unclear pathogenesis.

3. Clinical characteristics and laboratory features are believed to be different for neonatal population of infants born preterm and those born at term.

In response to these issues, three NEC models are explored in this thesis:

1. NEC model for the total population

a. model with gestation as a binary variable

$($ GestAge $<33$ weeks $=1$, GestAge $\geq 33$ weeks $=-1)$

b. model with normalized gestation age (between -1 and 1)

2. NEC in very low birth weight (VLBW) population

3. NEC in population with gestation $<33$ weeks

Model development, with the goal of achieving a minimum data set for NEC outcome prediction, is attempted with MIRG's ANN RFW, and the Library for Support Vector Machines (LIBSVM C-SVC v.2.86) tool developed by Chang and Lin (2001). ANN RFW tool has been used by MIRG for development of mortality, length of stay, ventilation, BPD and IVH scoring models. The SVM classification has to date been used by MIRG only for segmentation and extraction of regions of interest in clinical thermal 
infrared images (Herry, 2008). As such the methodology described here with respect to the LIBSVM C-SVC tool is new to MIRG.

\subsection{Data Understanding and Preparation}

After the imputation of missing values was completed by Townsend (2007), the 13584 case CNN database was further modified for NEC. Outcome information for NEC was added to the 13584 cases, as were the variables gestational age and neonate gender, which were not considered important for Townsend's analysis, but are relevant in NEC outcome (Bariciak, 2006). Dr. Bariciak (2006) also suggested that high blood pressure be removed from NEC analysis as it is not relevant to the outcome itself, as well as the SGA variable since it is derived from the birth weight and gestational age variables. Lastly, any of the 13584 cases with missing NEC outcome, and missing information/ambiguity for the neonate gender were also removed at this point. The resulting NEC database had 13133 patient cases with 27 variables, as summarized in table 3.1. With respect to Ennett's (2001) conclusion that "patients who have fewer missing values have a higher mortality rate than patients with more missing values", this was found to be true for NEC as well. The original CNN database (20488 cases) has a total of 344 NEC patients whereas the processed and final database has 312 .

Table 3.1: Day 1 variables used in NEC modeling

\begin{tabular}{lll}
\hline & Variable & Variable Description \\
\hline 1 & CASELINK & Patient ID - administrative variable \\
$\mathbf{2}$ & LURINE & Lowest urine output \\
3 & HRESPR & Highest respiratory rate \\
4 & LPLT & Lowest platelet count \\
$\mathbf{5}$ & $\mathbf{S G A}$ & Small for gestational age (SNAPPE-II model only) \\
\hline
\end{tabular}




\begin{tabular}{lll}
\hline 6 & HGLUC & Highest glucose \\
7 & LHEMA & Lowest hematocrit (Hct, Ht) \\
$\mathbf{8}$ & APGAR5 & Apgar score @ 5min \\
$\mathbf{9}$ & LBLOODP & Lowest mean blood pressure \\
10 & HHEMA & Highest hematocrit \\
11 & LGLUC & Highest glucose \\
$\mathbf{1 2}$ & BTHWT & Birth weight \\
13 & HSODIUM & Highest sodium \\
14 & HITNRAT & Highest immature/total neutrophil ratio \\
15 & LWBC & Lowest white blood count \\
16 & LANC & Lowest absolute neutrophil count \\
17 & LHEARTR & Lowest heart rate \\
18 & HHEARTR & Hearth rate high \\
19 & OI & Oxygenation index \\
20 & APNEA & Presence of apnea \\
$\mathbf{2 1}$ & LTEMPF & Lowest temperature \\
22 & SEIZURE & Presence of seizure \\
23 & LSODIUM & Lowest sodium \\
24 & LPO2PO2 & Lowest pO2 \\
25 & LSERUM & Lowest pH \\
26 & PO2FIO2R & Lowest pO2/FiO2 ratio \\
27 & HPCO2 & Highest pCO2 recorded \\
28 & GEST & Gestational age \\
29 & SEX & Baby's gender \\
30 & NEC & Outcome variable \\
\hline
\end{tabular}

** NOTE: SNAPPE-II variables are highlighted in bold

To understand the complex interaction of the variables used in modeling NEC, Statistical Package for Social Sciences (SPSS) is used to determine the correlation between input and output variables. The correlation used, Spearman Rank Correlation (rho, $\rho$ ), is performed to test the strength of the relationships between any two variables. Spearman correlation is used instead of the Pearson correlation for the reason that: 1) it does not require linear relationship assumptions between variables, 2) it can be used for ordinal and nominal data, and 3) and it does not require knowledge about the variable frequency distribution (SPSS 16.0 for Windows, 2007). To assess the statistical significance of $\rho$ between any two variables, the 2-tailed significance test is performed in 
SPSS. The probability value $p<0.05$ from the 2 -tailed significance test is taken to imply statistical significance. Ideally, the minimum data set would not be made up of variables that are significantly correlated, i.e. the variables are independent.

\subsubsection{ANN RFW vs. C-SVC datasets for outcome prediction}

MIRG's ANN RFW uses two datasets for training: the training set and the test set. The training set is used by the network to 'learn' and obtain the best parameters and structure with respect to network weights and biases. The test set evaluates the performance of the network after it has been trained. Optimal network parameters found during training and testing are validated with a third dataset - a verification dataset. Emphasizing that "in many respects, proper validation is more important than proper training", Masters (1993) states that it is imperative that no part of the verification data is used as training. Using the same data for training and validation "biases the indication of what can be expected of the network when it is used in general population" (Masters, 1993). A large difference in training versus validation results can indicate that the overtraining has occurred and that the network does not generalize well. Rybchynski (2005) incorporated ten verification sets as part of the ANN RFW validation.

The Library for Support Vector Machines (LIBSVM) C-SVC v2.86 tool by Chang and Lin (2001), makes use of the cross-validation method for training the classifier, and thus integrates the training and test sets as one dataset. The leave-one-out or $n$-fold cross-validation, performed in this research with the LIBSVM C-SVC tool is the following:

1. A training set is divided into $n$ subsets of equal size, where $n=5$. 
2. The classifier is trained $n$ times, where on the $i$-th iteration $(i=1, \ldots, n)$, the classifier is trained on all subsets except the $i$-th one.

A separate dataset is then used for verification, just as is done with the ANN RFW tool. The difference in training methods is taken into account when developing datasets for ANN RFW and LIVSVM C-SVC tools. MIRG uses the convention of splitting the original dataset into $2 / 3$ for training and testing, with the remaining $1 / 3$ of the data from the original database used for verification (Witten \& Frank, 2005). Because CNN variables have different orders of magnitude, scaling is performed to equalize the importance of all input variables. Variables that are binary, such as $S E X$, have a value of either 1 or -1 . For all other variables, MIRG uses the z-score normalization (Olden \& Jackson, 2002) :

$$
z_{n}=\frac{x_{n}-x}{3 \sigma}
$$

where,

$z_{n}=$ normalized value of variable $x_{n}$

$x_{n}=$ original variable $x$

$\boldsymbol{x}=$ mean of the variable $x$

$\sigma=$ standard deviation of variable $x$

\section{NEC model for the total population}

For the ANN RFW tool datasets, the 13133 case database is divided into three datasets: training, testing and verification. As suggested by Ennett (2001), to deal with the data imbalance due to the low number of NEC cases, the training set is resampled using random oversampling to increase the number of NEC babies to $20 \%$. Resampling involves:

1. From the 13133 database, randomly selecting the NEC cases used in training

2. Making multiple copies of the NEC case selected for the training dataset 
3. Ensuring that all NEC cases (i.e. only NEC cases in the training set) are represented at least once in the training database.

This resampling is done for all models (VLBW and GestAge $<33$ weeks). Table 3.2 summarizes the cases distribution for the three total population datasets.

Table 3.2: ANN RFW data distribution - NEC model for the total population

\begin{tabular}{lccccc}
\hline & \multicolumn{2}{c}{ NEC cases } & \multicolumn{2}{c}{ Normal cases } & TOTAL \\
\cline { 2 - 6 } & $\#$ & $\%$ & $\#$ & $\%$ & $\#$ \\
\hline Original database & 312 & 2.38 & 12821 & 97.62 & 13133 \\
\hline DATASETS & & & & & \\
\hline Training & 1428 & 20.0 & 5712 & 80.0 & 7140 \\
Testing & 69 & 2.36 & 2849 & 97.64 & 2918 \\
Verification & 100 & 2.30 & 4260 & 97.70 & 4360 \\
\hline
\end{tabular}

Due to the $n$-fold cross-validation training of the LIBSVM C-SVM classifier, two training/testing dataset have been created for C-SVC training. The network is first trained and verified with the distribution of NEC and normal cases as found in the original 13133 database. Training and verification is then performed using a resampled training/testing dataset as summarized in table 3.3.

Table 3.3: LIBSVM C-SVC data distribution - NEC model for the total population

\begin{tabular}{lccccc}
\hline & \multicolumn{2}{c}{ NEC cases } & \multicolumn{2}{c}{ Normal cases } & TOTAL \\
\cline { 2 - 6 } & $\#$ & $\%$ & $\#$ & $\%$ & $\#$ \\
\hline Original database & 312 & 2.38 & 12821 & 97.62 & 13133 \\
\hline DATASETS & & & & & \\
\hline Training/Testing & 212 & 2.42 & 8561 & 97.58 & 8773 \\
Training/Testing (resampled) & 2140 & 20.00 & 8561 & 80.00 & 10701 \\
Verification & 100 & 2.30 & 4260 & 97.70 & 4360 \\
\hline
\end{tabular}


It should be pointed out that the exact same cases are used in the training sets for both the ANN RFW and the LIBSVM C-SVM classifier. In addition, the verification sets for both classifiers are made up of identical cases and data distributions. This holds true for the datasets created for subsequent NEC models as well (VLBW and gestation $<33$ weeks). Lastly, the training, testing and verification datasets are independent of one another, i.e. the datasets do not have the same patient records. The datasets were created only once for each tool (ANN and SVM). The work undertaken in thesis does not investigate data resampling on a different training NEC case subset. This can be considered part of future work in terms of database preparation techniques.

\section{NEC in $V L B W$ population}

On the stipulation of literature that different risk factors are associated with NEC in preterm neonates versus those considered term, a NEC model for VLBW babies was considered a valid path to explore. The database used for this model involves a subset of the 13133 case CNN database, where the selection criterion is based on the birth weight being limited to babies $\leq 1,500 \mathrm{~g}$. Tables 3.4 and 3.5 summarize the data split for ANN RFW and LIBSVM C-SVC classifiers respectively.

Table 3.4: ANN RFW data distribution - NEC model for the VLBW population

\begin{tabular}{lccccc}
\hline & \multicolumn{2}{c}{ NEC cases } & \multicolumn{2}{c}{ Normal cases } & TOTAL \\
\cline { 2 - 6 } & $\#$ & $\%$ & $\#$ & $\%$ & $\#$ \\
\hline Original database & 233 & 6.85 & 3170 & 93.15 & 3403 \\
\hline DATASETS & & & & & \\
\hline Training & 353 & 20.0 & 1414 & 80.0 & 1767 \\
Testing & 52 & 6.86 & 706 & 93.14 & 758 \\
Verification & 80 & 7.08 & 1050 & 92.92 & 1130 \\
\hline
\end{tabular}


Table 3.5: LIBSVM C-SVC data distribution - NEC model for the VLBW population

\begin{tabular}{lccccc}
\hline & \multicolumn{3}{c}{ NEC cases } & \multicolumn{3}{c}{ Normal cases } & TOTAL \\
\cline { 2 - 6 } & $\#$ & $\%$ & $\#$ & $\%$ & $\#$ \\
\hline Original database & 233 & 6.85 & 3170 & 93.15 & 3403 \\
\hline DATA SETS & & & & & \\
\hline Training/Testing & 153 & 6.73 & 2120 & 93.27 & 2273 \\
Training/Testing (resampled) & 530 & 20.00 & 2120 & 80.00 & 2650 \\
Verification & 80 & 7.08 & 1050 & 92.92 & 1130 \\
\hline
\end{tabular}

\section{$N E C$ in population with gestation $<33$ weeks}

In addition to the VLBW population that comprises primarily of preterm neonates, the last population of interest considered for the preterm group are the neonates born at less than 33 weeks of gestation. This dataset is also a subset of the 13133 cases CNN database, and just like the VLBW subset database, it has a considerably smaller population of the normal cases, as seen in tables 3.6 and 3.7.

Table 3.6: ANN RFW data distribution - NEC model for gestation age $<33$ weeks

\begin{tabular}{|c|c|c|c|c|c|}
\hline & \multicolumn{2}{|c|}{ NEC cases } & \multicolumn{2}{|c|}{ Normal cases } & \multirow{2}{*}{$\frac{\text { TOTAL }}{\#}$} \\
\hline & \# & $\%$ & $\#$ & $\%$ & \\
\hline Original database & 254 & 5.42 & 4436 & 94.58 & 4690 \\
\hline \multicolumn{6}{|l|}{ DATA SETS } \\
\hline Training & 494 & 20.0 & 1977 & 80.0 & 2471 \\
\hline Testing & 54 & 5.18 & 989 & 94.82 & 1043 \\
\hline Verification & 90 & 5.76 & 1470 & 94.23 & 1560 \\
\hline
\end{tabular}

Table 3.7: LIBSVM C-SVC data distribution - NEC model for gestation age $<33$ weeks

\begin{tabular}{lccccc}
\hline & \multicolumn{3}{c}{ NEC cases } & \multicolumn{3}{c}{ Normal cases } & TOTAL \\
\cline { 2 - 6 } & $\#$ & $\%$ & $\#$ & $\%$ & $\#$ \\
\hline Original database & 254 & 5.42 & 4436 & 94.58 & 4690 \\
\hline DATASETS & & & & & \\
\hline Training/Testing & 164 & 5.24 & 2966 & 94.76 & 3130 \\
Training/Testing (resampled) & 741 & 20.00 & 2966 & 80.00 & 3707 \\
Verification & 90 & 5.76 & 1470 & 94.23 & 1560 \\
\hline
\end{tabular}




\subsection{Finding the Minimum Data Set}

Misuse of current medical scoring systems, for outcomes for which they were not developed, drives the need to develop a minimum data set used in outcome prediction for NEC. Development of the NEC model follows two methodologies: ANN RFW and LIBSVM C-SVC.

\subsubsection{ANN RFW}

Automation of the ANN RFW by Rybchynski (2005) gives the tool the capability to perform an exhaustive search for network parameters and structures. This is done for both the single layer ( 0 hidden layers) and double layer (1 hidden layer) structure. As such, linear and non-linear $(2 n+1, n>0)$ structures are used and analyzed in the development of all the three NEC models. Network training starts with all 27 input variables, and uses weight decay and weight elimination (section 2.3.2.2) to determine the weights of least significance. Although the process is automated, researcher interaction is necessary to ensure that the best structure is selected by the network and that only structures where learning has occurred are selected as those of 'best' performance. Next, the modified Garson-Goh weight reduction method, as developed by Rybchynski (2005), uses the network weights and biases to determine the importance of network input variables. Variables are assigned weights from 0 (lowest) to 100 (highest) and are ranked in descending order of importance. The variables with the lowest weight are considered least significant and are removed from the network. The entire process is repeated, and variables are reduced until network performance degrades. The ANN RFW methodology steps are summarized: 
1. For $N$-input variables, train and validate the network for all $2 n+1$ structures, where $\mathrm{n}=\#$ of input variables and $0<\mathrm{n} \leq N$.

2. Examine the $\log$ sensitivity, sensitivity and specificity graphs to ensure network convergence. Discard structures where oscillation occurs in the graphs. Discard structures were there is a large difference between the training and testing sets. These structures over generalize and validate poorly with respect to the verification dataset.

3. Given that the test epoch is not less than 100 (set by MIRG to ensure sufficient network training (Khan, 2006), ensure that the 'best test epoch' is taken from a steady state on the log sensitivity graph.

4. Upon selecting the 'best structure' obtain the network performance by averaging the results of the 10 verification sets:
i. Sensitivity
ii. Specificity
iii. Classification rate

NOTE: Presence of zero value entries in the validation results suggest that the network has not converged. This means that network validation was not performed. If this is the case, a search for the 'best structure' has to be repeated for the $N$-input variables and another structure selected.

5. Upon successful completion of steps 1 to 4 , invoke Rybchynski's weight reduction method on the weights and biases of the best performing structure. 
6. Rank the $N$-input variables in descending order of importance, as determined by the weights from step 5 .

7. Remove the variable(s) $i_{k}$ (where $k=1, \ldots, N$ ) with the smallest weight, as determined in step 6.

8. Repeat the entire procedure for $\left(N-i_{k}\right)$-input variable set. The minimum data set is obtained when performance from one structure to the next degrades.

\subsubsection{LIBSVM C-SVC}

The radial basis function kernel used in this research, where $K\left(x, x_{i}\right)=\exp (-\gamma \| x-$

$\left.\mathrm{x}_{\mathrm{i}} \|^{2}\right), \gamma>0$, requires optimization of the $C$ and $\gamma$ parameters prior to commencing classification with the LIBSVM C-SVC tool, as these are not known beforehand. The $C$ parameter is weighed with respect to the distribution of the rare and normal outcomes; whereas the gamma parameter $(\gamma)$ is proportional to the width of the Gaussian basis function. It is of highest interest to determine the best $(\mathrm{C}, \gamma)$ parameters so that the classifier can be used for prediction. Chang and Lin (2001) suggest the use of $n$-fold cross-validation and grid search to identify suitable $C$ and $\gamma$, which give a classification rate with a balance between sensitivity and specificity classifications. The authors reported that exponentially increasing sequences of $C$ and $\gamma$ is a practical way of identifying 'good' parameters; hence the grid search is done using $\log _{2} C$ and $\log _{2} \gamma$. Once valid $(C, \gamma)$ parameters are found, the network is trained and tested on them for the different datasets.

A grid search for parameters is a time-consuming and computation-heavy task. By approximation alone, it is impossible to determine the correct starting point for the 
parameter search. Using exponentially growing sequences for $\mathrm{C}$ and $\gamma$ saves time, as does using a 'coarse' grid search with a step size of 1 . Chang and Lin recommend this approach (Chang \& Lin, 2001). For large datasets, such as the one used in this study, the authors also suggested taking a representative set of the population and determining the $(C, \gamma)$ parameter pair for this subset of the original data first. With this in mind, VLBW and GestAge less than 33 weeks data sets are trained and tested first. A 'fine' grid search for the ideal $(\mathrm{C}, \gamma)$ parameter pair is performed once the region of interest is identified successfully with a coarse grid search. The fine grid search is performed with step sizes 0.5 and 0.1 respectively, in order to obtain better classification results.

\subsubsection{Sensitivity analysis (SVM)}

Finding the minimum data with LIBSVM C-SVC tool is a brute force procedure compared to the relative weight reduction method used by the ANN RFW. The standard SVM, as is the case for LIBSVM C-SVC, "can not select variables automatically and therefore its solution typically utilizes all the input variables without discrimination" (Zhang, Liu, Wu, \& Zhu, 2008). Because input variable weights are not calculated, it is difficult to identify important predictor variables. To deal with this issue, this research uses sensitivity analysis in to reduce the initial variable set and obtain the minimum data set. Backward elimination is executed for all input variables, by removing one input variable at a time and training the algorithm. Once the algorithm is trained without a particular input variable, the removed variable is then reinstated to the set, the next variable is removed and the algorithm retrained. The model with the least amount of degradation with respect to specificity, sensitivity and classification rate is selected before the procedure of retraining the SVM is repeated with a smaller variable set. 
Assuming that $C$ and $\gamma$ have been optimized, the steps performed to obtain the minimum data set with SVM are the following:

1. Train the C-SVC network with the $N$-input variable training dataset $(N=27$ for all NEC datasets, except the SNAPPE-II datasets where $N=9$ ).

2. Perform validation with the $N$-input variable verification dataset.

3. Obtain network performance for the verification dataset:

i. Sensitivity

ii. Specificity

iii. Classification rate

4. Remove input variable $i_{k}$ (where $k=1, \ldots, N$ ) from the $N$-input variable training and verification datasets.

5. Repeat steps 1 to 3 : training and validating the network on $N-i_{k}$ variables.

6. Replace variable $i_{k}$ removed in step 4 .

7. Repeat steps 4 to 6 for all $N$-input variables: $i_{k+1}, \ldots, i_{N}$

8. Once training and validation is completed for all $N$-input variables, remove variable $i_{k}$ which gives the best network performance with respect to specificity, sensitivity and classification rate.

9. Repeat the entire procedure for $\left(N-i_{k}\right)$-input variable set. The minimum data set is obtained when performance from one structure to the next degrades. 


\subsubsection{Model Comparison}

With respect to result analysis, model comparison is first performed within each of the two tools. ANN RFW models obtained for the total population (13133 cases), VLBW and less than 33 weeks gestation are compared to each other. The same is done for the models obtained with the LIBSVM C-SVC tool. Comparisons are done with respect to:

1. Performance of the tools for each population

a. network structure

b. sensitivity, specificity and classification rate; where specificity $\geq 85 \%$ is chosen as the selection criteria in order to minimize the number of misclassifications

2. Variables in the minimum data set
a. common variables
b. variables of highest importance
c. SNAPPE-II variables

Once this is complete, performance of the tools is done with respect to each other. Comparison between the two tools is done based on the results from the verification sets, which were kept identical for the ANN RFW and the LIBSVM C-SVC tools. 


\section{RESULTS}

\subsection{Statistical analysis}

Spearman analysis was performed in order to get a better understanding of how the input variables are related to the outcome of NEC. The level of correlation significance was taken as $p<0.05$. From table 4.1 it can be seen that 25 out of 27 input variables had a fairly negligible $\rho$ correlation coefficient, with the exception of birth weight $(\rho=-1.63)$ and gestational age $(\rho=-158)$. This is not a surprise as both low birth weight and low gestational age are associated with a higher risk of NEC. Variables lurine, lgluc, lsodium, hpco 2 and $S E X$ all had large $p$ values $(.680, .144, .225, .536$ and .274 respectively), leading to the conclusion that these variables are independent and not correlated with NEC.

Table 4.1: Spearman correlation and 2-tailed statistical significance of input variables with respect to the outcome of NEC

\begin{tabular}{|l|c|c|c|}
\hline \multicolumn{2}{|c|}{} & \multicolumn{2}{c|}{ NEC } \\
\hline Variable & $\begin{array}{c}\text { SNAPPE-II } \\
\text { variable }(\sqrt{ })\end{array}$ & $\begin{array}{c}\text { Correlation } \\
\text { Coefficient } \\
\rho \text { (rho) }\end{array}$ & $\begin{array}{c}\text { Sig. (2- } \\
\text { tailed) }\end{array}$ \\
\hline lurine & $\sqrt{ }$ & -.004 & .680 \\
\hline hrespr & & $-.097^{* *}$ & .000 \\
\hline lplt & & $-.027^{* *}$ & .002 \\
\hline sga & $\sqrt{ }$ & $.038^{* *}$ & .000 \\
\hline hgluc & & $.044^{* *}$ & .000 \\
\hline lhema & & $-.081^{* *}$ & .000 \\
\hline Apgar5 & $\sqrt{ }$ & $-.060^{* *}$ & .000 \\
\hline lbloodp & $\sqrt{ }$ & $-.097^{* *}$ & .000 \\
\hline hhema & & $-.075^{* *}$ & .000 \\
\hline lgluc & & .013 & .144 \\
\hline birthweight & $\sqrt{ }$ & $-.163^{* *}$ & .000 \\
\hline hsodium & & $-.020^{*}$ & .023 \\
\hline hitnrat & & $.058^{* *}$ & .000 \\
\hline
\end{tabular}




\begin{tabular}{|l|c|c|c|}
\hline LWBC & & $-.082^{* *}$ & .000 \\
\hline LANC & & $-.083^{* *}$ & .000 \\
\hline lheartr & & $.076^{* *}$ & .000 \\
\hline hheartr & & $.073^{* *}$ & .000 \\
\hline OI & & $.049^{* *}$ & .000 \\
\hline apnea & & $.025^{* *}$ & .005 \\
\hline ltempf & $\sqrt{ }$ & $-.034^{* *}$ & .000 \\
\hline seizure & $\sqrt{ }$ & $-.026^{* *}$ & .003 \\
\hline lsodium & & -.011 & .225 \\
\hline lpo2po2 & & $.020^{*}$ & .019 \\
\hline lserum & $\sqrt{ }$ & $-.036^{* *}$ & .000 \\
\hline Po2fio2r & $\sqrt{ }$ & $-.048^{* *}$ & .000 \\
\hline hpco2 & & .005 & .536 \\
\hline GestAGE & & $-.158^{* *}$ & .000 \\
\hline SEX & & -.010 & .274 \\
\hline
\end{tabular}

**. Correlation is significant at the 0.01 level (2-tailed).

*. Correlation is significant at the 0.05 level (2-tailed).

\subsection{ANN RFW Models}

\subsubsection{Total population model}

Two NEC models were analyzed for the 13133 case database. In the first model, model A, the GestAge variable was classified with respect to 33 weeks of gestation. A value of -1 was assigned to babies born at or above 33 weeks of gestation; a value of 1 was assigned to babies born at less than 33 weeks of gestation. The purpose of differentiating by gestational age using categories, as opposed to scaling the variable with the z-score normalization, was to enhance the performance of the network. This is due to the fact that most babies with NEC were found to be born at less than 33 weeks of gestation (254 out of 312). The gestational split is considered significant primarily because NEC is thought to be more abundant in preterm babies. ANN results and variables sets for this model are shown in table 4.2 and 4.3 respectively. 
Table 4.2: ANN RFW results for Model A*

\begin{tabular}{|l|l|l|l|l|l|l|l|}
\hline \# of Variables & $\mathbf{2 7}$ & 23 & $\mathbf{2 0}$ & $\mathbf{1 9}$ & $\mathbf{1 8}$ & 17 \\
\hline Best test epoch & & 711 & 672 & 1201 & $\mathbf{9 2 1}$ & 1069 & 1080 \\
\hline Best structure & 43 & 25 & 7 & $\mathbf{6}$ & 10 & 30 \\
\hline \multirow{2}{*}{ Log sensitivity } & Best test & 0.2666 & 0.4169 & 0.3327 & $\mathbf{0 . 4 0 8 1}$ & 0.4110 & 0.3879 \\
\cline { 2 - 8 } & Verification avg. & $\mathbf{0 . 2 2 2 9}$ & 0.3196 & 0.2518 & $\mathbf{0 . 3 5 6 1}$ & 0.4410 & 0.2649 \\
\hline \multirow{2}{*}{ Sensitivity (\%) } & Best test & 71.01 & $\mathbf{8 2 . 6 1}$ & 76.81 & $\mathbf{8 2 . 6 1}$ & $\mathbf{8 2 . 6 2}$ & $\mathbf{8 2 . 6 1}$ \\
\cline { 2 - 8 } & Verification avg. & $\mathbf{6 8 . 0 0}$ & 74.00 & 71.00 & $\mathbf{7 6 . 0 0}$ & $\mathbf{7 4 . 0 0}$ & 73.00 \\
\hline \multirow{2}{*}{ Specificity (\%) } & Best test & $\mathbf{8 1 . 5 0}$ & $\mathbf{8 3 . 1 9}$ & $\mathbf{8 2 . 1 7}$ & $\mathbf{8 2 . 2 4}$ & $\mathbf{8 2 . 5 6}$ & $\mathbf{8 2 . 2 4}$ \\
\cline { 2 - 8 } & Verification avg. & 79.80 & $\mathbf{8 3 . 9 2}$ & 79.81 & $\mathbf{8 2 . 1 6}$ & $\mathbf{8 0 . 6 3}$ & 81.76 \\
\hline \multirow{2}{*}{ CCR (\%) } & Best test & 81.25 & 83.17 & 82.04 & $\mathbf{8 2 . 2 5}$ & 82.56 & $\mathbf{8 2 . 2 5}$ \\
\cline { 2 - 8 } & Verification avg. & 79.54 & 83.69 & 79.61 & $\mathbf{8 2 . 0 2}$ & 80.48 & 81.56 \\
\hline \multirow{2}{*}{ ROC } & Best test & $\mathbf{8 0 . 4 9}$ & 84.99 & 84.89 & $\mathbf{8 6 . 9 5}$ & 86.11 & 84.78 \\
\cline { 2 - 8 } & Verification avg. & 71.88 & 69.71 & 72.53 & $\mathbf{7 5 . 6 9}$ & 76.08 & 72.40 \\
\hline
\end{tabular}

*Model A - GestAge category of $[-1,1]$

Table 4.3: Variables and weights for best performance structures for Model $A^{*}$

\begin{tabular}{|c|c|c|c|c|c|c|c|c|c|c|c|}
\hline \multicolumn{2}{|c|}{27 variables } & \multicolumn{2}{|c|}{23 variables } & \multicolumn{2}{|c|}{20 variables } & \multicolumn{2}{|c|}{19 variables } & \multicolumn{2}{|c|}{18 variables } & \multicolumn{2}{|c|}{17 variables } \\
\hline Igluc & 100 & Igluc & 100 & GEST & 100 & bthwt & 100 & bthwt & 100 & bthwt & 100 \\
\hline seizure & 96 & hrespr & 93 & Apgar 5 & 72 & seizure & 67 & hrespr & 86 & LWBC & 99 \\
\hline Lurine & 94 & OI & 90 & Po2fio2r & 67 & hrespr & 63 & hpco2 & 58 & hrespr & 90 \\
\hline Apgar5 & 94 & Ipo2po2 & 89 & hrespr & 63 & Apgar5 & 61 & Po2fio2r & 53 & GEST & 86 \\
\hline hitnrat & 93 & hpco2 & 86 & hpco2 & 62 & lserum & 51 & hgluc & 49 & apnea & 86 \\
\hline hrespr & 93 & LWBC & 86 & bthwt & 57 & hitnrat & 50 & Apgar5 & 44 & seizure & 86 \\
\hline hsodium & 92 & Lurine & 84 & lplt & 57 & hsodium & 44 & ltempf & 41 & OI & 84 \\
\hline Po2fio2r & 90 & hgluc & 82 & hsodium & 52 & apnea & 40 & hitnrat & 40 & hitnrat & 84 \\
\hline apnea & 89 & hsodium & 80 & apnea & 47 & OI & 39 & lserum & 39 & hsodium & 84 \\
\hline hheartr & 89 & hitnrat & 77 & seizure & 44 & ltempf & 38 & Lurine & 37 & hgluc & 83 \\
\hline GEST & 86 & seizure & 76 & ltempf & 43 & lplt & 34 & LWBC & 37 & hpco2 & 79 \\
\hline lheartr & 85 & Po2fio2r & 76 & Igluc & 42 & lgluc & 33 & apnea & 37 & Po2fio2r & 79 \\
\hline hpco2 & 85 & bthwt & 76 & hgluc & 37 & Lurine & 29 & GEST & 34 & lserum & 78 \\
\hline ltempf & 85 & GEST & 73 & lserum & 36 & hpco2 & 24 & Igluc & 34 & Lurine & 75 \\
\hline LANC & 85 & lserum & 73 & 1po2po2 & 34 & Po2fio2r & 21 & OI & 34 & ltempf & 73 \\
\hline hgluc & 84 & apnea & 71 & OI & 33 & GEST & 20 & seizure & 30 & Apgar5 & 65 \\
\hline OI & 83 & Apgar5 & 66 & Lurine & 33 & LWBC & 17 & hsodium & 27 & Igluc & 59 \\
\hline hhema & 83 & ltempf & 66 & hitnrat & 32 & hgluc & 16 & lplt & 17 & & \\
\hline lserum & 82 & lplt & 65 & LWBC & 31 & lpo2po2 & 14 & & & & \\
\hline LWBC & 81 & hhema & 65 & hhema & 27 & & & & & & \\
\hline bthwt & 80 & hheartr & 59 & & & & & & & & \\
\hline lpo2po2 & 80 & $L A N C$ & 57 & & & & & & & & \\
\hline lplt & 80 & lheartr & 57 & & & & & & & & \\
\hline lhema & 79 & & & & & & & & & & \\
\hline SEX & 78 & & & & & & & & & & \\
\hline lbloodp & 75 & & & & & & & & & & \\
\hline Isodium & 67 & & & & & & & & & & \\
\hline
\end{tabular}

*Model A - GestAge category of $[-1,1]$ 
From table 4.2 it can be seen that the reduction of variables results in a fairly steady network performance with respect to the sensitivity, specificity and classification rates. The testing specificity and sensitivity values are nearly matching each other within each structure. However, this is not the case for the verification results. The difference between the testing and verification sensitivities is the smallest in the 27 variable dataset, coming in at $71.01 \%$ to $68.00 \%$ respectively. The conclusion here is that the network overtrains and does not generalize well to the rest of the population. The most significant variables for the model were hrespr, lgluc, and birthweight. Hrespr stayed fairly significant for the entire variable reduction, whereas lgluc level of significance decreased over the duration of the experiments. Birthweight picked up significance as the variable dataset decreased in size; in fact birthweight remained as the top variable in the 19, 18 and 17 variable models.

The second model, model B, had the gestation variable scaled using the $\mathrm{z}$-score normalization equation (section 3.1.1, equation 3.1) instead of using the category split of Model A. This was done to improve on the verification results, which Model B lacked in. The results can be seen in table 4.4 and 4.5 .

Table 4.4: ANN RFW results for Model B*

\begin{tabular}{|c|c|c|c|c|c|c|c|c|}
\hline \multicolumn{2}{|c|}{ \# of Variables } & 27 & 25 & 24 & 22 & 21 & 20 & 19 \\
\hline \multicolumn{2}{|c|}{ Best test epoch } & 1494 & 239 & 540 & 1055 & 708 & 1014 & 783 \\
\hline \multicolumn{2}{|c|}{ Best structure } & 11 & 12 & 43 & 13 & 9 & 13 & 10 \\
\hline \multirow{2}{*}{$\begin{array}{l}\text { Log } \\
\text { sensitivity }\end{array}$} & Best test & 0.2706 & 0.1856 & 0.3024 & 0.2387 & 0.3018 & 0.3271 & 0.2994 \\
\hline & Verification avg. & 0.1864 & 0.1805 & 0.1706 & 0.1461 & 0.1814 & 0.2390 & 0.1917 \\
\hline \multirow{2}{*}{$\begin{array}{l}\text { Sensitivity } \\
(\%)\end{array}$} & Best test & 68.12 & 59.42 & 71.02 & 65.22 & 71.01 & 73.91 & 71.01 \\
\hline & Veri & \begin{tabular}{|l|}
64.00 \\
\end{tabular} & 61.00 & 67.00 & 63.00 & 65.00 & 67.00 & 64.00 \\
\hline \multirow{2}{*}{$\begin{array}{l}\text { Specificity } \\
\text { (\%) }\end{array}$} & Best & 88.00 & 86.31 & 88.00 & 87.33 & 87.89 & 86.45 & 87.47 \\
\hline & Verif & 87.75 & 78.85 & 81.20 & 84.79 & 85.94 & 86.74 & 86.95 \\
\hline \multirow[t]{2}{*}{ CCR (\%) } & Best $t$ & 87.56 & 85.68 & 87.59 & 86.81 & 87.49 & 86.15 & 87.08 \\
\hline & Verif & 87.20 & 78.44 & 80.87 & 84.29 & 85.46 & 86.28 & 86.42 \\
\hline \multirow[t]{2}{*}{ ROC } & Best test & 83.06 & 84.79 & 84.31 & 83.68 & 83.63 & 83.73 & 84.52 \\
\hline & Verification & 72.51 & 75.38 & 76.61 & 74.30 & 74.24 & 74.61 & 74.64 \\
\hline
\end{tabular}

*Model B - GestAge scaled by z-score normalization 
Table 4.5: Variables and weights for best performance structures for Model $\mathrm{B}^{*}$

\begin{tabular}{|c|c|c|c|c|c|c|c|c|c|c|c|c|c|}
\hline \multicolumn{2}{|c|}{27 variables } & \multicolumn{2}{|c|}{25 variables } & \multicolumn{2}{|c|}{24 variables } & \multicolumn{2}{|c|}{22 variables } & \multicolumn{2}{|c|}{21 variables } & \multicolumn{2}{|c|}{20 variables } & \multicolumn{2}{|c|}{19 variables } \\
\hline GEST & 100 & lgluc & 100 & lbloodp & 100 & Igluc & 100 & bthwt & 100 & GEST & 100 & bthwt & 100 \\
\hline OI & 87 & lserum & 88 & hitnrat & 92 & Po2fio2r & 99 & hhema & 91 & bthwt & 85 & Po2fio2r & 99 \\
\hline hhema & 84 & hitnrat & 79 & lheartr & 88 & $\mathrm{OI}$ & 99 & LANC & 90 & seizure & 77 & GEST & 98 \\
\hline lsodium & 84 & lsodium & 77 & Apgar5 & 87 & apnea & 95 & lbloodp & 86 & lsodium & 76 & hitnrat & 83 \\
\hline Igluc & 84 & OI & 67 & LANC & 86 & GEST & 88 & hheartr & 84 & hpco2 & 70 & hhema & 80 \\
\hline Iserum & 83 & lbloodp & 64 & Isodium & 84 & lheartr & 82 & lsodium & 77 & OI & 68 & LANC & 73 \\
\hline Lurine & 83 & GEST & 63 & Lurine & 83 & Apgar5 & 82 & GEST & 75 & LANC & 66 & lbloodp & 71 \\
\hline SEX & 82 & lhema & 63 & Igluc & 81 & hheartr & 79 & Apgar5 & 74 & apnea & 62 & OI & 66 \\
\hline hheartr & 80 & lheartr & 63 & OI & 81 & hitnrat & 78 & lhema & 72 & Po2fio2r & 57 & apnea & 64 \\
\hline hpco 2 & 78 & ltempf & 62 & bthwt & 81 & lsodium & 72 & Iserum & 71 & lserum & 54 & lhema & 63 \\
\hline apnea & 78 & Lurine & 60 & Iserum & 80 & 1tempf & 71 & OI & 71 & Igluc & 54 & hpco2 & 63 \\
\hline lplt & 76 & LANC & 60 & 1po2po2 & 78 & LANC & 70 & apnea & 71 & Lurine & 53 & lserum & 62 \\
\hline seizure & 76 & hheartr & 59 & GEST & 77 & bthwt & 68 & hpco2 & 70 & ltempf & 51 & Apgar5 & 57 \\
\hline LANC & 75 & hhema & 56 & apnea & 77 & hhema & 68 & hitnrat & 66 & lhema & 50 & Lurine & 56 \\
\hline lbloodp & 74 & hpco2 & 56 & hheartr & 76 & lbloodp & 68 & seizure & 66 & Apgar5 & 49 & lgluc & 52 \\
\hline hsodium & 73 & lplt & 55 & Po2fio2r & 75 & hpco2 & 66 & Igluc & 62 & lbloodp & 48 & lsodium & 45 \\
\hline 1po2po2 & 71 & apnea & 54 & Itempf & 72 & lserum & 63 & Lurine & 59 & hhema & 46 & seizure & 45 \\
\hline lhema & 68 & 1po2po2 & 53 & lhema & 71 & seizure & 61 & Itempf & 58 & lheartr & 43 & ltempf & 38 \\
\hline Po2fio2r & 67 & seizure & 52 & hhema & 70 & lplt & 61 & lheartr & 57 & hitnrat & 36 & lheartr & 37 \\
\hline ltempf & 66 & Po $2 f i o 2 r$ & 50 & hpco 2 & 70 & lhema & 57 & Po2fio2r & 46 & hheartr & 26 & & \\
\hline bthwt & 66 & bthwt & 47 & seizure & 65 & Lurine & 39 & lplt & 37 & & & & \\
\hline lheartr & 63 & hgluc & 46 & lplt & 65 & lpo2po2 & 38 & & & & & & \\
\hline Apgar5 & 61 & Apgar5 & 41 & hsodium & 64 & & & & & & & & \\
\hline hitnrat & 61 & hsodium & 40 & hgluc & 61 & & & & & & & & \\
\hline hgluc & 61 & $S E X$ & 37 & & & & & & & & & & \\
\hline hrespr & 60 & & & & & & & & & & & & \\
\hline$\angle W B C$ & 57 & & & & & & & & & & & & \\
\hline
\end{tabular}

*Model B - GestAge scaled by z-score normalization

The performance of Model B with respect to verification sensitivity across the minimum data sets (range from $61 \%$ to $67 \%$ ) was worse than that of Model A (range from $68 \%$ to $76 \%$ ). However, the verification results were much better when compared to the testing sensitivity, which means that the Model B network did not overtrain like the Model A network. Both lgluc and birthweight variables were of high importance, just like in the previous model. GestAge also seemed to carry more importance for Model B than Model A. 


\subsubsection{VLBW model}

The VLBW model had the least number of cases of the three models. However, it still met the requirement of having at least 10 times the number of cases as it does variables, so it was considered valid (Masters, 1993). The performance measures and variable reduction are shown in tables 4.6 and 4.7 respectively.

Table 4.6: ANN RFW results for the VLBW model

\begin{tabular}{|l|l|l|l|l|l|l|}
\hline \# of Variables & $\mathbf{2 7}$ & $\mathbf{2 4}$ & $\mathbf{2 3}$ & $\mathbf{2 2}$ & 21 \\
\hline Best test epoch & & 350 & 243 & $\mathbf{4 7 8}$ & 289 & 1784 \\
\hline Best structure & 18 & 24 & $\mathbf{1 3}$ & 18 & 22 \\
\hline \multirow{2}{*}{ Log sensitivity } & Best test & 0.0458 & 0.0566 & $\mathbf{0 . 0 5 8 9}$ & $\mathbf{0 . 0 2 5 4}$ & $\mathbf{0 . 0 4 5 9}$ \\
\cline { 2 - 7 } & Verification avg. & $\mathbf{0 . 0 8 4 8}$ & 0.0674 & $\mathbf{0 . 0 2 9 8}$ & 0.0825 & 0.0226 \\
\hline \multirow{3}{*}{ Sensitivity (\%) } & Best test & 30.77 & 34.62 & $\mathbf{3 4 . 6 2}$ & 23.077 & 30.77 \\
\cline { 2 - 7 } & Verification avg. & $\mathbf{2 8 . 7 5}$ & $\mathbf{3 1 . 2 5}$ & $\mathbf{3 2 . 5 0}$ & $\mathbf{2 7 . 5 0}$ & $\mathbf{2 8 . 7 5}$ \\
\hline \multirow{2}{*}{ Specificity (\%) } & Best test & $\mathbf{9 4 . 4 8}$ & 90.65 & $\mathbf{9 3 . 6 3}$ & 97.02 & 94.48 \\
\cline { 2 - 7 } & Verification avg. & $\mathbf{9 2 . 7 6}$ & $\mathbf{8 8 . 1 0}$ & $\mathbf{8 7 . 0 5}$ & 92.95 & 92.86 \\
\hline \multirow{2}{*}{ CCR (\%) } & Best test & 90.10 & 86.81 & $\mathbf{8 9 . 5 8}$ & 91.95 & 90.11 \\
\cline { 2 - 7 } & Verification avg. & $\mathbf{8 8 . 2 3}$ & 84.07 & $\mathbf{8 3 . 1 9}$ & $\mathbf{8 8 . 3 2}$ & $\mathbf{8 8 . 3 2}$ \\
\hline \multirow{2}{*}{ ROC } & Best test & 54.21 & 58.57 & $\mathbf{6 8 . 0 3}$ & 64.15 & 62.33 \\
\cline { 2 - 7 } & Verification avg. & 56.84 & 54.46 & $\mathbf{6 0 . 6 3}$ & 52.32 & 58.64 \\
\hline
\end{tabular}

Table 4.7: Variables and weights for best performance structures of the VLBW model

\begin{tabular}{|c|c|c|c|c|c|c|c|c|c|}
\hline 27 variables & & 24 variables & & 23 variables & & 22 variables & & 21 variables & \\
\hline bthwt & 100 & hpco2 & 100 & bthwt & 100 & bthwt & 100 & lhema & 100 \\
\hline OI & 88 & lserum & 84 & Apgar5 & 94 & Apgar5 & 90 & hitnrat & 90 \\
\hline Po2fio2r & 84 & Apgar5 & 82 & GestAge & 88 & apnea & 89 & hheartr & 90 \\
\hline lheartr & 81 & bthwt & 79 & SEX & 87 & hitnrat & 86 & SEX & 88 \\
\hline GestAge & 80 & hgluc & 77 & lheartr & 80 & OI & 86 & lserum & 85 \\
\hline LWBC & 80 & LANC & 76 & hgluc & 80 & lsodium & 80 & lplt & 84 \\
\hline Apgar5 & 80 & lsodium & 71 & hheartr & 77 & lhema & 78 & Itempf & 80 \\
\hline lhema & 78 & lhema & 71 & ltempf & 75 & hgluc & 78 & LWBC & 79 \\
\hline SEX & 74 & hhema & 69 & LWBC & 71 & lheartr & 75 & OI & 76 \\
\hline hitnrat & 74 & ltempf & 69 & Lurine & 71 & SEX & 72 & lpo2po2 & 76 \\
\hline lbloodp & 74 & lgluc & 69 & lhema & 67 & LANC & 72 & hpco2 & 74 \\
\hline hgluc & 72 & apnea & 66 & lplt & 66 & lpo2po2 & 68 & lsodium & 71 \\
\hline lplt & 71 & lheartr & 66 & LANC & 65 & lplt & 67 & Lurine & 68 \\
\hline hheartr & 71 & Lurine & 66 & hitnrat & 62 & ltempf & 65 & bthwt & 64 \\
\hline lsodium & 70 & Iplt & 65 & Iserum & 61 & hheartr & 63 & lheartr & 64 \\
\hline hhema & 70 & SEX & 65 & Po2fio2r & 61 & hhema & 63 & LANC & 63 \\
\hline apnea & 68 & GestAge & 64 & hhema & 59 & hpco2 & 62 & hhema & 63 \\
\hline
\end{tabular}




\begin{tabular}{llllllllll}
\hline Lurine & 65 & hitnrat & 63 & lpo2po2 & 59 & lserum & 62 & GestAge & 60 \\
lpo2po2 & 64 & LWBC & 62 & OI & 58 & GestAge & 60 & hgluc & 59 \\
LANC & 62 & Po2fio2r & 62 & hpco2 & 57 & LWBC & 60 & apnea & 58 \\
hpco2 & 60 & lpo2po2 & 61 & apnea & 54 & Lurine & 56 & Apgar5 & 52 \\
lgluc & 58 & hheartr & 61 & lsodium & 49 & Po2fio2r & 52 & & \\
lserum & 57 & OI & 56 & lgluc & 33 & & & & \\
ltempf & 52 & lbloodp & 46 & & & & & & \\
seizure & 47 & & & & & & & & \\
hrespr & 47 & & & & & & & & \\
hsodium & 45 & & & & & &
\end{tabular}

There was a drastic change in the sensitivity level of the VLBW model compared to sensitivities observed in models A and B. In fact, sensitivity of this model was nearly one half of Model A. This could potentially be attributed to the fact that the VLBW population is made up of babies that have additional medical complications. The complications may confound with those of NEC, making it hard for the network to distinguish between the two populations. However, this is a speculation that needs further investigation. The importance of Apgar 5 and $b t h w t$ variables was particularly pronounced in the VLBW model. Both of the variables ranked high for the $27,24,23$ and 22 variable models.

\subsubsection{GestAge $<33$ weeks model}

The last model analyzed was for the CNN population whose gestational age was less than 33 weeks. The results of this model can be seen in table 4.8 and 4.9 respectively. The model encompasses majority of the cases from the VLBW model, and because of that, the minimum data sets are expected to be similar for the two models. Results observed with respect to sensitivity, specificity and classification rates in the VLBW model are expected for this one as well. 
Table 4.8: ANN RFW results for the GestAge $<33$ weeks population

\begin{tabular}{|l|l|l|l|l|l|l|}
\hline \# of Variables & $\mathbf{2 7}$ & $\mathbf{2 2}$ & $\mathbf{2 1}$ & $\mathbf{2 0}$ & $\mathbf{1 9}$ \\
\hline Best test epoch & 538 & 919 & 631 & $\mathbf{6 0 9}$ & 1409 \\
\hline Best structure & Best test & 10 & $\mathbf{8}$ & 8 & $\mathbf{6}$ & 5 \\
\hline \multirow{2}{*}{ Log sensitivity } & Verification avg. & 0.0682 & 0.0511 & 0.0636 & $\mathbf{0 . 0 6 7 0}$ & 0.0976 \\
\hline \multirow{2}{*}{ Sensitivity (\%) } & Best test & 37.0911 & 0.0584 & 0.1179 & $\mathbf{0 . 0 8 4 0}$ & 0.0390 \\
\cline { 2 - 7 } & Verification avg. & $\mathbf{3 3 . 3 3}$ & $\mathbf{3 1 . 1 1}$ & $\mathbf{3 0 . 0 0}$ & $\mathbf{3 3 . 3 3}$ & $\mathbf{3 7 . 7 8}$ \\
\hline \multirow{2}{*}{ Specificity (\%) } & Best test & 93.33 & $\mathbf{8 9 . 2 8}$ & $\mathbf{8 9 . 1 8}$ & $\mathbf{9 2 . 0 1}$ & $\mathbf{8 9 . 2 8}$ \\
\cline { 2 - 7 } & Verification avg. & 89.05 & 88.30 & $\mathbf{8 8 . 5 7}$ & $\mathbf{8 6 . 2 6}$ & 86.46 \\
\hline \multirow{2}{*}{ CCR (\%) } & Best test & 90.41 & 86.38 & 85.52 & $\mathbf{8 9 . 1 7}$ & 86.96 \\
\cline { 2 - 7 } & Verification avg. & 85.83 & 85.00 & 85.19 & $\mathbf{8 3 . 2 1}$ & 83.65 \\
\hline \multirow{2}{*}{ ROC } & Best test & 63.07 & 60.59 & 56.89 & $\mathbf{6 0 . 7 7}$ & 63.73 \\
\cline { 2 - 7 } & Verification avg. & 60.28 & 50.07 & 59.28 & $\mathbf{6 0 . 0 8}$ & 57.57 \\
\hline
\end{tabular}

Table 4.9: Variables and weights for best performance structures of the GestAge $<33$ weeks model

\begin{tabular}{|c|c|c|c|c|c|c|c|c|c|}
\hline 27 variables & & 22 variables & & 21 variables & & 20 variables & & 19 variables & \\
\hline Igluc & 100 & GEST & 100 & hitnrat & 100 & bthwt & 100 & lbloodp & 100 \\
\hline hitnrat & 100 & LWBC & 78 & lserum & 85 & Po2fio2r & 99 & hhema & 89 \\
\hline Lurine & 98 & bthwt & 73 & Apgar5 & 69 & lhema & 90 & bthwt & 82 \\
\hline Apgar5 & 98 & ltempf & 68 & bthwt & 67 & lgluc & 87 & hitnrat & 78 \\
\hline hsodium & 95 & 1serum & 67 & GEST & 66 & hheartr & 84 & Apgar5 & 75 \\
\hline LWBC & 91 & Lurine & 67 & hrespr & 66 & hgluc & 79 & GEST & 68 \\
\hline ltempf & 91 & lhema & 65 & SEX & 63 & Apgar5 & 76 & LWBC & 68 \\
\hline lbloodp & 90 & hrespr & 63 & hhema & 62 & lbloodp & 75 & hgluc & 66 \\
\hline hhema & 89 & hsodium & 61 & LWBC & 61 & Lurine & 70 & lserum & 64 \\
\hline bthwt & 86 & Apgar5 & 60 & lhema & 59 & GEST & 69 & Po2fio2r & 61 \\
\hline hrespr & 82 & hitnrat & 58 & ltempf & 55 & lserum & 65 & lgluc & 61 \\
\hline lheartr & 80 & LANC & 58 & hgluc & 54 & 1po2po2 & 60 & SEX & 60 \\
\hline lplt & 79 & hgluc & 57 & LANC & 53 & hrespr & 53 & hrespr & 60 \\
\hline GEST & 77 & hheartr & 55 & lpo2po2 & 49 & LANC & 52 & hheartr & 58 \\
\hline Po2fio2r & 76 & lgluc & 52 & Po2fio2r & 47 & LWBC & 51 & Lurine & 57 \\
\hline hheartr & 74 & SEX & 51 & Lurine & 45 & hhema & 45 & $1 \mathrm{po} 2 \mathrm{po} 2$ & 54 \\
\hline 1po2po2 & 73 & Po2fio2r & 49 & lgluc & 45 & SEX & 45 & lhema & 43 \\
\hline LANC & 72 & lplt & 48 & hsodium & 44 & hsodium & 43 & hsodium & 34 \\
\hline SEX & 72 & 1po2po2 & 46 & hheartr & 42 & hitnrat & 43 & LANC & 20 \\
\hline 1serum & 71 & lbloodp & 43 & lbloodp & 37 & ltempf & 28 & & \\
\hline hgluc & 71 & hhema & 41 & lplt & 33 & & & & \\
\hline lhema & 70 & lheartr & 34 & & & & & & \\
\hline seizure & 67 & & & & & & & & \\
\hline hpco2 & 67 & & & & & & & & \\
\hline apnea & 64 & & & & & & & & \\
\hline lsodium & 64 & & & & & & & & \\
\hline OI & 60 & & & & & & & & \\
\hline
\end{tabular}


As expected, the performance of the GestAge $<33$ weeks model was fairly similar to the VLBW model. The GestAge $<33$ weeks also placed a high emphasis on the Apgar 5 variable, as well as the bthwt. However, the same variables were not removed from the VLBW and GestAge $<33$ models.

\subsection{LIBSVM C-SVC Models}

The results and analysis of the LIBSVM C-SVC models for NEC are reported in the inverse order, compared to the results obtained with the ANN RFW tool. The reason for this is the order in which the grid search was performed for obtaining the optimal $C$ and $\gamma$ parameters for the three models. $C$, a regularization constant that determines the trade-off between training errors and rigid margins, is vital for allowing misclassifications (i.e. slightly favouring sensitivity to specificity). The gamma parameter $(\gamma)$ is used by the RBF kernel function. The search for these parameters was initially attempted with the complete database. However, this proved to be a complex task as the grid search is what determines the computational time of the SVM experiments. In order to find the starting point for the search, the preliminary grid search needs to cover a large area. This is a very costly search for large datasets. As such, a smaller subset of the original data was taken, such as the VLBW dataset, in order to establish a grid search starting point. There are two benefits to this approach: 1) it makes much less computational time as the dataset is smaller resulting in faster training, and 2) it is a good estimator of the starting point in the grid search. The preliminary grid searches are considered 'coarse' searches, as they cover a large area. 
As per the suggestion of Chang and Lin (2001), a 'fine' grid search was attempted for all models once the optimal $C$ and $\gamma$ were found. The step size used was both 0.5 and 0.1. An improvement to the classification rate from the two 'fine' grid searches was minimal.

\subsubsection{VLBW model}

Table 4.10: Grid search for the $\mathrm{C}$ and $\gamma$ parameters for VLBW

\begin{tabular}{|c|c|c|c|c|c|}
\hline & \multicolumn{4}{|c|}{ Parameters } & \multirow{2}{*}{$\begin{array}{c}\text { Classification } \\
\text { Rate (\%) }\end{array}$} \\
\hline & $\mathrm{C}$ & $\log _{2} C$ & $\gamma$ & $\log _{2} \gamma$ & \\
\hline $\begin{array}{l}\text { Original training } \\
(\mathrm{NEC}=6.73 \%)\end{array}$ & 4096 & 12.0 & 0.0078123 & -7.0 & 93.40 \\
\hline $\begin{array}{l}\text { Resampled training } \\
(\mathrm{NEC}=20 \%)\end{array}$ & 1.0 & 0.0 & 16.0 & 4.0 & 99.06 \\
\hline $\begin{array}{l}\text { SNAPPE training } \\
(\mathrm{NEC}=20 \%)\end{array}$ & 0.5 & -1 & 4.0 & 2.0 & 93.27 \\
\hline
\end{tabular}

The original VLBW NEC population was $6.73 \%$. This distribution was used in the preliminary 'coarse' grid search for the $\mathrm{C}$ and $\gamma$ parameters (table 4.10). With the LIBSVM C-SVC tool, the user has the ability to assign a penalty weight for the normals in the population. This penalty is determined by the ratio of the NEC babies to the normal population. Just like the logsens index in the ANN RFW tool favours sensitivity at the expense of specificity, the penalty weight is meant to do the same. After finding the optimal parameters, and obtaining a classification rate of 93.40\%, the LIBSVM CSVC was invoked to test and verify the parameters. Verification results were less than satisfactory: classification rate for training and testing was observed to be $92.92 \%$. However, this was only representative of the normals in the population (specificity $=100 \%$ ) as none of the NEC babies were classified correctly (sensitivity $=0 \%$ ). 
To overcome this obstacle, the SVM training data set was resampled in order to increase the distribution of the NEC babies. The classification rate for the 27 variables with the resampled training dataset was $99.06 \%$. However, the VLBW failed in validation once again. The removal of variables one by one, did not seem to improve the sensitivity of the network, which was consistently $0-2 \%$. As such, sensitivity analysis to determine a minimum data set was rendered useless for the VLBW model.

\subsubsection{GestAge $<33$ weeks model}

GestAge $<33$ weeks model encountered the same problems observed in the VLBW model. Validation of the results was rendered impossible after finding the optimal $C$ and $\gamma$ parameters (table 4.11 ).

Table 4.11: Grid search for the $C$ and $\gamma$ parameters for GestAge $<33$ weeks

\begin{tabular}{lccccc}
\hline & \multicolumn{4}{c}{ Parameters } & Classification \\
\cline { 2 - 5 } & $\mathrm{C}$ & $\log _{2} \mathbf{C}$ & $\gamma$ & $\log _{2} \gamma$ & Rate (\%) \\
\hline $\begin{array}{l}\text { Original training } \\
\text { (NEC=5.42\%) }\end{array}$ & 4.0 & 2.0 & 0.5 & -1.0 & 94.95 \\
$\begin{array}{l}\text { Resampled training } \\
\text { (NEC=20\%) }\end{array}$ & 1.0 & 0.0 & 16.0 & 4.0 & 99.73 \\
$\begin{array}{l}\text { SNAPPE training } \\
\text { (NEC=20\%) }\end{array}$ & 2.0 & 1.0 & 1.0 & 0.0 & 94.76 \\
\hline
\end{tabular}

\subsubsection{Total population model}

The total population SVM model was of limited success as well. The original dataset with $\mathrm{NEC}=2.42 \%$ was essentially invalid as the verification was giving sensitivity of less than $3 \%$, and specificity of $100 \%$. Once again it was impossible to determine the variables of importance, and the minimum data set search was deemed futile. Results improved drastically once the database was resampled to increase the 
number of NEC cases from $2.42 \%$ to $20 \%$. Table 4.12 shows the optimum values for parameters of $C$ and $\gamma$; table 4.13 shows the performance measures of the models given in table 4.14, as obtained by the LIBSVM C-SVC tool.

Table 4.12: Grid search for the $\mathrm{C}$ and $\gamma$ parameters for the total population

\begin{tabular}{|c|c|c|c|c|c|}
\hline & \multicolumn{4}{|c|}{ Parameters } & \multirow{2}{*}{$\begin{array}{l}\text { Classification } \\
\text { Rate (\%) }\end{array}$} \\
\hline & C & $\log _{2} C$ & $\gamma$ & $\log _{2} \gamma$ & \\
\hline $\begin{array}{l}\text { Original training } \\
(\mathrm{NEC}=2.42 \%)\end{array}$ & 2.5 & 1.32 & 2.0 & 1.0 & 97.62 \\
\hline $\begin{array}{l}\text { Resampled training } \\
(\mathrm{NEC}=20 \%)\end{array}$ & 2.0 & 1.0 & 2.0 & 1.0 & 99.74 \\
\hline $\begin{array}{l}\text { SNAPPE training } \\
(\mathrm{NEC}=20 \%)\end{array}$ & 2.0 & 1.0 & 2.0 & 1.0 & 99.58 \\
\hline
\end{tabular}

Table 4.13: LIBSVM C-SVC results for the total population model

\begin{tabular}{lcccccccc}
\hline & TN & FP & TP & FN & $\begin{array}{c}\text { TOTAL } \\
\text { training }\end{array}$ & $\begin{array}{c}\text { SENSITIVITY } \\
\text { \% }\end{array}$ & $\begin{array}{c}\text { SPECIFICITY } \\
\%\end{array}$ & $\begin{array}{c}\text { CR } \\
\%\end{array}$ \\
\hline 27 variables & 4196 & 64 & 26 & 74 & 4360 & 26.00 & 98.50 & 96.83 \\
26 variables & 4190 & 70 & 26 & 74 & 4360 & 26.00 & 98.36 & 96.70 \\
25 variables & 4194 & 66 & 28 & 72 & 4360 & 28.00 & 98.45 & 96.83 \\
24 variables & 4197 & 63 & 28 & 72 & 4360 & 28.00 & 98.52 & 96.90 \\
23 variables & 4197 & 63 & 28 & 72 & 4360 & 28.00 & 98.52 & 96.90 \\
22 variables & 4207 & 53 & 29 & 71 & 4360 & 29.00 & 98.76 & 97.16 \\
21 variables & 4208 & 52 & 29 & 71 & 4360 & 29.00 & 98.78 & 97.18 \\
20 variables & 4207 & 53 & 30 & 70 & 4360 & 30.00 & 98.76 & 97.18 \\
19 variables & 4202 & 58 & 31 & 69 & 4360 & 31.00 & 98.64 & 97.09 \\
18 variables & 4202 & 58 & 31 & 69 & 4360 & 31.00 & 98.64 & 97.09 \\
17 variables & $\mathbf{4 2 0 1}$ & $\mathbf{5 9}$ & $\mathbf{3 2}$ & $\mathbf{6 8}$ & $\mathbf{4 3 6 0}$ & $\mathbf{3 2 . 0 0}$ & $\mathbf{9 8 . 6 2}$ & $\mathbf{9 7 . 0 9}$ \\
16 variables & 4202 & 58 & $\mathbf{3 1}$ & 69 & 4360 & 31.00 & $\mathbf{9 8 . 6 4}$ & $\mathbf{9 7 . 0 9}$ \\
\hline
\end{tabular}


Table 4.14: LIBSVM C-SVC results for the total population model

\begin{tabular}{|c|c|c|c|c|c|}
\hline 27 variables & 26 variables & 25 variables & 24 variables & 23 variables & 22 variables \\
\hline hrespr & lhema & lhema & lhema & lhema & hitnrat \\
\hline hsodium & Iglue & lgluc & lgluc & hitnrat & apnea \\
\hline SEX & hitnrat & hitnrat & hitnrat & lgluc & lgluc \\
\hline bthwt & hrespr & hrespr & hsodium & apnea & SEX \\
\hline hitnrat & apnea & apnea & hrespr & GestAge & lhema \\
\hline Po2fio2r & SEX & SEX & apnea & hsodium & GestAge \\
\hline LWB & lbloodp & lbloodp & lbloodp & SEX & Po2fio $2 r$ \\
\hline Iserum & Iserum & Iserum & SEX & bthwt & hgluc \\
\hline OI & lheartr & $\mathrm{OI}$ & LWB & Po2fio2r & LWB \\
\hline LANC & $\mathrm{OI}$ & hheartr & lheartr & hpco2 & bthwt \\
\hline hheartr & hheartr & hpco2 & hheartr & hgluc & lheartr \\
\hline Igluc & Po2fio2r & Po2fio2r & lurine & lserum & lbloodp \\
\hline lhema & ltempf & lurine & lserum & lurine & lsodium \\
\hline lbloodp & hpco2 & lheartr & lplt & LWBC & hpco2 \\
\hline lurine & hgluc & lsodium & OI & lplt & hsodium \\
\hline Isodium & LWB & hsodium & ltempf & lsodium & lplt \\
\hline Iplt & lurine & ltempf & hpco2 & 1po2po2 & OI \\
\hline hgluc & 1po2po2 & hgluc & Po2fio $2 r$ & hheart & 1po2po2 \\
\hline lheartr & Apgar5 & LWB & lsodium & OI & hheartr \\
\hline seizure & hsodium & lplt & hgluc & lheartr & lserum \\
\hline GestAge & lplt & lpo2po2 & 1po2po2 & ltempf & lurine \\
\hline ltempf & lsodium & bthwt & bthwt & lbloodp & ltempf \\
\hline apnea & bthwt & GestAge & GestAge & hrespr & \\
\hline hpco2 & GestAge & Apgar 5 & Apgar 5 & & \\
\hline Apgar5 & LANC & $L A N C$ & & & \\
\hline 1po2po2 & seizure & & & & \\
\hline hhema & & & & & \\
\hline
\end{tabular}

Table 4.14 continued

\begin{tabular}{llllll}
\hline 21 variables & 20 variables & 19 variables & 18 variables & 17 variables & 16 variables \\
\hline apnea & hitnrat & hitnrat & hitnrat & hitnrat & lhema \\
SEX & lhema & apnea & lgluc & lhema & hitnrat \\
lhema & lgluc & lgluc & apnea & Lgluc & lgluc \\
hitnrat & apnea & lhema & lhema & apnea & apnea \\
lgluc & SEX & Po2fio2r & SEX & SEX & lheartr \\
GestAge & GestAge & SEX & lheartr & lheartr & Po2fio2r \\
hpco2 & lheartr & lheartr & Po2fio2r & Po2fio2r & hheartr \\
po2fio2r & Po2fio2r & GestAge & hheartr & hheartr & GestAge \\
lheartr & bthwt & hheartr & GestAge & GestAge & hsodium \\
hgluc & lsodium & hgluc & lpo2po2 & hsodium & SEX \\
bthwt & hpco2 & bthwt & hsodium & lpo2po2 & lpo2po2 \\
lbloodp & hgluc & lsodium & hgluc & hpco2 & bthwt \\
lplt & lurine & hpco2 & lsodium & bthwt & hpco2 \\
hsodium & hsodium & lurine & bthwt & hgluc & hgluc \\
lsodium & lpo2po2 & lpo2po2 & lurine & lsodium & lsodium \\
lpo2po2 & lplt & hsodium & OI & lurine & lurine \\
LWBC & LWB & OI & hpco2 & OI & \\
OI & OI & lplt & lplt & & \\
hheartr & hheartr & $L W B$ & & & \\
lurine & $l b l o o d p$ & & & & \\
$l s e r u m$ & & & & & \\
\hline
\end{tabular}


The variables that dominated the LIBSVM C-SVC data sets were lhema, hitnrat and lgluc. Sensitivity analysis performed showed that their removal from the data set caused the most degradation in performance with respect to sensitivity. The best performance was observed with a 17 variable data set which gave a sensitivity of $32.00 \%$, specificity of $98.63 \%$ and classification rate of $97.09 \%$.

\subsection{Model Comparison: ANN vs. SVM}

The best performance with respect to verification results was found with the ANN RFW tool minimum data set (20 variables) from Model B. Sensitivity was $67.00 \%$, specificity $86.74 \%$ and classification rate $86.28 \%$. For the same population, the best

SVM results for sensitivity, specificity and classification rate were $32.00 \%, 98.63 \%$, and 97.09\% respectively. The ANN RFW tool obtains a higher sensitivity at the expense of a lower of specificity. However, in case of NEC this might be more appropriate as the complication has devastating consequences if it is not diagnosed in due time. It was also very interesting to note that although the number of NEC cases was fairly similar between all three models examined by the ANN RFW tool, especially for VLBW and GestAge $<33$ weeks, different minimal datasets were obtained.

The VLBW and GestAge $<33$ weeks models could not be compared between the ANN RFW and LIBSVM C-SVC tool as the models were not obtainable by SVM. This could be attributed to several factors which need further investigation:

1. The small number of minority cases (NEC) might not be enough to train the SVM properly due to the fact that the cases are not similar in nature. 
Ensuring that sufficient number of cases is kept for validation of training, potentially robs the network of additional information. Although this holds true for both ANN RFW and LIBSVM C-SVC, it was more of a problem for the later tool. ANN RFW performance did degrade for both the VLBW and GestAge $<33$ weeks models, compared to performance achieved by the total database model.

2. It is also very possible that babies making up the VLBW and GestAge $<33$ weeks populations are similar with respect to normal and NEC babies in those two populations of interest. Both of the classes might be exhibiting similar symptoms, making it hard for either tool to extract information about them and hence classify NEC correctly. This could explain why some of the variables deemed as 'independent' for the outcome of NEC using Spearman correlation (lurine, lgluc, lsodium, hpco2, SEX) are still present in the minimum data sets.

Lastly, the use of LIBSVM C-SVC needs to be validated for additional medical outcomes, ex. mortality, before conclusive observations can be made about the tool performance. The nature of NEC in general, from disease complexity to the small number of cases, makes it difficult to model and analyze. This results in misclassification of minority cases and was seen in the performance of the LIBSVM C-SVC tool.

\subsubsection{Additional ANN \& SVM models}

SNAPPE-II models contain only 9 input variables. Both the ANN RFW and LIBSVM C-SVC tools attempted to use just these variables to model NEC in the three populations of interest. The results of the findings are summarized in table 4.15. 
Table 4.15: SNAPPE-II performance of the ANN RFW and LIBSVM C-SVC tools

\begin{tabular}{llccc}
\hline & & $\begin{array}{c}\text { SENSITIVITY } \\
(\mathbf{\%})\end{array}$ & $\begin{array}{c}\text { SPECIFICITY } \\
(\boldsymbol{\%})\end{array}$ & $\begin{array}{c}\text { CR } \\
(\%)\end{array}$ \\
\hline ANN RFW & Total data & 64.00 & 66.85 & 66.78 \\
& VLBW & 59.80 & 54.85 & 55.22 \\
& GestAge $<33$ wks & $\mathrm{n} / \mathrm{a}^{*}$ & $\mathrm{n} / \mathrm{a}^{*}$ & $\mathrm{n} / \mathrm{a}^{*}$ \\
\hline \multirow{2}{*}{ LIBSVM C-SVC } & Total data & 6.00 & 98.94 & 94.63 \\
& VLBW & 4.44 & 99.66 & 94.17 \\
& GestAge $<33$ wks & 5.00 & 98.59 & 93.27 \\
\hline
\end{tabular}

n/a* - model did not converge

Both ANN RFW and LIBSVM C-SVC performed poorly with only SNAPPE-II variables, compared to the results obtained when the full set of 27 variables was used. However, one thing was apparent: other than the $S G A$ SNAPPE-II variable which was removed from NEC modeling, all ANN RFW models had at least 5 out 8 SNAPPE-II variables present in their minimum data set (table 4.16). This suggests that although on their own SNAPPE-II variables are not sufficient to predict the outcome of NEC, some of them have an influence on the outcome. LIBSVM C-SVC model contained only 3 of the 8 SNAPPE-II variables.

Table 4.16: Occurrence of SNAPPE-II variables in minimum datasets

\begin{tabular}{|c|c|c|c|c|c|}
\hline \multirow[b]{2}{*}{$\begin{array}{l}\text { SNAPPE-II } \\
\text { variable }\end{array}$} & \multicolumn{4}{|c|}{ ANN RFW tool } & \multirow{2}{*}{$\begin{array}{c}\text { LIBSVM C-SVC } \\
\text { Total } \\
\text { population }\end{array}$} \\
\hline & $\begin{array}{c}\text { Total } \\
\text { population } \\
\text { Model A }\end{array}$ & $\begin{array}{c}\text { Total } \\
\text { population } \\
\text { Model B }\end{array}$ & $\begin{array}{l}\text { VLBW } \\
\text { model }\end{array}$ & $\begin{array}{c}\text { GestAge }<33 w k s \\
\text { model }\end{array}$ & \\
\hline lbloodp & $\mathrm{x}$ & $\sqrt{ }$ & $\mathrm{x}$ & $\sqrt{ }$ & $\mathrm{x}$ \\
\hline ltempf & $\sqrt{ }$ & $\sqrt{ }$ & $\sqrt{ }$ & $\sqrt{ }$ & $\mathrm{x}$ \\
\hline po2fio $2 r$ & $\sqrt{ }$ & $\sqrt{ }$ & $\mathrm{x}$ & $\sqrt{ }$ & $\sqrt{ }$ \\
\hline lserum & $\sqrt{ }$ & $\sqrt{ }$ & $\sqrt{ }$ & $\sqrt{ }$ & $\mathrm{x}$ \\
\hline seizure & $\sqrt{ }$ & $\sqrt{ }$ & $\mathrm{x}$ & $\mathbf{x}$ & $\mathrm{x}$ \\
\hline lurine & $\sqrt{ }$ & $\sqrt{ }$ & $\sqrt{ }$ & $\sqrt{ }$ & $\sqrt{ }$ \\
\hline bthwt & $\sqrt{ }$ & $\sqrt{ }$ & $\sqrt{ }$ & $\sqrt{ }$ & $\sqrt{ }$ \\
\hline Apgar5 & $\sqrt{ }$ & $\sqrt{ }$ & $\sqrt{ }$ & $\sqrt{ }$ & $\mathrm{x}$ \\
\hline SGA & $\mathrm{n} / \mathrm{a}$ & $\mathrm{n} / \mathrm{a}$ & $\mathrm{n} / \mathrm{a}$ & $\mathrm{n} / \mathrm{a}$ & $\mathrm{n} / \mathrm{a}$ \\
\hline
\end{tabular}




\section{CONCLUSION}

The pathology of NEC is extremely complicated compared to other medical outcomes studied by MIRG. This medical complication is multivariable and effects different populations of babies in different ways. In response to this, the goal of this research was to develop a day 1 model for NEC for the following three populations: total CNN database, VLBW babies, and those born at less than 33 weeks of gestation.

Model development was performed using MIRG's ANN RFW tool that has been validated on outcome predictions for morality, ventilation, length of stay, BPD and IVH. The structures that performed best in all three models were non-linear, but made up of different variable sets. In the minimum data sets for all of the NEC models, at least five out of the 8 SNAPPE-II variables were retained.

NEC was also modeled with a support vector machine tool. Modeling with the LIBSVM C-SVC v2.86 tool, using the radial basis function kernel did not provide valid results for the VLBW and gestation age less than 33 weeks models. Efforts to produce a minimum data set for these two populations proved futile as majority of NEC cases were misclassified. The total database model gave better results, but only after minority cases for the training set were resampled to $20 \%$.

\subsection{Summary of Contributions}

1. NEC modeling was performed for the first time with the ANN RFW tool that has to date been used by MIRG for mortality, ventilation, length of stay, BPD and IVH 
models of outcome prediction. Three different ANN RFW models were developed: total population, VLBW and gestation age less than 33 weeks.

2. From the three ANN RFW models, it was shown that NEC is governed by different variables in premature infants, compared to the total population.

3. While searching for the minimum data set, the ANN RFW tool did not select a single linear structure as the 'best performing' one for NEC. This proves that nonlinear models are better at modeling such a multivariable medical complication.

4. NEC modeling was attempted with an SVM tool, and it was found that the tool performed better when rare outcomes were resampled up to $20 \%$. This suggests that data resampling needs to be considered for other data mining systems and not just for ANNs.

5. Performance of SNAPPE-II variables was assessed for NEC outcome prediction. As literature review suggested, it was confirmed that scoring system variables (SNAPPE-II) can not be expected to perform well for an outcome for which they were not developed.

\subsection{Future Research}

\section{Additional NEC models and validation}

1. MIRG is currently involved in the process of obtaining the latest CNN database. The database of interest involves data from 24 tertiary NICU members of the CNN. The data collected between January $5^{\text {th }}, 2005$ and December $31^{\text {st }}, 2005$ includes 11,424 admissions (including transfers and re-admissions between NICUs) for a total of 11,004 patient admissions (Zhang, Lee, \& Wingert A., 2006). As 
occurrences of NEC are suspected to vary from centre to centre and from year to year, the $2005 \mathrm{CNN}$ database would be used to validate the findings of current research.

2. NEC is often diagnosed post day 3. However, this model was not examined in the current research due to the large number of missing values in the day 3 data of the 1996-1997 CNN database. The aim is to develop a day 3 NEC model using the 2005 CNN database.

\section{Support Vector Machines}

3. The SVM tool used in this study needs to be validated for additional outcome prediction, among which are mortality, length of stay and ventilation, before further conclusions can be drawn about its performance.

4. In order to improve the performance of the standard SVM tools, weight reduction techniques for SVM tools need to be analyzed. These should then be applied to the LIBSVM C-SVC tool used in this research, as the current tool does not support this.

\section{Diagnostic imaging for NEC}

5. NEC is currently diagnosed and confirmed through X-ray imaging. In response to the need of a safer alternative imaging modality, MIRG and the author began collaboration with neonatologist Dr. Erika Bariciak in studying the performance of medical infrared imaging for NEC. Medical infrared imaging has to date been used in clinical diagnosis for breast cancer, extra-cranial vessel disease, neuro-musculoskeletal disorders and vascular disease, among others. For NEC, research protocols 
and ethical clearance have been obtained for the NICUs at the Children's Hospital of Eastern Ontario, as well as the Ottawa General Hospital. Data collection began in May 2007 and is in progress. Future work in this area deals with:

i. Validating the use of clinical infrared imaging in NEC

ii. Extracting features from the infrared images and using them to enrich the medical files with information, with the hope of improving the prediction performance of the CDSS tools (ANN RFW tool in particular)

\section{Additional data mining tools}

6. MIRG has established collaboration with Dr. Julio Valdés from the National Research Council of Canada (NRC) Institute for Information Technology. Dr. Valdés conducts research in the field of artificial intelligence, data mining and pattern recognition. The aim is to apply Dr. Valdés' hybrid neural network model to the CNN database that MIRG uses. Dr. Valdés' tool will aid in data mining of multivariate heterogeneous databases, such as the CNN, as the network has the ability to tolerate missing values, and use imaging information as input variables. 


\section{REFERENCES}

Apgar, MD. V. (1953). A Proposal for a New Method of Evaluation of the Newborn Infant. Current Researches in Anesthesia and Analgesia , 32(4), 260-267.

Bariciak, Dr. E. (2006, December 8). Personal communication.

Beachy, P., \& Deacon, J. (1993). Core Curriculum for Neonatal Intensive Care Nursing. Philadelphia: Saunders.

Bell, M. J., Ternberg, J. L., Feigin, R. D., Keating, J. P., Marshall, R., Barton, L., \& Brotherton, T. (1978). Neonatal Necrotizing Enterocolitis. Therapeutic Decisions Based upon Clinical Staging. Annals of Surgery, 187(1), 1-7.

Berner, E. S. (1998). Clinical Decision Support Systems: Theory and Practice. New York: Springer.

Bishop, C. M. (1995). Neural networks for pattern recognition. Oxford: Clarendon Press.

Buonomo, C. (1999). The Radiology of Necrotizing Enterocolitis. Radiologic Clinics of North America, 37(6), 1187-1198.

Buskard, T.T. (1994). Estimating duration of ventilation, length of stay, and mortality in the intensive care unit using artificial neural networks. Unpublished master's thesis, Department of Electrical Engineering, University of New Brunswick, Fredericton, NB, Canada.

Center for Devices and Radiological Health (CDRH). (2008) Medical X-Rays [Web Page]. URL http://www.fda.gov/cdrh/radhealth/products/medicalxray.html [2008, April 11].

Chang, C., \& Lin, C. (2001) LIBSVM: a library for support vector machines [Web Page]. URL http://www.csie.ntu.edu.tw/ cjlin/libsvm [2008, April 30].

Chen, P., Lin, C., \& Scholkopf, B. (2005). A tutorial on v-support vector machines. Applied Stochastic Models in Business and Industry, 21, 111-136.

Cios, K. J., \& Moore, G. W. (2002). Uniqueness of medical data mining. Artificial Intelligence in Medicine, 26, 1-24.

Cortes, C., \& Vapnik, V. (1995). Support-Vector Networks. Machine Learning, 20, 273297.

Cullen, D. J., Civetta, J. M., Briggs B.A, \& Ferrara, L. C. (1974). Critical Care Medicine, $\underline{2}(2), 57-60$. 
Di Lorenzo, M. (2000). A role for nitric oxide in pathogenesis of necrotizing enterocolitis: discovery, evaluation and design of a novel prophylactic therapy. Unpublished doctoral dissertation, University of Ottawa, Department of Cellular and Molecular Medicine, Ottawa, ON, Canada.

Dorling, J. S., \& Field, D. J. (2008). Value and validity of neonatal disease severity scoring systems. Archives of Disease in Childhood Fetal and Neonatal Edition, 93, F80-F82.

Dorling, J. S., Field, D. J., \& Manktelow, B. (2005). Neonatal disease severity scoring systems. Archives of Disease in Childhood Fetal and Neonatal Edition, 90, F11F16.

Ennett, C. M., Frize, M., \& Shaw, R. E. (1999). Methodologies for predicting coronary surgery outcomes. Proceedings of the First Joint BMES/EMBS Conference, (2).

Ennett, C. M. (2003). Imputation of Missing Values by Integrating Artificial Neural Networks and Case-based Reasoning. Unpublished doctoral dissertation, Department of Systems and Computer Engineering, Carleton University, Ottawa, ON, Canada.

Ennett, C. M., \& Frize, M. (2003). Weight-elimination neural networks applied to coronary surgery mortality prediction. IEEE Transactions on Information Technology in Biomedicine, 7(2), 86-92.

Ennett, C. M., Frize, M., \& Walker, C. R. (2001). Influence of missing values on artificial neural network performance. Medinfo, 10 (1), 449-453.

Epelman, M., Daneman, A., Navarro, O. M., Morag, I., Moore, A. M., Kim, J. H., Faingold, R., Taylor, G., \& Gerstle, J. T. (2007). Necrotizing Enterocolitis: Review of State-of-the-Art Imaging Findings with Pathologic Correlation. Radiographics, 27(2), 285-305.

Fausett, L. (1994). Fundamentals of neural networks: architectures, algorithms, and applications. Englewood Cliffs, NJ: Prentice-Hall.

Fawcett, T. (2006). An introduction to ROC analysis. Pattern Recognition Letters 27, $\underline{27}(8), 861-874$.

Frize, M., Ennett, C., \& Hebert, P. (2001). Improving the efficiency and effectiveness of artificial neural networks as clinical decision-support systems. Medinfo, 574.

Frize, M., Ennett, C. M., \& Charette, E. (2000). Automated optimization of the performance of artificial neural networks to estimate medical outcomes. Proc. 3rd IEEE-ITAB Conference (Information Technology Applications in Biomedicine) and ITIS (International Telemedical Information Society), 168-73. 
Frize, M., Wang, L., Ennett, C. M., Nickerson, B., Solven, F. G., \& Stevenson, M. (1998). New advances and validation of knowledge management tools for critical care using classifier techniques. Proceeding of the AMIA Symposium, 553-558.

Garg, A. X., Adhikari, N. K. J., McDonald, H., Rosas-Arellano, M. P., Devereaux, P. J., Beyene, J., Sam, J., \& Haynes, R. B. (2005). Effects of Computerized Clinical Decision Support Systems on Practitioner Performance and Patient Outcomes. Journal of American Medical Association, 293(10), 1223-1238.

Goh, A. T. C. (1995). Back-propagation neural networks for modelling complex systems. Artificial Intelligence in Engineering, 9, 143-151.

Gray, J. E., Richardson, D. K., McCormick, M. C. , Workman-Daniels, K., \& Goldmann, D. A. (1992). Neonatal therapeutic intervention scoring systems: a therapy-based severity-of-illness index. Pediatrics, 90(4), 561-567.

Gupta, S. K., Burke, G., \& Herson, V. C. (1994). Necrotizing Enterocolitis: Laboratory Indicators of Surgical Disease. Journal of Pediatric Surgery, 29(11), 1472-1475.

Hallstrom, M., Koivisto, A.-M., Janas, M., \& Tammela, O. (2006). Laboratory parameters predictive of developing necrotizing entercolitis in infants born before 33 weeks of gestation. Journal of Pediatric Surgery, 41(4), 792-798.

Haykin, S. (1999). Neural networks: a comprehensive foundation. Upper Saddle River, N.J.: Prentice Hall.

Herry, C. L. (2008). Segmentation and Extraction of Regions of Interest for Automated Detection of Anomalies in Clinical Thermal Infrared Images. Unpublished doctoral dissertation, Department of Systems and Computer Engineering, Carleton University, Ottawa, ON, Canada.

Hertz, J., Krogh, A., \& Palmer, R. G. (1991). Introduction to the theory of neural computation. Redwood City, CA: Addison-Wesley Publishing Company.

Kessler, U., Mungnirandr, A., Nelle, M., Nimmo, A. F., Zachariou, Z., \& Berger, S. (2006). A simple presurgical necrotizing enterocolitis-mortality scoring system. Journal of Perinatology, 26(12), 764-768.

Khan, S. (2006). A Comparison of Mortality Prediction Models for Neonatal Intensive Care Patients Using Artificial Neural Networks and Case-Based Reasoning. Unpublished doctoral dissertation, Department of Systems and Computer Engineering, Ottawa, ON, Canada.

Kling, P. J., \& Hutter, J. J. (2003). Hematologic Abnormalities in Severe Neonatal Necrotizing Enterocolitis: 25 Years Later. Journal of Perinatology, 23(7), 523530 . 
Klosgen, W., \& Zytkow, J. M. (2002). Handbook of data mining and knowledge discovery. Oxford, New York: Oxford University Press.

Kurgan, L. A., Cios, K. J., Tadeusiewicz R., Ogiela, M., \& Goodenday, L. S. (2001). Knowledge discovery approach to automated cardiac SPECT diagnosis. Artificial Intelligence in Medicine, 23(2), 149-169.

Larose, D. T. (2005). Discovering knowledge in data mining: an introduction to data mining. Hoboken, N.J. Wiley-Interscience.

Lin, P. W., \& Stoll, B. J. (2006). Necrotising Enterocolitis. The Lancet, 368(9543), 1271-1283.

Livingstone, D. J., \& Manallack, D. T. (1993). Statistics using neural networks: chance effects. Journal of Medicinal Chemistry, 36, 1295-1297.

Luig, M., \& Lui, K. (2005a). Epidemiology of necrotizing enterocolitis - Part I: Changing regional trends in extremely preterm infants over 14 years. Journal of Paediatrics and Child Health, 41(4), 169-173.

Luig, M., \& Lui, K. (2005b). Epidemiology of necrotizing enterocolitis - Part II: Risks and susceptibility of premature infants during the surfactant era: A regional study. Journal of Paediatrics and Child Health, 41(4), 174-179.

Maimon, O., \& Rokach, L. (2005). The Data Mining and Knowledge Discovery Handbook. New York, NY: Springer Science+Business Media, Inc.

Martin, S. A., \& Simone, S. (2006). Gastrointestinal System: Necrotizing Enterocolitis. M. C. Slota (editor), Core Curriculum for Pediatric Critical Care Nursing (2nd ed., pp. 529-531). Edinburgh: Elsevier Saunders.

Martinez-Tallo, E., Claure, N., \& Bancalari, E. (1997). Necrotizing Enterocolitis in FullTerm or Near-Term Infants: Risk Factors. Biology of the Neonate. 71(5), 292298.

Masters, T. (1993). Practical Neural Network Recipes in C++. Boston: Academic Press, Inc.

McAlmon, K. R. (2004). Necrotizing enterocolitis. J. P. Cloherty, E. C. Eichenwald, \& A. R. Stark (editors), Manual of Neonatal Care (5th ed., pp. 643-650). Philadelphia, PA: Lippincott Williams \& Wilkins.

McMillan, D., Chan, H. W. P., \& Lee, S. K. (1997). Canadian NICU Network Report 1996-1997. (Report No. 1).

Mladenic, D., Lavrac, N., Bohanec, M., \& Moyle, S. (2003). Data Mining and Decision Support: Integration and Collaboration. Boston: Kluwer Academic Publishers. 
Olden, J., \& Jackson, D. (2002). Illuminating the "black box": a randomization approach for understanding variable contributions in artificial neural networks. Ecological Modelling, 154(1), 135-150.

Pan, A. (2002). MIRG ANN Guide. NSERC Summer Student Report.

Panigrahi, P. (2006). Necrotizing Entrocolitis: A Practical Guide to its Prevention and Management. Pediatric Drugs, 8(3 ), 151-165.

Parry, G., Tucker, J., \& Tarnow-Mordi, W. (2003). CRIB II: an update of the clinical risk index for babies score. The Lancet, 361(9371), 1789-1791.

Perreault, L., \& Metzger, J. (1999). A pragmatic framework for understanding clinicial decision support. Journal of Healthcare Information Management, 13(2), 5-21.

Richardson, D. K., Corcoran, J. D., Escobar, G. J., \& Lee, S. K. (2001). SNAP-II and SNAPPE-II: Simplified newborn illness severity and mortality risk scores. Journal of Pediatrics, 138(1), 92-100.

Richardson, D. K., Gray, J. E., McCormick, M. C. , Workman, K., \& Goldmann, D. A. (1993). Score for Neonatal Acute Physiology: A Physiologic Severity Index for Neonatal Intensive Care. Pediatrics, 91(3), 617-623.

Russell, I. (1993). Neural Networks: Theory and Applications. The Journal of Undergraduate Mathematics and Its Applications, 14(1).

Rybchynski, D. (2005). Design of an Artificial Neural Network Research Framework to Enhance the Development of Clinical Prediction Models. Unpublished master's dissertation, School of Information Technology and Engineering, University of Ottawa, Ottawa, ON, Canada.

Sacha, J. P., Cios, K. J., \& Goodenday, L. S. (2000). Issues in automating cardiac SPECT diagnosis. IEEE Engineering in Medicine and Biology Magazine, 19(4), 78-88.

Scales, N. (2001). Mortality Prediction in an ICU and Coronary Surgery Database Using Neural Networks. ELG5196 Term Project. Ottawa, ON. School of Information Technology and Engineering, University of Ottawa.

Schölkopf, B., \& Smola, A. J. (2002). Learning with kernels: support vector machines, regularization, optimization, and beyond. Cambridge, MA: MIT Press.

Siedband, M. P. (1998). Medical Imaging Systems. J. G. Webster (editor), Medical Instrumentation: Application and Design (3rd ed., pp. 518-576). New York, NY: John Wiley \& Sons.

SPSS Inc. (2007). SPSS 16.0 for Windows (Version Release 16.0.1) Chicago: SPSS Inc. 
The International Neonatal Network. (1993). The CRIB (clinical risk index for babies) score: a tool for assessing initial neonatal risk and comparing performance of neonatal intensive care units. The Lancet, 342, 193-198.

Tong, Y. (2000). Developing ANN approaches to estimate neonatal ICU outcomes. Unpublished master's dissertation, School of Information Technology and Engineering, University of Ottawa, Ottawa, ON, Canada.

Townsend, D. (2007). Clinical trial of estimated risk stratification prediction tool. Unpublished master's dissertation, School of Information Technology and Engineering, University of Ottawa, Ottawa, ON, Canada.

Upperman, J. S., Camerini, V., Lugo, B., Yotov, I., Sullivan, J., Rubin, J., Clermont, G., Zamora, R., Ermentrout, G. B., Ford, H. R., \& Vodovotz, Y. (2007). Mathematical modeling in necrotizing enterocolitis - a new look at an ongoing problem. Journal of Pediatric Surgery, 42(3), 445-453.

Vapnik, V. N. (1995). The nature of statistical learning theory. New York, NY: Springer.

Vapnik, V. N. (1998). Statistical learning theory. New York, NY: Wiley.

Wang, L., \& Xiuju, F. (2005). Data mining with computational intelligence. Berlin, NY: Springer.

Weigend, A. S., Rumelhart, D. E., \& Huberman, B. A. (1990). Back-propagation, weightelimination and time series prediction. Proceedings of the 1990 Connectionist Models Summer School, 105-116.

Witten, I. H., \& Frank, E. (2005). Data Mining: Practical Machine Learning Tools and Techniques. San Francisco: Morgan Kaufmann.

Zhang, H. H., Liu, Y., Wu, Y., \& Zhu, J. (2008). Variable selection for multicategory SVM via adaptive supnorm regularization. Electronic Journal of Statistics, 2, 149167.

Zhang, X., Lee, S. K., \& Wingert A. (2006). CNN Annual Report 2005. (Report No. 5). 


\section{APPENDIX A: Features of NEC}

Table A-1: Clinical Characteristics and Laboratory \& Radiographic Features of NEC (McAlmon, 2004)

\begin{tabular}{|c|c|}
\hline Clinical Characteristics & Laboratory \& Radiographic Features \\
\hline $\begin{array}{l}\text { Systemic signs: } \\
\text { - } \quad \text { Respiratory distress } \\
\text { - } \quad \text { Apnea or bradycardia (or both) } \\
\text { - } \quad \text { Lethargy } \\
\text { - } \quad \text { Irritability } \\
\text { - } \quad \text { Poor feeding } \\
\text { - } \quad \text { Hypotension (low BP, shock) } \\
\text { - } \quad \text { Accidosis } \\
-\quad \text { Oliguria (reduced urine output) } \\
-\quad \text { Bleeding diathesis (hemorrhage) }\end{array}$ & $\begin{array}{l}\text { Radiographic features: } \\
\text { - } \quad \text { Dilated bowel loops } \\
\text { - } \quad \text { Abnormal gas pattern (consistent with ileus) } \\
\text { - } \quad \text { Pneumatosis intestinalis (gas within bowel } \\
\quad \text { wall; } \boldsymbol{K E Y} \text { radiographic trademark to } \\
\quad \text { confirm NEC diagnosis) } \\
\text { - } \quad \text { Portal / hepatic venous gas } \\
\text { - Pneumoperitoneum (air in abdominal cavity) }\end{array}$ \\
\hline $\begin{array}{l}\text { Abdominal signs: } \\
\text { - } \quad \text { Bloody stool (occult or gross blood) } \\
\text { - } \quad \text { Abdominal distension / tenderness } \\
\text { - } \quad \text { Gastric aspirates (feeding residuals) } \\
\text { - } \quad \text { Vomiting (blood, bile or both) } \\
\text { - } \quad \text { Abcreased / absent bowel sounds } \\
\text { inflammation), or induration (hardening } \\
\text { thickening of tissue) } \\
\text { - } \quad \text { Abdominal mass } \\
\text { - Ascites (fluid in abdomen) }\end{array}$ & $\begin{array}{l}\text { Blood studies: } \\
\text { - Thrombocytopenia (low platelet count) } \\
\text { - Persistent metabolic acidosis } \\
\text { - Severe refractory hyponatremia (abnormally } \\
\text { low concentration of sodium ( } \mathrm{Na}+\text { ) ions in } \\
\text { blood) }\end{array}$ \\
\hline $\begin{array}{l}\text { Slow and sudden presentation of abdor } \\
\text { istension, ileus (loss of bowel motility, pos } \\
\text { fection }\end{array}$ & $\begin{array}{l}\text { Analysis of stool: } \\
\text { - Presence of blood and carbohydrate } \\
\text { malabsorption indicate changes in intestinal } \\
\text { integrity } \\
\text { - Gross blood may be an indication of NEC } \\
\text { while occult can be present for other medical } \\
\text { reason }\end{array}$ \\
\hline
\end{tabular}


Table A-2: Modified Bell Staging Criteria (Bell et al., 1978), (Panigrahi, 2006)

\begin{tabular}{|c|c|}
\hline Stage & Findings \\
\hline Stage I A (NEC suspect) & $\begin{array}{l}\text { - Temperature instability, apnea, bradycardia, lethargy } \\
\text { - Abdominal distension, gastric residuals, poor feeding, bilious } \\
\text { vomiting, occult (hidden) blood in stool } \\
\text { - X-ray shows mild ileus }\end{array}$ \\
\hline Stage I B (NEC suspect) & - As for stage I A with gross (visible) blood in stool \\
\hline $\begin{array}{l}\text { Stage II A (definite NEC, } \\
\text { mildly ill) }\end{array}$ & $\begin{array}{l}\text { As for stage I B, with pneumatosis intestinalis, absent bowel sounds } \\
+/ \text { - abdominal tenderness }\end{array}$ \\
\hline $\begin{array}{l}\text { Stage II B (definite NEC, } \\
\text { moderately ill) }\end{array}$ & $\begin{array}{l}\text { - As for stage II A with metabolic acidosis, mild thrombocytopenia, } \\
\text { leukocytosis or leukopenia } \\
\text { - Abdominal cellulitis or right lower quadrant mass } \\
\text { - Portal venous gas, with or without ascites (peritoneal cavity fluid) }\end{array}$ \\
\hline $\begin{array}{l}\text { Stage III A (advanced NEC, } \\
\text { severely ill infant, bowel intact) }\end{array}$ & $\begin{array}{l}\text { As for stage II B with hemodynamic changes, hypotension, } \\
\text { respiratory acidosis, neutropenia, disseminated intravascular } \\
\text { coagulation } \\
\text { - Abdominal cellulitis or right lower quadrant mass }\end{array}$ \\
\hline $\begin{array}{l}\text { Stage III A (advanced NEC, } \\
\text { severely ill infant, perforated } \\
\text { bowel) }\end{array}$ & $\begin{array}{l}\text { - Same radiologic findings as in stage II B, with definite ascites } \\
\text { - Same as stage III A with ruptured bowel and pneumoperitoneum }\end{array}$ \\
\hline
\end{tabular}




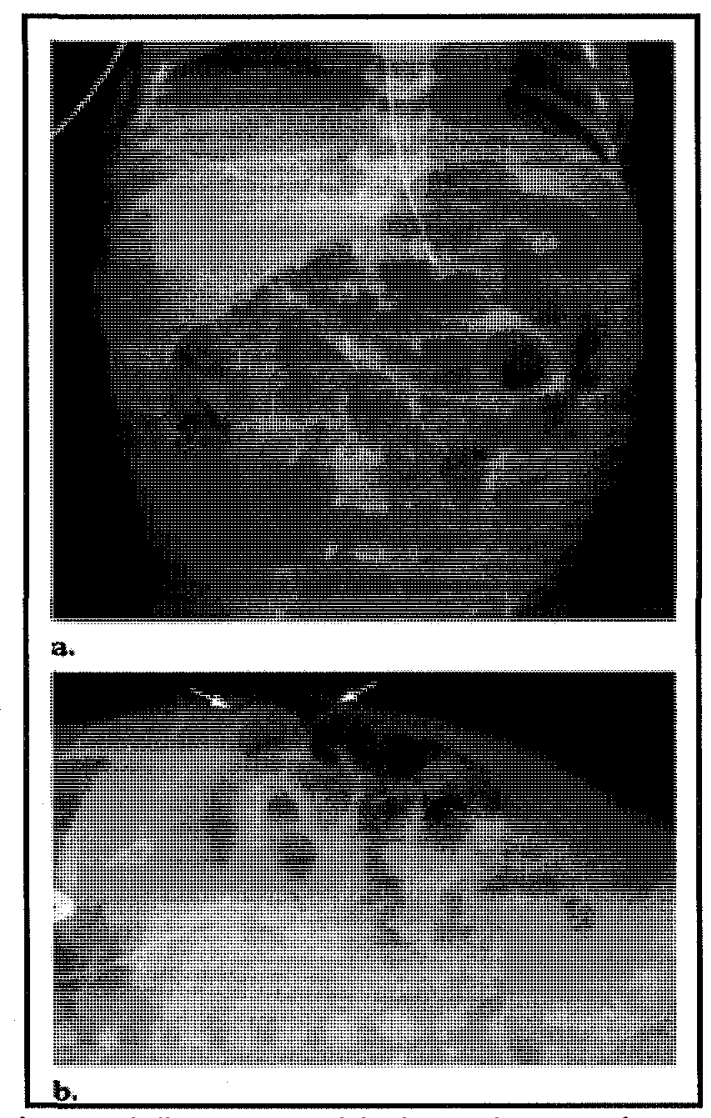

Figure A-1: (a) Supine and (b) cross-table lateral X-ray images of a NEC patient's abdomen (Epelman et al., 2007) 


\section{APPENDIX B: Neonatal Scoring Systems}

Table B-1: The Apgar Score (Beachy \& Deacon, 1993)

\begin{tabular}{lccc}
\hline & \multicolumn{3}{c}{ SCORE } \\
\hline COMPONENT & $\mathbf{0}$ & $\mathbf{1}$ & $\mathbf{2}$ \\
\hline Appearance (skin colour) & blue, pale & body pink, & pink \\
Pulse (heart rate) & absent & $<100 \mathrm{bmp}$ & $>100 \mathrm{bpm}$ \\
Grimace (reflex irritability) & no response & grimace & cough/sneeze/cry \\
Activity (muscle tone) & flaccid & some flexion & well flexed \\
Respiration (breathing) & absent & weak, irregular & good, crying \\
\hline
\end{tabular}

Table B-2: CRIB (The International Neonatal Network, 1993)

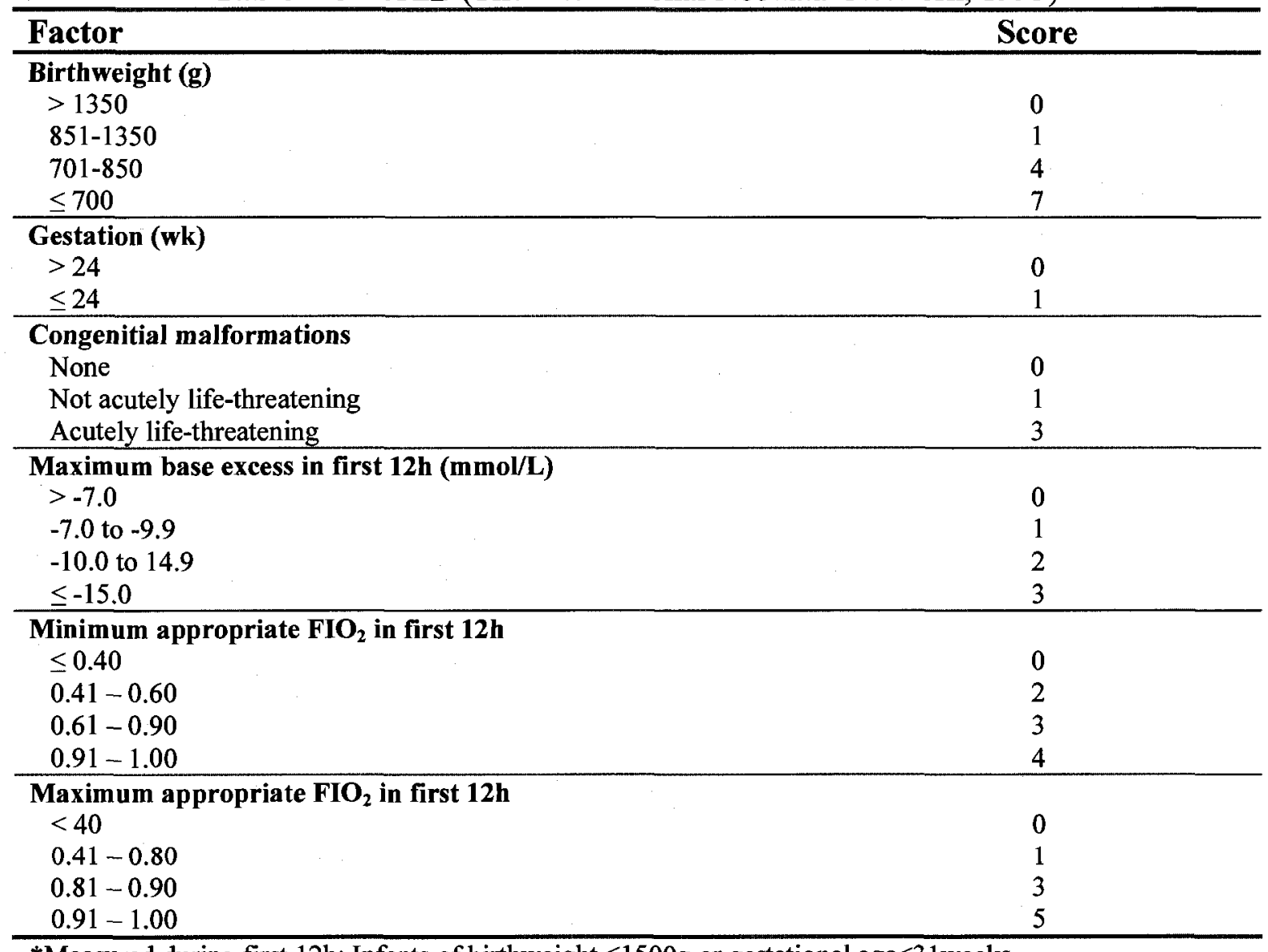

*Measured during first $12 \mathrm{~h}$; Infants of birthweight $\leq 1500 \mathrm{~g}$ or gestational age $<31$ weeks. 
Table B-3: CRIB II (Parry et al., 2003)

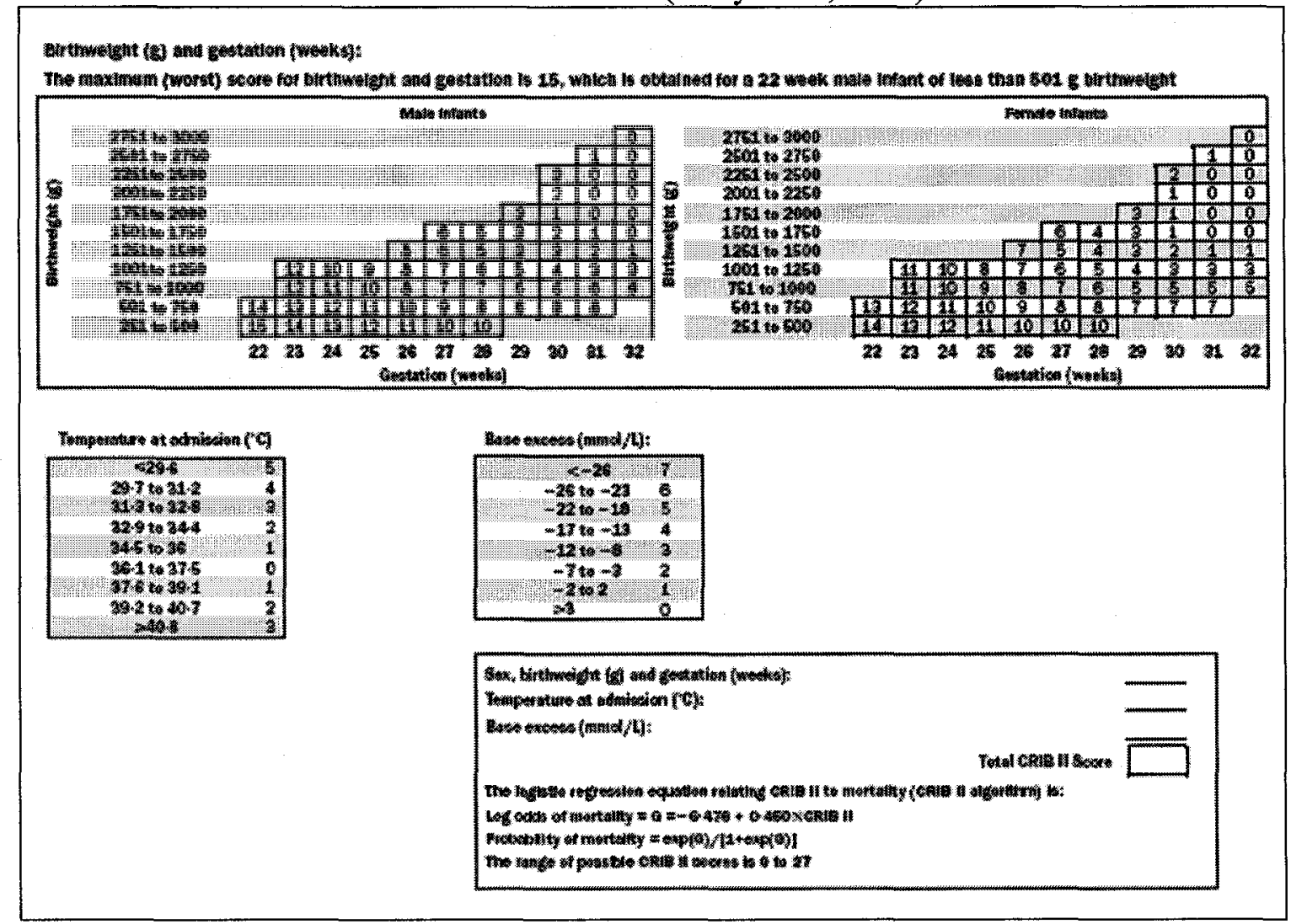


Table B-4: NTISS scoring system (Ennett, 2003)

\begin{tabular}{|c|c|c|c|}
\hline Item & Subscore & Item & Subscore \\
\hline RESPIRATORY & & MONITORING (con't) & \\
\hline Supplemental oxygen & $1^{\mathrm{a}}$ & Thermoregulated environment & 1 \\
\hline Surfactant administration & 1 & Noninvasive oxygen & 1 \\
\hline Tracheostomy care & $1^{b}$ & Arterial pressure & 1 \\
\hline Tracheostomy placement & $1^{b}$ & Central venous pressure & 1 \\
\hline Continuous positive airway pressure & $2^{a}$ & Urinary catheter & 1 \\
\hline administration & & Quantitative intake and output & 1 \\
\hline Endotracheal intubation & 2 & Extensive phlebotomy $(>10$ & $2^{h}$ \\
\hline Mechanical ventilation & $3^{\mathrm{a}}$ & blood draws) & \\
\hline Mechanical ventilation with muscle & $4^{a}$ & METABOLIC/NUTRITION & \\
\hline relaxation & & Gavage feeding & 1 \\
\hline High-frequency ventilation & $4^{\mathrm{a}}$ & Intravenous fat emulsion & 1 \\
\hline Extracorporeal membrane & 4 & Intravenous amino acid solution & 1 \\
\hline oxygenation & & Phototherapy & 1 \\
\hline CARDIOVASCULAR & & Insulin administration & 2 \\
\hline Indomethacin administration & 1 & Potassium infusion & 3 \\
\hline Volume expansion $(<=15 \mathrm{~mL} / \mathrm{kg})$ & $1^{\mathrm{c}}$ & TRANSFUSION & \\
\hline Vasopressor administration ( 1 agent) & $2^{d}$ & Intravenous gamma globulin & 1 \\
\hline Volume expansion (> $15 \mathrm{~mL} / \mathrm{kg})$ & $3^{\mathrm{c}}$ & Red blood cell $(<=15 \mathrm{~mL} / \mathrm{kg})$ & $2^{i}$ \\
\hline Vasopressor administration $(>1$ & $3^{\mathrm{d}}$ & Partial volume exchange & 2 \\
\hline agent) & & Red blood cell $(>15 \mathrm{~mL} / \mathrm{kg})$ & $3^{\mathrm{i}}$ \\
\hline Pacemaker on standby & $3^{2}$ & Platelet & 3 \\
\hline Pacemaker used & $4^{e}$ & White blood cell & 3 \\
\hline Cardiopulmonary resuscitation & 4 & Double volume exchange & 3 \\
\hline DRUG THERAPY & & PROCEDURAL & \\
\hline Antibiotic ( $<=2$ agents) & $1^{f}$ & Transport of patient & 2 \\
\hline Diuretic (enteral) & $1^{8}$ & Single chest tube in place & $2^{j}$ \\
\hline Steroid (postnatal) & 1 & Minor operation & $2^{k}$ \\
\hline Anticonvulsant & 1 & Multiple chest tubes in place & $3^{j}$ \\
\hline Aminophylline & 1 & Thoracentesis & 3 \\
\hline Other unscheduled medication & 1 & Major operation & $4^{k}$ \\
\hline Antibiotic ( $>2$ agents) & $2^{f}$ & Pericardiocentesis & $4^{1}$ \\
\hline Diuretic (parenteral) & $2^{\mathrm{g}}$ & Percardial tube in place & $4^{1}$ \\
\hline Treatment of metabolic acidosis & 3 & Dialysis & 4 \\
\hline Potassium binding resin & 3 & VASCULAR ACCESS & \\
\hline MONITORING & & Peripheral intravenous line & 1 \\
\hline Frequent vital signs & 1 & Arterial line & 2 \\
\hline Cardiorespiratory & 1 & Central venous line & 2 \\
\hline Phlebotomy (5-10 blood draws) & $1^{\mathrm{h}}$ & & \\
\hline
\end{tabular}

*Subscript letters represent mutually exclusive variables. 
Table B-5: SNAP scoring system (Richardson et al., 1993)

\begin{tabular}{|c|c|c|c|c|c|}
\hline \multirow[t]{2}{*}{ Variable } & & \multicolumn{4}{|c|}{ Point Range } \\
\hline & & 0 & 1 & 3 & 5 \\
\hline \multirow[t]{2}{*}{ Mean blood pressure, $\mathrm{mmHg}$} & High & $<66$ & $66-80$ & $81-100$ & $>100$ \\
\hline & Low & $>35$ & $30-35$ & $20-29$ & $<20$ \\
\hline \multirow[t]{2}{*}{ Heart rate } & High & $<180$ & $180-200$ & $201-250$ & $>250$ \\
\hline & Low & $>100$ & $80-100$ & $40-79$ & $<40$ \\
\hline Respiratory rate & High & $<60$ & $60-100$ & $>100$ & - \\
\hline Temperature $\left({ }^{\circ} \mathrm{F}\right)$ & Low & $>96$ & $95-96$ & $92-94.9$ & $<92$ \\
\hline $\mathrm{pO} 2, \mathrm{mmHg}$ & Low & $>65$ & $50-65$ & $30-50$ & $<30$ \\
\hline $\mathrm{pO} 2 / \mathrm{FiO} 2$ ratio & Low & $>3.5$ & $2.5-3.5$ & $0.3-2.49$ & $<0.3$ \\
\hline $\mathrm{pCO} 2, \mathrm{mmHg}$ & High & $<50$ & $50-65$ & $66-90$ & $>90$ \\
\hline Oxygenation index (OI) & High & $<0.07$ & $0.07-0.2$ & $0.21-0.40$ & $>0.40$ \\
\hline \multirow[t]{2}{*}{ Hematocrit, \% } & High & $<66$ & $66-70$ & $>70$ & - \\
\hline & Low & $>35$ & $30-35$ & $20-29$ & $<20$ \\
\hline White blood cell count $(\times 1000)$ & Low & $>5.0$ & $2.0-5.0$ & $<2.0$ & - \\
\hline Immature/total neutrophil ratio & High & $<0.21$ & $\geq 0.21$ & - & - \\
\hline Absolute neutrophil count & Low & $>999$ & $500-999$ & $<500$ & - \\
\hline Platelet count (x1000) & Low & $>100$ & $30-100$ & $0-29$ & - \\
\hline Blood urea nitrogen, mg/dL & High & $<40$ & $40-80$ & $>80$ & \\
\hline Creatinine, mg/dL & High & $<1.2$ & $1.2-2.4$ & $2.5-4.0$ & $>4.0$ \\
\hline Urine output, $\mathrm{mL} / \mathrm{kg} / \mathrm{h}$ & Low & $>0.9$ & $0.5-0.9$ & $0.1-0.49$ & $<0.1$ \\
\hline \multicolumn{6}{|l|}{ Indirect bilirubin (birth weight) } \\
\hline - >2kg: mg/dL & & $<15$ & $15-20$ & $>20$ & - \\
\hline$-\leq 2 \mathrm{~kg}: \mathrm{mg} / \mathrm{dL}$ & & $<5$ & $5-10$ & $>10$ & - \\
\hline Direct bilirubin, $\mathrm{mg} / \mathrm{dL}$ & & $<2.0$ & $>2.0$ & - & - \\
\hline \multirow[t]{2}{*}{ Sodium, $\mathrm{mEq} / \mathrm{L}$} & High & $<150$ & $150-160$ & $160-180$ & $>180$ \\
\hline & Low & $>130$ & $120-130$ & $<120$ & - \\
\hline \multirow[t]{2}{*}{ Potassium, $\mathrm{mEq} / \mathrm{L}$} & High & $<6.6$ & $6.6-7.5$ & $7.6-9.0$ & $>9.0$ \\
\hline & Low & $>2.9$ & $2.0-2.9$ & $<2.0$ & - \\
\hline \multirow[t]{2}{*}{ Calcium (ionized), $\mathrm{mg} / \mathrm{L}$} & High & $<1.4$ & $\geq 1.4$ & - & - \\
\hline & Low & $>1.0$ & $0.8-1.0$ & $<0.8$ & - \\
\hline \multirow[t]{2}{*}{ Calcium (total), $\mathrm{mg} / \mathrm{L}$} & High & $<12$ & $\geq 12$ & - & - \\
\hline & Low & $>6.9$ & $5.0-6.9$ & $<5.0$ & - \\
\hline \multirow[t]{2}{*}{ Glucose, mg/L } & High & $<150$ & $150-250$ & $>250$ & - \\
\hline & Low & $>40$ & $30-40$ & $<30$ & - \\
\hline \multirow[t]{2}{*}{ Serum bicarbonate, $\mathrm{mEq} / \mathrm{L}$} & High & $<33$ & $\geq 33$ & - & - \\
\hline & Low & $>15$ & $11-15$ & $\leq 10$ & - \\
\hline Serum $\mathrm{pH}$ & Low & $>7.30$ & $7.20-7.30$ & $7.10-7.19$ & $<7.10$ \\
\hline Presence of seizures & & None & Single & Multiple & - \\
\hline Presence of apnea & & None & $\begin{array}{l}\text { Responsive } \\
\text { to stimulation }\end{array}$ & $\begin{array}{l}\text { Unresponsive } \\
\text { to stimulation }\end{array}$ & $\begin{array}{l}\text { Complete } \\
\text { apnea }\end{array}$ \\
\hline Stool guaiac & & Negative & Positive & - & - \\
\hline
\end{tabular}

*Perinatal Extension (PE) variable points:

Birth weight

$\begin{array}{cl}\quad \leq 749 \mathrm{~g} & 30 \text { points } \\ 750-799 & 10 \text { points } \\ \text { Apgar }<7 \text { (at } 5 \mathrm{~min}) & 10 \text { points } \\ \text { SGA }\left(5^{\text {th }} \text { percentile }\right) & 5 \text { points }\end{array}$

Where assignment of points corresponds to the following:

Point 0: normal physiologic range/missing data

Point 1: physiologic deviation was sufficiently abnormal to merit careful monitoring

Point 3: deviation was such that most clinicians would alter therapy to correct it

Point 5: acutely life-threatening 
Table B-6: SNAPPE-II scoring system (Ennett, 2003)

\begin{tabular}{lll}
\hline Variable & Range & Points \\
\hline Lowest blood pressure & $\mathrm{MBP}^{\mathrm{a}} 20-29 \mathrm{mmHg}$ & 9 \\
& $\mathrm{MBP}^{\mathrm{a}}<20 \mathrm{mmHg}$ & 19 \\
Lowest temperature & $95-96^{\circ} \mathrm{F}$ & 8 \\
& $<95{ }^{\circ} \mathrm{F}$ & 15 \\
Lowest PO2/FiO2 ratio & $1.0-2.49$ & 5 \\
& $0.3-0.99$ & 16 \\
& $<0.3$ & 28 \\
Lowest serum pH & $7.10-7.19$ & 7 \\
& $<7.10$ & 16 \\
Multiple seizures & $>1$ & 19 \\
Lowest urine output & $0.1-0.9 \mathrm{~mL} / \mathrm{kg} / \mathrm{h}$ & 5 \\
& $<0.1 \mathrm{~mL} / \mathrm{kg} / \mathrm{h}$ & 18 \\
Birth weight & $750-999 \mathrm{~g}$ & 10 \\
& $<750 \mathrm{~g}$ & 17 \\
Small for gestational age & $<3^{\text {rd }} \mathrm{percentile}$ & 12 \\
Apgar score at 5 minutes & $<7$ & 18 \\
\hline
\end{tabular}

${ }^{* \mathrm{a}} \mathrm{MBP}=$ mean arterial blood pressure 